Supporting Information

\title{
An Organocatalytic Oxidative Coupling Strategy for the Direct Synthesis of Arylated Quaternary Stereogenic Centres
}

Katherine M. Bogle, David J. Hirst and Darren J. Dixon*

School of Chemistry, The University of Manchester, Oxford Road, Manchester, M13 9PL, UK

GlaxoSmithKline, New Frontiers Science Park, Third Avenue, Harlow, Essex, CM19 5AW, UK 


\section{Contents}

1. General Experimental 4

1.1 Solvents and reagents 4

1.2 Chromatography 4

1.3 Characterization 4

1.4 Starting materials $\quad 5$

2. Experimental Procedures 5

2.1 Quinone stability studies $\quad 5$

2.2 General procedure for Michael addition to o-quinones generated in-situ 6

2.3 Synthesis of enantioenriched adduct 11

$\begin{array}{ll}2.4 \text { Preparation of catalysts } & 15\end{array}$

$\begin{array}{ll}\text { 3. References } & 16\end{array}$

$\begin{array}{ll}\text { 4. Spectra } & 17\end{array}$

$\begin{array}{lll}4.1 & \text { Spectra for compound 8a } & 17\end{array}$

$\begin{array}{lll}4.2 & \text { Spectra for compound } \mathbf{8 b} & 19\end{array}$

$\begin{array}{lll}4.3 & \text { Spectra for compound } 8 \mathrm{c} & 21\end{array}$

$\begin{array}{lll}4.4 & \text { Spectra for compound 8d } & 23\end{array}$

$\begin{array}{lll}4.5 & \text { Spectra for compound } 8 \mathbf{e} & 25\end{array}$

$\begin{array}{lll}\text { 4.6 Spectra for compound } 8 \mathrm{f} & 27\end{array}$

$\begin{array}{lll}4.7 & \text { Spectra for compound } 8 \mathrm{~g} & 29\end{array}$

$\begin{array}{lll}4.8 & \text { Spectra for compound } \mathbf{8 h} & 31\end{array}$

$\begin{array}{lll}4.9 & \text { Spectra for compound } \mathbf{8} \mathbf{i} & 33\end{array}$

4.10 Spectra for compound 8j 
$\begin{array}{lll}4.12 & \text { Spectra for compound 8I } & 39\end{array}$

4.13 Spectra for compound 8m 41

4.14 Spectra for compound 8n 43

4.15 Spectra for compound 8o 45

$\begin{array}{lll}4.16 & \text { Spectra for compound 8p } & 47\end{array}$

4.17 Spectra for compound $11 \quad 49$

4.18 Spectra for catalyst $13 \quad 54$ 


\section{General experimental}

All reactions were performed with no effort to exclude air or moisture unless otherwise stated.

\subsection{Solvents and reagents}

Bulk solutions were evaporated under reduced pressure using a Büchi rotary evaporator. Reagents and solvents used were obtained from commercial suppliers and used without further purification. Petroleum ether refers to distilled light petroleum of fraction $\left(40-65^{\circ} \mathrm{C}\right)$.

Polymer-supported periodate $\left(\mathrm{ps}-\mathrm{IO}_{4}\right)$ was prepared from amberlite $\AA$ IRA-900 chloride form resin (Aldrich) as described by Hodge and co-workers. ${ }^{(1)}$ The periodate loading was determined through oxidation of an excess of 3,5-di-tert-butylcatechol (known to give a stable quinone) and calculation of the ratio of catechol:quinone products and was found to vary slightly between batches but is typically $4.3 \mathrm{mmol} / \mathrm{g}$. The resin obtained was ground using a pestle and mortar before use to allow more rapid washing of the reaction products from the resin. 2-tert-Butylimino-2-diethylamino-1,3-dimethylperhydro-1,3,2-diazaphosphorine, polymer-bound (polymer supported-BEMP or ps-BEMP) was purchased from Fluka and contains $2.2 \mathrm{mmol} / \mathrm{g}$ of base.

\subsection{Chromatography}

In all cases of chromatography, eluent solvents were used as supplied from commercial sources. Flash column chromatography was carried out using Merck Kiesegal 60 silica gel (230-400 mesh). Thin-layer chromatography (TLC) was carried out using Merck Kiesegal 60 $F_{254}$ (230-400 mesh) fluorescent treated silica which were visualised under UV light $(250 \mathrm{~nm})$ or by staining with aqueous potassium permanganate solutions as appropriate. Enantiomeric excesses were determined using high performance liquid chromatography (HPLC) performed on a Hewlett Packard 1050 series system (conditions given with compound).

\subsection{Characterization}

All ${ }^{1} \mathrm{H}$ and ${ }^{13} \mathrm{C}$ NMR spectra were recorded using a Bruker $500 \mathrm{MHz}$ and Varian $300 \mathrm{MHz}$ spectrometers and are using parts per million $(\mathrm{ppm})$ for measurement against a tetramethyl silane (TMS) internal standard. Chemical shifts $(\delta)$ are given in ppm, and coupling constants $(\mathrm{J})$ are given in Hertz $(\mathrm{Hz})$ to the nearest $0.5 \mathrm{~Hz}$. The ${ }^{1} \mathrm{H}$ NMR spectra are reported as follows: $\delta / p p m$ (number of protons, multiplicity, coupling constants $\mathrm{J} / \mathrm{Hz}$, and assignment). Multiplet abbreviations are as follows: $d$ doublet, $t$ triplet, $q$ quartet, $p$ quintet, $s$ sextet, $h$ heptet, $m$ multiplet, br broad. DEPT135 and two-dimensional (COSY, HMQC, HMBC) NMR spectroscopy was used where appropriate to assist the assignment of signals in the ${ }^{1} \mathrm{H}$ and

${ }^{13} \mathrm{C}$ NMR spectra. Low resolution mass spectrometry (electron impact/chemical ionization) was recorded on a Micromass Trio 2000 quadropole mass spectrometer and (electrospray) on a Micromass Platform II spectrometer. High resolution mass spectra (accurate mass) were 
recorded on a Thermo Finnigan Mat95XP mass spectrometer. Infrared spectra were recorded on a Perkin Elmer Spectrum RX1 FTIR spectrometer (thin film deposited onto a sodium chloride plate) and a Perkin Elmer spectrum BX FTIR spectrometer (solid), only selected absorbances $(\lambda \max )$ are reported. Optical rotations were recorded using an Optical Activity AA-1000 polarimeter; $[\alpha]_{D}$ values are reported in $10^{-1}$ degcm $^{2} \mathrm{~g}^{-1}$; concentration (c) is given in $\mathrm{g} / 100 \mathrm{ml}$ at $589 \mathrm{~nm}$. Melting points were recorded using a Gallenkamp melting point apparatus and are uncorrected.

\subsection{Starting materials}

1,2-naphthoquinone was purchased from Fluka and was used as supplied. All the 3substituted catechols used were prepared from the appropriate commercially available 2substituted phenols through a one pot formylation and Dakin oxidation procedure as described Hansen et. al. ${ }^{(2)}$

\section{Experimental Procedures}

\subsection{Quinone stability studies}

\subsubsection{General procedure for determining the decomposition rate of 3-substituted quinones by ${ }^{1} \mathrm{H}$ NMR}<smiles>[R]c1cccc(O)c1O</smiles>

1

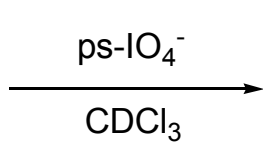

$\mathrm{CDCl}_{3}$<smiles>[R]C1=CC=CC(=O)C1=O</smiles>
decomposition

To a stirred solution of 3-substituted catechol (1) $(0.2 \mathrm{mmol})$ in $\mathrm{CDCl}_{3}(2 \mathrm{~mL})$ at $-20^{\circ} \mathrm{C}$ in a flask wrapped in tin foil was added polymer-supported periodate $\left(\mathrm{ps}-\mathrm{IO}_{4}\right)(0.4 \mathrm{mmol})$ and the resulting mixture stirred at this temperature for $20 \mathrm{~min}$. The cold solution was rapidly filtered directly into an NMR tube and an internal standard (methyl-3,4,5-trimethoxy benzoate) (approx. $10 \mathrm{mg}$ ) was added and NMR spectra recorded immediately (assumed to be $\mathrm{t}=0$ ) and at various time intervals over $24 \mathrm{~h}$. The distinctive o-quinone doublet at ca. $6-6.5 \mathrm{ppm}$ was integrated with respect to the internal standard and the data plotted using Microcal Origin software. Smooth curves were fitted to the data points (except for stable 1,2-naphthoquinone where a linear plot was appropriate) and approximate half-lives calculated as 8 hours for $3-$ methoxy-o-benzoquinone and 1 hour for 3-tert-butyl-o-benzoquinone. No decomposition of 1,2-naphthoquinone could be detected over the time period measured. 


\subsection{General procedure for Michael addition to quinones generated in-situ}

\subsubsection{Synthesis and characterization of compound 8a}<smiles>CC(C)(C)c1cccc(O)c1O</smiles>

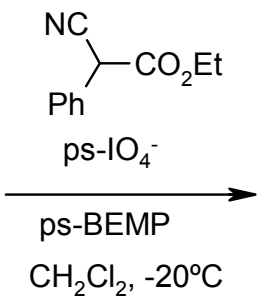<smiles>CCOC(=O)C(C)(C1=CC(=O)C(=O)C(C(C)(C)C)=C1)c1ccccc1</smiles><smiles>CCOC(=O)C(C)(c1cc(O)c(O)c(C(C)(C)C)c1)C(C)(C)c1ccccc1</smiles>

$8 a$

To a stirred mixture of 3-tert-butylcatechol $(166 \mathrm{mg}, 1.0 \mathrm{mmol}$ ), ethyl phenylcyanoacetate (189 mg, $1.0 \mathrm{mmol})$ and ps-BEMP (45 mg, $0.01 \mathrm{mmol})$ in $\mathrm{CH}_{2} \mathrm{Cl}_{2}(10 \mathrm{~mL})$ at $-20^{\circ} \mathrm{C}$, in a flask wrapped in foil, was added ps- $\mathrm{IO}_{4}(465 \mathrm{mg}, 2.0 \mathrm{mmol})$. The resulting suspension was stirred at this temperature for $30 \mathrm{~min}$ then acidified with acetic acid $(0.02 \mathrm{~mL})$ and filtered, washing the resin on the sinter with $\mathrm{CH}_{2} \mathrm{Cl}_{2}(5 \times 10 \mathrm{~mL})$ and THF $(1 \times 10 \mathrm{~mL})$. The deep red filtrate was concentrated to a volume of approximately $20 \mathrm{~mL}$ and stirred vigorously with a saturated aqueous solution of $\mathrm{Na}_{2} \mathrm{~S}_{2} \mathrm{O}_{4}(20 \mathrm{~mL})$ for $10 \mathrm{~min}$ to give a pale yellow solution. The organic layer was separated and the aqueous extracted with $\mathrm{CH}_{2} \mathrm{Cl}_{2}(2 \times 5 \mathrm{~mL})$. The combined organic portions were dried over sodium sulfate, filtered and concentrated by rotary evaporation. The residue was purified by chromatography on silica, eluting with petroleum ether containing $50-70 \%$ diethyl ether to give compound $\mathbf{8 a}(302 \mathrm{mg}, 85 \%$ ) as a cream solid; ${ }^{1} \mathrm{H}$ NMR $\left(500 \mathrm{MHz}, \mathrm{CDCl}_{3}\right) \delta_{\mathrm{H}} 7.38-7.33(5 \mathrm{H}, \mathrm{m}, 5$ of Ar-H), $6.86(1 \mathrm{H}, \mathrm{d}, \mathrm{J}=2.5 \mathrm{~Hz}, \mathrm{Ar}-\mathrm{H})$, $6.82(1 \mathrm{H}, \mathrm{d}, \mathrm{J}=2.5 \mathrm{~Hz}, \mathrm{Ar}-\mathrm{H}), 6.09(1 \mathrm{H}, \mathrm{s}, \mathrm{OH}), 5.89(1 \mathrm{H}, \mathrm{s}, \mathrm{OH}), 4.33(2 \mathrm{H}, \mathrm{dq}, \mathrm{J}=7.0,1.0$ $\left.\mathrm{Hz}, \mathrm{OCH}_{2} \mathrm{CH}_{3}\right), 1.36\left(9 \mathrm{H}, \mathrm{s}, \mathrm{C}\left(\mathrm{CH}_{3}\right)_{3}\right), 1.31\left(3 \mathrm{H}, \mathrm{t}, \mathrm{J}=7.0, \mathrm{OCH}_{2} \mathrm{CH}_{3}\right) ;{ }^{13} \mathrm{C}$ NMR $(125 \mathrm{MHz}$, $\left.\mathrm{CDCl}_{3}\right) \delta_{\mathrm{C}} 167.7,144.1,143,2,136.5,136.0,128.9,128.8,127.9,124.9,118.9,118.6,112.4$, 63.8, 58.6, 34.9, 29.2, 13.9; IR $v_{\max } / \mathrm{cm}^{-1} 3366,2257,1744,1433,1233,851$; MPT 167-169 ${ }^{\circ} \mathrm{C}$; MS $(\mathrm{Cl}+) \mathrm{m} / \mathrm{z}$ (relative intensity \%) $371\left(\mathrm{M}+\mathrm{NH}_{4}{ }^{+}, 100 \%\right)$, HRMS (ES+): calcd. for $\mathrm{C}_{21} \mathrm{H}_{27} \mathrm{O}_{4} \mathrm{~N}_{2}\left(\mathrm{M}+\mathrm{NH}_{4}{ }^{+}\right)$371.1965, found 371.1962.

\subsubsection{Synthesis and characterization of compound $8 b$}<smiles>CC(C)(C)c1cc(C2(C(=O)OC(C(F)(F)F)C(F)(F)F)CCCC2=O)cc(O)c1O</smiles>

According to method A, 3-tert-butylcatechol $(17 \mathrm{mg}, 0.1 \mathrm{mmol}$ ) was reacted with 1,1,1,3,3,3hexafluoropropan-2-yl 2-oxocyclopentanecarboxylate $(28 \mathrm{mg}, 0.1 \mathrm{mmol})$ to give, after $3 \mathrm{~h}$ 
reaction time, compound $\mathbf{8 b}(35 \mathrm{mg}, 79 \%)$ as an off-white solid after chromatography on silica, eluting with petroleum ether containing $50 \%$ diethyl ether; ${ }^{1} \mathbf{H}$ NMR $\left(500 \mathrm{MHz}, \mathrm{CDCl}_{3}\right)$ $\delta_{\mathrm{H}} 6.85(1 \mathrm{H}, \mathrm{d}, \mathrm{J}=2.0 \mathrm{~Hz}, \mathrm{Ar}-\mathrm{H}), 6.82(1 \mathrm{H}, \mathrm{d}, \mathrm{J}=2.0 \mathrm{~Hz}, \mathrm{Ar}-\mathrm{H}), 5.81(1 \mathrm{H}, \mathrm{s}, \mathrm{OH}), 5.79(1 \mathrm{H}, \mathrm{s}$, $\mathrm{OH}), 5.71\left(1 \mathrm{H}, \mathrm{h}, \mathrm{J}=6.0 \mathrm{~Hz} \mathrm{CH}\left(\mathrm{CF}_{3}\right)_{2}\right), 2.81-2.75\left(1 \mathrm{H}, \mathrm{m}, 1\right.$ of $\left.\mathrm{CH}_{2}\right), 2.72-2.67(1 \mathrm{H}, \mathrm{m}, 1$ of $\left.\mathrm{CH}_{2}\right), 2.53-2.40\left(2 \mathrm{H}, \mathrm{m}, \mathrm{CH}_{2}\right), 2.08-1.92\left(2 \mathrm{H}, \mathrm{m}, \mathrm{CH}_{2}\right), 1.38\left(9 \mathrm{H}, \mathrm{s}, \mathrm{C}\left(\mathrm{CH}_{3}\right)_{3} ;{ }^{13} \mathrm{C}\right.$ NMR $(75$ $\mathrm{MHz}, \mathrm{MeOD}) \delta_{\mathrm{C}} 211.9,169.7,146.2,145.6,137.4,124.8,123.9(\mathrm{~m}), 120.2(\mathrm{~m}), 118.1,112.7$, 68.1 (h, $\left.{ }^{2} J_{C, F} 34.5 \mathrm{~Hz}\right), 65.3,38.1,35.8,35.5,29.9,20.1$; IR $v_{\max } / \mathrm{cm}^{-1} 3488,2961,2535$, 1773, 1737; MPT 117-118 $\mathrm{C}$; MS (ES+) m/z (relative intensity \%) $460\left(\mathrm{M}+\mathrm{NH}_{4}{ }^{+}, 100 \%\right), 465$ $\left(\mathrm{M}+\mathrm{Na}^{+}, 85 \%\right)$, HRMS (El+): calcd. for $\mathrm{C}_{19} \mathrm{H}_{20} \mathrm{O}_{5} \mathrm{~F}_{6}\left(\mathrm{M}^{+}\right)$442.1209, found 442.1214.

\subsubsection{Synthesis and characterization of compound 8c}<smiles>COC(=O)C1(c2cc(O)c(O)c(C(C)(C)C)c2)CCCC1=O</smiles>

According to method A, 3-tert-butylcatechol ( $83 \mathrm{mg}, 0.5 \mathrm{mmol}$ ) was reacted with methyl 2oxocyclopentanecarboxylate $(142 \mathrm{mg}, 1.0 \mathrm{mmol}$ ) to give, after $4 \mathrm{~h}$ reaction time, compound $8 \mathrm{c}(119 \mathrm{mg}, 78 \%)$ as a cream solid after chromatography on silica, eluting with petroleum ether containing $50 \%$ diethyl ether; ${ }^{1} \mathbf{H}$ NMR $\left(500 \mathrm{MHz}, \mathrm{CDCl}_{3}\right) \delta_{\mathrm{H}} 6.79(1 \mathrm{H}, \mathrm{d}, \mathrm{J}=2.0, \mathrm{Ar}-\mathrm{H})$, $6.71(1 \mathrm{H}, \mathrm{d}, \mathrm{J}=2.0 \mathrm{~Hz}, \mathrm{Ar}-\mathrm{H}), 3.65\left(3 \mathrm{H}, \mathrm{s}, \mathrm{OCH}_{3}\right), 2.77-2.71(1 \mathrm{H}, \mathrm{td}, \mathrm{J}=13.5,6.5 \mathrm{~Hz}, 1$ of $\left.\mathrm{CH}_{2}\right), 2.52-2.46\left(1 \mathrm{H}, \mathrm{td}, \mathrm{J}=13.5,6.5 \mathrm{~Hz}, 1\right.$ of $\left.\mathrm{CH}_{2}\right), 2.44-2.25\left(2 \mathrm{H}, \mathrm{m}, \mathrm{CH}_{2}\right), 1.96-1.83(2 \mathrm{H}, \mathrm{m}$, $\left.\mathrm{CH}_{2}\right), 1.32\left(9 \mathrm{H}, \mathrm{s}, \mathrm{C}\left(\mathrm{CH}_{3}\right)_{3} ;{ }^{13} \mathrm{C}\right.$ NMR $\left(125 \mathrm{MHz}, \mathrm{CDCl}_{3}\right) \delta_{\mathrm{C}} 214.0,171.9,143.3,143.0,136.0$, 125.3, 117.2, 112.6, 64.6, 53.1, 37.8, 34.8, 34.8, 29.4, 19.3; IR $v_{\max } / \mathrm{cm}^{-1} 3261,1744,1715$, 1424, 1384, 1253; MPT $156-158^{\circ} \mathrm{C}$; MS $(\mathrm{Cl}+) \mathrm{m} / \mathrm{z}$ (relative intensity \%) $324\left(\mathrm{M}+\mathrm{NH}_{4}^{+}\right.$, $100 \%), 307\left(\mathrm{M}+\mathrm{H}^{+}, 20 \%\right)$, HRMS (ES+): calcd. for $\mathrm{C}_{17} \mathrm{H}_{26} \mathrm{O}_{5} \mathrm{~N}\left(\mathrm{M}+\mathrm{NH}_{4}{ }^{+}\right) 324.1805$, found 324.1805 .

\subsubsection{Synthesis and characterization of compound 8d}<smiles>COC(=O)C1(c2cc(O)c(O)c(C(C)(C)C)c2)Cc2ccccc2C1=O</smiles> 
According method A, 3-tert-butylcatechol (166 mg, $1.0 \mathrm{mmol}$ ) was reacted with methyl 1oxoindane-2-carboxylate $(190 \mathrm{mg}, 1.0 \mathrm{mmol}$ ) to give, after $3 \mathrm{~h}$ reaction time, compound $\mathbf{8 d}$ (299 $\mathrm{mg}, 84 \%$ ) as a pale orange solid after chromatography on silica, eluting with petroleum ether containing 50-70\% diethyl ether; ${ }^{1} \mathbf{H}$ NMR $\left(500 \mathrm{MHz}, \mathrm{CDCl}_{3}\right) \delta_{\mathrm{H}} 7.80(1 \mathrm{H}, \mathrm{d}, \mathrm{J}=7.5 \mathrm{~Hz}$, $\operatorname{Ar}-\mathrm{H}), 7.65(1 \mathrm{H}, \mathrm{dt}, \mathrm{J}=1.0,7.5 \mathrm{~Hz}, \operatorname{Ar}-\mathrm{H}), 7.50(1 \mathrm{H}, \mathrm{d}, \mathrm{J}=7.5 \mathrm{~Hz}, \operatorname{Ar}-\mathrm{H}), 7.40(1 \mathrm{H}, \mathrm{t}, \mathrm{J}=7.5$ $\mathrm{Hz}, \operatorname{Ar}-\mathrm{H}), 7.03(1 \mathrm{H}, \mathrm{d}, \mathrm{J}=2.5 \mathrm{~Hz}, \operatorname{Ar}-\mathrm{H}), 6.95(1 \mathrm{H}, \mathrm{s}, \mathrm{OH}), 6.77(1 \mathrm{H}, \mathrm{d}, \mathrm{J}=2.5 \mathrm{~Hz}, \mathrm{Ar}-\mathrm{H})$, $5.82(1 \mathrm{H}, \mathrm{s}, \mathrm{OH}), 4.20\left(1 \mathrm{H}, \mathrm{d}, \mathrm{J}=17.5 \mathrm{~Hz}, 1\right.$ of $\left.\mathrm{CH}_{2}\right), 3.71\left(3 \mathrm{H}, \mathrm{s}, \mathrm{O}-\mathrm{CH}_{3}\right), 3.61(1 \mathrm{H}, \mathrm{d}, \mathrm{J}=$ $17.5 \mathrm{~Hz}, 1$ of $\left.\mathrm{CH}_{2}\right), 1.37\left(9 \mathrm{H}, \mathrm{s}, \mathrm{C}\left(\mathrm{CH}_{3}\right)_{3}\right) ;{ }^{13} \mathrm{C}$ NMR $\left(125 \mathrm{MHz}, \mathrm{CDCl}_{3}\right) \delta_{\mathrm{C}} 202.1,171.5,152.6$, 143.2, 143.1, 136.1, 136.0, 134.8, 128.1, 128.0, 126.2, 125.2, 117.5, 112.1, 65.1, 53.4, 40.8, 34.8, 29.3; IR $v_{\max } / \mathrm{cm}^{-1} 3404,1700,1600,1425,1250,1213,959$; MPT 75-770 $\mathrm{C}$ MS (Cl+) $\mathrm{m} / \mathrm{z}$ (relative intensity \%) $372\left(\mathrm{M}+\mathrm{NH}_{4}{ }^{+}, 100 \%\right), 355\left(\mathrm{M}+\mathrm{H}^{+}, 50 \%\right)$; HRMS (ES+): calcd. for $\mathrm{C}_{21} \mathrm{H}_{23} \mathrm{O}_{5}\left(\mathrm{M}+\mathrm{H}^{+}\right)$355.1540, found 355.1543 .

\subsubsection{Synthesis and characterization of compound 8e}<smiles>COC(=O)C1(c2cc(O)c(O)c(-c3ccccc3)c2)Cc2ccccc2C1=O</smiles>

According to method A, 3-phenylcatechol (93 mg, $0.5 \mathrm{mmol}$ ) was reacted with methyl 1oxoindane-2-carboxylate $(190 \mathrm{mg}, 1.0 \mathrm{mmol}$ ) to give, after $5 \mathrm{~h}$ reaction time, compound $8 \mathrm{e}$ (145 $\mathrm{mg}, 77 \%$ ) as a pale yellow solid after chromatography on silica, eluting with petroleum ether containing 40-60\% diethyl ether; ${ }^{1} \mathrm{H}$ NMR $\left(500 \mathrm{MHz}, \mathrm{CDCl}_{3}\right) \delta_{\mathrm{H}} 7.82(1 \mathrm{H}, \mathrm{d}, \mathrm{J}=7.5 \mathrm{~Hz}$, Ar-H), $7.64(1 \mathrm{H}, \mathrm{dt}, \mathrm{J}=7.5,1.1 \mathrm{~Hz}, \mathrm{Ar}-\mathrm{H}), 7.49-7.35(7 \mathrm{H}, \mathrm{m}, \mathrm{Ar}-\mathrm{H}), 7.06(1 \mathrm{H}, \mathrm{d}, \mathrm{J}=2.5 \mathrm{~Hz}$, Ar-H), $6.85(1 \mathrm{H}, \mathrm{d}, \mathrm{J}=2.5 \mathrm{~Hz}, \mathrm{Ar}-\mathrm{H}), 4.19\left(1 \mathrm{H}, \mathrm{d}, \mathrm{J}=17.5 \mathrm{~Hz}, 1 \mathrm{H}\right.$ of $\left.\mathrm{CH}_{2}\right), 3.74(3 \mathrm{H}, \mathrm{s}$, $\left.\mathrm{OCH}_{3}\right), 3.62\left(1 \mathrm{H}, \mathrm{d}, \mathrm{J}=17.5 \mathrm{~Hz}, 1 \mathrm{H}\right.$ of $\left.\mathrm{CH}_{2}\right) ;{ }^{13} \mathrm{C} \mathrm{NMR}\left(125 \mathrm{MHz}, \mathrm{CDCl}_{3}\right) \delta_{\mathrm{C}} 200.8,171.2$, $152.2,144.1,140.1,136.8,135.8,134.8,130.5,129.0,128.9,128.2,128.0,127.8,126.2$, 125.2, 120.6, 113.9, 64.7, 53.4, 40.7; IR $v_{\max } / \mathrm{cm}^{-1} 3403,1702,1603,1430,1300,1251,1274$, 1212, 1178, 799, 754; MPT $164-166^{\circ} \mathrm{C}$; MS $(\mathrm{Cl}+) \mathrm{m} / \mathrm{z}$ (relative intensity \%) $392\left(\mathrm{M}+\mathrm{NH}_{4}{ }^{+}\right.$, $100 \%), 375\left(\mathrm{M}+\mathrm{H}^{+}, 20 \%\right)$; HRMS (ES+): calcd. for $\mathrm{C}_{23} \mathrm{H}_{22} \mathrm{O}_{5} \mathrm{~N}\left(\mathrm{M}+\mathrm{NH}_{4}{ }^{+}\right) 392.1492$, found 392.1491 . 


\subsubsection{Synthesis and characterization of compound $8 f$}<smiles>CCOC(=O)C(C#N)(c1ccccc1)c1cc(O)c(O)c(-c2ccccc2)c1</smiles>

According method A, 3-phenylcatechol $(61 \mathrm{mg}, 0.33 \mathrm{mmol})$ was reacted with ethyl phenylcyanoacetate $(125 \mathrm{mg}, 0.66 \mathrm{mmol}$ ) to give, after $4 \mathrm{~h}$ reaction time, compound $8 \mathrm{f}$ (89 $\mathrm{mg}, 72 \%)$ as a white solid after chromatography on silica, eluting with petroleum ether containing 40-60\% diethyl ether; ${ }^{1} \mathrm{H}$ NMR $\left(500 \mathrm{MHz}, \mathrm{CDCl}_{3}\right) \delta_{\mathrm{H}} 7.39-7.30(10 \mathrm{H}, \mathrm{m}, 10$ of ArH), 6.85-6.83 (2H, m, 2 of $\mathrm{Ar}-\mathrm{H}), 4.27\left(2 \mathrm{H}, \mathrm{q}, \mathrm{J}=7.0 \mathrm{~Hz}, \mathrm{OCH}_{2} \mathrm{CH}_{3}\right), 1.25(3 \mathrm{H}, \mathrm{t}, \mathrm{J}=7.0 \mathrm{~Hz}$, $\left.\mathrm{OCH}_{2} \mathrm{CH}_{3}\right) ;{ }^{13} \mathrm{C}$ NMR $\left(75 \mathrm{MHz}, \mathrm{CDCl}_{3}\right) \delta_{\mathrm{C}} 167.3,144.3,140.9,136.1,135.7,129.3,129.2$, 128.9, 128.9, 128.4, 128.2, 128.0, 127.9, 121.3, 118.8, 114.3, 63.7, 58.3, 13.9; IR $v_{\max } / \mathrm{cm}^{-1}$ $3438,3370,2360,2338,1737,1425,1308,1241,1006,852 ;$ MPT $126-130^{\circ} \mathrm{C} ; \mathrm{MS}(\mathrm{Cl}+) \mathrm{m} / \mathrm{z}$ (relative intensity \%) $391\left(\mathrm{M}+\mathrm{NH}_{4}{ }^{+}, 100 \%\right)$; HRMS (ES+): calcd. for $\mathrm{C}_{23} \mathrm{H}_{23} \mathrm{O}_{4} \mathrm{~N}_{2}\left(\mathrm{M}+\mathrm{NH}_{4}{ }^{+}\right)$ 391.1652, found 391.1652.

\subsubsection{Synthesis and characterization of compound $8 \mathrm{~g}$}<smiles>CCc1cc(C2(C(=O)OC)Cc3ccccc3C2=O)cc(O)c1O</smiles>

According to method A, 3-ethylcatechol $(138 \mathrm{mg}, 1.0 \mathrm{mmol}$ ) was reacted with methyl 1oxoindane-2-carboxylate $(190 \mathrm{mg}, 1.0 \mathrm{mmol}$ ) to give, after $6 \mathrm{~h}$ reaction time, compound $8 \mathrm{~g}$ (234 $\mathrm{mg}, 72 \%)$ as an orange oil after chromatography on silica, eluting with petroleum ether containing $50 \%$ diethyl ether; ${ }^{1} \mathrm{H}$ NMR $\left(500 \mathrm{MHz}, \mathrm{CDCl}_{3}\right) \delta_{\mathrm{H}} 7.79(1 \mathrm{H}, \mathrm{d}, \mathrm{J}=7.5 \mathrm{~Hz}, \mathrm{Ar}-\mathrm{H})$, $7.63(1 \mathrm{H}, \mathrm{dt}, \mathrm{J}=7.5,1.0 \mathrm{~Hz}, \mathrm{Ar}-\mathrm{H}), 7.47(1 \mathrm{H}, \mathrm{d}, \mathrm{J}=7.5 \mathrm{~Hz}, \mathrm{Ar}-\mathrm{H}), 7.36(1 \mathrm{H}, \mathrm{t}, \mathrm{J}=7.5 \mathrm{~Hz}, \mathrm{Ar}-$ H), $7.15(1 \mathrm{H}$, br. s, OH), $6.94(1 \mathrm{H}, \mathrm{d}, \mathrm{J}=2.5 \mathrm{~Hz}, \operatorname{Ar}-\mathrm{H}), 6.65(1 \mathrm{H}, \mathrm{d}, \mathrm{J}=2.5 \mathrm{~Hz}, \operatorname{Ar}-\mathrm{H}), 5.72$ $\left(1 \mathrm{H}\right.$, br. s, OH), $4.16\left(1 \mathrm{H}, \mathrm{d}, \mathrm{J}=17.5 \mathrm{~Hz}, 1 \mathrm{H}\right.$ of $\left.\mathrm{CH}_{2}\right), 3.70\left(3 \mathrm{H}, \mathrm{s}, \mathrm{OCH}_{3}\right), 3.59(1 \mathrm{H}, \mathrm{d}, \mathrm{J}=$ $17.5 \mathrm{~Hz}, 1 \mathrm{H}$ of $\left.\mathrm{CH}_{2}\right), 2.60\left(2 \mathrm{H}, \mathrm{q}, \mathrm{J}=7.5 \mathrm{~Hz}, \mathrm{CH}_{2} \mathrm{CH}_{3}\right), 1.17\left(3 \mathrm{H}, \mathrm{t}, \mathrm{J}=7.5 \mathrm{~Hz}, \mathrm{CH}_{2} \mathrm{CH}_{3}\right) ;{ }^{13} \mathrm{C}$ NMR $\left(125 \mathrm{MHz}, \mathrm{CDCl}_{3}\right) \delta_{\mathrm{C}} 201.7,171.5,152.5,143.1,142.0,135.9,134.7,130.7,129.2$, 128.0, 126.2, 125.2, 119.6, 112.1, 64.8, 53.4, 40.7, 23.1, 14.0; IR $v_{\max } / \mathrm{cm}^{-1} 3415,1700$, 1696, 1603, 1430, 1300, 1252, 1212, 1178, 940, 799; MS (Cl+) m/z (relative intensity \%) 344 $\left(\mathrm{M}+\mathrm{NH}_{4}{ }^{+}, 100 \%\right), 327\left(\mathrm{M}+\mathrm{H}^{+}, 50 \%\right)$; HRMS (ES+): calcd. for $\mathrm{C}_{19} \mathrm{H}_{19} \mathrm{O}_{5}\left(\mathrm{M}+\mathrm{H}^{+}\right) 327.1277$, found 327.1230. 


\subsubsection{Synthesis and characterization of compound $8 \mathrm{~h}$}<smiles>COC(=O)C1(c2cc(O)c(O)c(OC)c2)CCCC1=O</smiles>

According to method A, 3-methoxycatechol $(140 \mathrm{mg}, 1.0 \mathrm{mmol})$ was reacted with methyl 2oxocyclopentanecarboxylate $(142 \mathrm{mg}, 1.0 \mathrm{mmol})$ to give, after $30 \mathrm{~min}$ reaction time, compound $8 \mathrm{~h}$ (199 $\mathrm{mg}, 71 \%)$ as a pale brown solid after chromatography on silica, eluting with petroleum ether containing $30-50 \%$ diethyl ether; ${ }^{1} \mathrm{H}$ NMR $\left(500 \mathrm{MHz}, \mathrm{CDCl}_{3}\right) \delta_{\mathrm{H}} 6.61$ $(1 \mathrm{H}, \mathrm{d}, \mathrm{J}=2.0 \mathrm{~Hz}, \operatorname{Ar}-\mathrm{H}), 6.56(1 \mathrm{H}, \mathrm{d}, \mathrm{J}=2.0 \mathrm{~Hz}, \operatorname{Ar}-\mathrm{H}), 3.85\left(3 \mathrm{H}, \mathrm{s}, \mathrm{OCH}_{3}\right), 3.70(3 \mathrm{H}, \mathrm{s}$, $\left.\mathrm{OCH}_{3}\right),\left(1 \mathrm{H}, \mathrm{td}, \mathrm{J}=13.5,7.0 \mathrm{~Hz}, 1 \mathrm{H}\right.$ of $\left.\mathrm{CH}_{2}\right), 2.53-2.42\left(2 \mathrm{H}, \mathrm{m}, \mathrm{CH}_{2}\right), 2.39-2.32(1 \mathrm{H}, \mathrm{m}, 1$ of $\left.\mathrm{CH}_{2}\right), 2.01-1.86\left(2 \mathrm{H}, \mathrm{m}, \mathrm{CH}_{2}\right) ;{ }^{13} \mathrm{C}$ NMR $\left(125 \mathrm{MHz}, \mathrm{CDCl}_{3}\right) \delta_{\mathrm{C}} 212.3,171.4,146.8,143.7$, 132.1, 127.2, 107.9, 102.9, 64.3, 56.2, 53.0, 37.7, 34.9, 19.2; IR $\mathrm{U}_{\max } / \mathrm{cm}^{-1} 3436,1700,1601$, 1426, 1252, 960; MPT $114-116^{\circ} \mathrm{C}$; MS $(\mathrm{Cl}+) \mathrm{m} / \mathrm{z}$ (relative intensity \%) $298\left(\mathrm{M}+\mathrm{NH}_{4}{ }^{+}, 100 \%\right)$, $281\left(\mathrm{M}+\mathrm{H}^{+}, 25 \%\right)$; HRMS (ES+): calcd. for $\mathrm{C}_{14} \mathrm{H}_{20} \mathrm{O}_{6} \mathrm{~N}\left(\mathrm{M}+\mathrm{NH}_{4}{ }^{+}\right) 298.1285$, found 298.1283.

\subsubsection{Synthesis and characterization of compound $8 \mathrm{i}$}<smiles>COC(=O)C1(c2cc(O)c(O)c(OC)c2)Cc2ccccc2C1=O</smiles>

According to method A, 3-methoxycatechol $(140 \mathrm{mg}, 1.0 \mathrm{mmol})$ was reacted with methyl 1oxoindane-2-carboxylate $(190 \mathrm{mg}, 1.0 \mathrm{mmol}$ ) to give, after $2 \mathrm{~h}$ reaction time, compound $8 \mathbf{i}$ (289 $\mathrm{mg}, 88 \%$ ) as a viscous orange oil after chromatography on silica, eluting with diethyl ether; ${ }^{1} \mathrm{H}$ NMR $\left(500 \mathrm{MHz}, \mathrm{CDCl}_{3}\right) \delta_{\mathrm{H}} 7.82(1 \mathrm{H}, \mathrm{d}, \mathrm{J}=7.5 \mathrm{~Hz}, \mathrm{Ar}-\mathrm{H}), 7.64(1 \mathrm{H}, \mathrm{t}, \mathrm{J}=7.5 \mathrm{~Hz}$, ArH), $7.49(1 \mathrm{H}, \mathrm{d}, \mathrm{J}=7.5 \mathrm{~Hz}, \operatorname{Ar}-\mathrm{H}), 7.41(1 \mathrm{H}, \mathrm{t}, \mathrm{J}=7.5 \mathrm{~Hz}, \operatorname{Ar}-\mathrm{H}), 6.65(1 \mathrm{H}, \mathrm{d}, \mathrm{J}=2.0 \mathrm{~Hz}, \operatorname{Ar}-$ H), $6.59(1 \mathrm{H}, \mathrm{d}, \mathrm{J}=2.0 \mathrm{~Hz}, \mathrm{Ar}-\mathrm{H}), 5.35(1 \mathrm{H}, \mathrm{s}, \mathrm{OH}), 5.25(1 \mathrm{H}, \mathrm{s}, \mathrm{OH}), 4.18(1 \mathrm{H}, \mathrm{d}, \mathrm{J}=17.5$ $\mathrm{Hz}, 1 \mathrm{H}$ of $\left.\mathrm{CH}_{2}\right), 3.87\left(3 \mathrm{H}, \mathrm{s}, \mathrm{OCH}_{3}\right), 3.74\left(3 \mathrm{H}, \mathrm{s}, \mathrm{OCH}_{3}\right), 3.55\left(1 \mathrm{H}, \mathrm{d}, \mathrm{J}=17.5 \mathrm{~Hz}, 1 \mathrm{H}\right.$ of $\left.\mathrm{CH}_{2}\right)$; ${ }^{13} \mathrm{C} \mathrm{NMR}\left(125 \mathrm{MHz}, \mathrm{CDCl}_{3}\right) \delta_{\mathrm{C}} 200.2,171.1,152.1,146.8,143.7,135.7,134.9,131.9,130.1$, 128.0, 126.2, 125.1, 108.0, 102.7, 64.8, 56.2, 53.3, 40.8; IR $v_{\max } / \mathrm{cm}^{-1} 3415,1701,1604$, $1518,1251,1200,1176,1091,797,754 ; \mathrm{MS}(\mathrm{Cl}+) \mathrm{m} / \mathrm{z}$ (relative intensity \%) $346\left(\mathrm{M}+\mathrm{NH}_{4}{ }^{+}\right.$, 
$100 \%), 329\left(\mathrm{M}+\mathrm{H}^{+}, 60 \%\right)$; HRMS (ES+): calcd. for $\mathrm{C}_{18} \mathrm{H}_{17} \mathrm{O}_{6}\left(\mathrm{M}+\mathrm{H}^{+}\right) 329.1020$, found 329.1016 .

\subsubsection{Synthesis and characterization of compound 8j}<smiles>COC(=O)C1(c2cc(O)c(O)c(OC)c2)Cc2ccc(OC)cc2C1=O</smiles>

According to the above procedure 3-methoxycatechol $(54 \mathrm{mg}, 0.39 \mathrm{mmol})$ was reacted with methyl 6-methoxy-1-oxoindane-2-carboxylate $(56 \mathrm{mg}, 0.39 \mathrm{mmol}$ ) to give, after $2 \mathrm{~h}$ reaction time, compound $8 \mathbf{j}$ (117 $\mathrm{mg}, 84 \%$ ) as a cream solid after filtering through a small plug of silica, eluting with diethyl ether; ${ }^{1} \mathrm{H}$ NMR $\left(500 \mathrm{MHz}, \mathrm{CDCl}_{3}\right) \delta_{\mathrm{H}} 7.37(1 \mathrm{H}, \mathrm{d}, \mathrm{J}=9.0 \mathrm{~Hz}, \mathrm{Ar}-\mathrm{H})$, 7.24-7.22 (2H, m, 2 of Ar-H), $6.62(1 \mathrm{H}, \mathrm{d}, \mathrm{J}=2.0 \mathrm{~Hz}, \operatorname{Ar}-\mathrm{H}), 6.58(1 \mathrm{H}, \mathrm{d}, \mathrm{J}=2.0 \mathrm{~Hz}, \operatorname{Ar}-\mathrm{H})$, $5.39(1 \mathrm{H}, \mathrm{s}, \mathrm{OH}), 5.31(1 \mathrm{H}, \mathrm{s}, \mathrm{OH}), 4.08\left(1 \mathrm{H}, \mathrm{d}, \mathrm{J}=17.0 \mathrm{~Hz}, 1 \mathrm{H}\right.$ of $\left.\mathrm{CH}_{2}\right), 3.86\left(3 \mathrm{H}, \mathrm{s}, \mathrm{OCH}_{3}\right)$, $3.84\left(3 \mathrm{H}, \mathrm{s}, \mathrm{OCH}_{3}\right), 3.74\left(3 \mathrm{H}, \mathrm{s}, \mathrm{OCH}_{3}\right), 3.46\left(1 \mathrm{H}, \mathrm{d}, \mathrm{J}=17.0 \mathrm{~Hz}, 1 \mathrm{H}\right.$ of $\left.\mathrm{CH}_{2}\right) ;{ }^{13} \mathrm{C} \mathrm{NMR}^{(125}$ $\left.\mathrm{MHz}, \mathrm{CDCl}_{3}\right) \delta_{\mathrm{C}} 200.2,171.1,159.8,146.8,145.0,143.7,136.0,131.9,130.1,126.9,125.2$, 108.0, 106.0, 102.7, 65.6, 56.9, 55.6, 53.3, 40.2; IR $v_{\max } / \mathrm{cm}^{-1} 3444,3221,1705,1615,1517$, 1495, 1432, 1307, 1275, 1195, 1173, 1107, 1023, 815; MPT 143-145 $\mathrm{C}$; MS (Cl+) m/z (relative intensity \%) $359\left(\mathrm{M}+\mathrm{H}^{+}, 100 \%\right)$; HRMS (El+): calcd. for $\mathrm{C}_{19} \mathrm{H}_{18} \mathrm{O}_{7}\left(\mathrm{M}^{+}\right) 358.1047$, found 358.1052 .

\subsubsection{Synthesis and characterization of compound 8k}<smiles>CCCCOC(=O)C1(c2cc(O)c(O)c(OC)c2)Cc2cc(Br)ccc2C1=O</smiles>

According to the above procedure 3-methoxycatechol (14 $\mathrm{mg}, 0.1 \mathrm{mmol}$ ) was reacted with methyl 5-bromo-2,3-dihydro-1-oxo-1H-indene-2-carboxylate (31 $\mathrm{mg}, 0.1 \mathrm{mmol}$ ) to give, after 2 $\mathrm{h}$ reaction time, compound $\mathbf{8 k}(36 \mathrm{mg}, 80 \%)$ as a pale yellow oil after chromatography on silica, eluting with petroleum ether containing $50-70 \%$ diethyl ether; ${ }^{1} \mathbf{H} \mathbf{~ N M R}(500 \mathrm{MHz}$, $\left.\mathrm{CDCl}_{3}\right) \delta_{\mathrm{H}} 7.66(1 \mathrm{H}, \mathrm{s}, \mathrm{Ar}-\mathrm{H}), 7.65(1 \mathrm{H}, \mathrm{d}, \mathrm{J}=8.5 \mathrm{~Hz}, \mathrm{Ar}-\mathrm{H}), 7.54(1 \mathrm{H}, \mathrm{d}, \mathrm{J}=8.5 \mathrm{~Hz}, \operatorname{Ar}-\mathrm{H})$, $6.68(1 \mathrm{H}, \mathrm{d}, \mathrm{J}=2.0 \mathrm{~Hz}, \operatorname{Ar}-\mathrm{H}), 6.57(1 \mathrm{H}, \mathrm{d}, \mathrm{J}=2.0 \mathrm{~Hz}, \operatorname{Ar}-\mathrm{H}), 5.37(1 \mathrm{H}, \mathrm{s}, \mathrm{OH}), 5.28(1 \mathrm{H}, \mathrm{s}$, 
$\mathrm{OH}), 4.04\left(1 \mathrm{H}, \mathrm{d}, \mathrm{J}=17.5 \mathrm{~Hz}, 1 \mathrm{H}\right.$ of $\left.\mathrm{CH}_{2}\right), 3.87\left(3 \mathrm{H}, \mathrm{s}, \mathrm{OCH}_{3}\right), 3.52(1 \mathrm{H}, \mathrm{d}, \mathrm{J}=17.5 \mathrm{~Hz}, 1 \mathrm{H}$ of $\left.\mathrm{CH}_{2}\right), 1.39\left(9 \mathrm{H}, \mathrm{s}, \mathrm{C}\left(\mathrm{CH}_{3}\right)_{3}\right) ;{ }^{13} \mathrm{C} \mathrm{NMR}\left(75 \mathrm{MHz}, \mathrm{CDCl}_{3}\right) \delta_{\mathrm{C}} 199.3,169.0,153.5,146.7,143.6$, 134.0, 131.9, 131.5, 130.7, 129.8, 129.4, 126.1, 108.0, 102.9, 82.7, 65.5, 56.2, 40.0, 27.7; IR $v_{\max } / \mathrm{cm}^{-1} 3422,2976,2930,1735,1716 ;$ MS (ES+) $\mathrm{m} / \mathrm{z}$ (relative intensity \%) $471\left(\mathrm{M}+\mathrm{Na}^{+}\right.$, 100\%), $473\left(\mathrm{M}+\mathrm{Na}^{+}, 100 \%\right)$; HRMS (El+): calcd. for $\mathrm{C}_{21} \mathrm{H}_{25} \mathrm{O}_{6} \mathrm{NBr}\left(\mathrm{M}+\mathrm{NH}_{4}{ }^{+}\right)$466.0860, found 466.0863 .

\subsubsection{Synthesis and characterization of compound 8I}<smiles>COc1cc(C2(C(C)=O)CCCC2=O)cc(O)c1O</smiles>

According to method A, 3-methoxycatechol (140 mg, $1.0 \mathrm{mmol}$ ) was reacted with 2acetylcyclopentanone (126 mg, $1.0 \mathrm{mmol}$ ) to give, after $4 \mathrm{~h}$ reaction time, compound $8 \mathbf{8}$ (191 $\mathrm{mg}, 72 \%$ ) as a viscous yellow oil after chromatography on silica, eluting with petroleum ether containing 60-80\% diethyl ether; ${ }^{1} \mathrm{H}$ NMR $\left(500 \mathrm{MHz}, \mathrm{CDCl}_{3}\right) \delta_{\mathrm{H}} 6.54(1 \mathrm{H}, \mathrm{d}, \mathrm{J}=2.0 \mathrm{~Hz}, \mathrm{Ar}-\mathrm{H})$, $6.43(1 \mathrm{H}, \mathrm{d}, \mathrm{J}=2.0 \mathrm{~Hz}, \mathrm{Ar}-\mathrm{H}), 5.62(2 \mathrm{H}, \mathrm{br} . \mathrm{s}, 2 \mathrm{OH}), 3.85\left(3 \mathrm{H}, \mathrm{s}, \mathrm{OCH}_{3}\right), 2.86(1 \mathrm{H}, \mathrm{td}, \mathrm{J}=$ 13.5, $6.5 \mathrm{~Hz}, 1 \mathrm{H}$ of $\left.\mathrm{CH}_{2}\right), 2.38\left(2 \mathrm{H}, \mathrm{dt}, \mathrm{J}=7.5,3.5 \mathrm{~Hz}, \mathrm{CH}_{2}\right), 2.21(1 \mathrm{H}, \mathrm{td}, \mathrm{J}=13.5,6.5 \mathrm{~Hz}, 1$ of $\left.\mathrm{CH}_{2}\right), 2.07\left(3 \mathrm{H}, \mathrm{s}, \mathrm{CO}-\mathrm{CH}_{3}\right), 1.90-1.81\left(2 \mathrm{H}, \mathrm{m}, \mathrm{CH}_{2}\right) ;{ }^{13} \mathrm{C}$ NMR $\left(75 \mathrm{MHz}, \mathrm{CDCl}_{3}\right) \delta_{\mathrm{C}} 213.9$, 204.2, 147.2, 144.1, 132.1, 129.5, 107.6, 102.0, 71.9, 56.3, 39.2, 34.2, 26.6, 19.0; IR $v_{\max } / \mathrm{cm}^{-}$ ${ }^{1} 3426,1737,1729,1698,1613,1517,1204,1091 ; \mathrm{MS}(\mathrm{Cl}+) \mathrm{m} / \mathrm{z}$ (relative intensity \%) $282(\mathrm{M}$ $\left.+\mathrm{NH}_{4}{ }^{+}, 100 \%\right), 265\left(\mathrm{M}+\mathrm{H}^{+}, 20 \%\right)$; HRMS (ES+): calcd. for $\mathrm{C}_{14} \mathrm{H}_{20} \mathrm{O}_{5} \mathrm{~N}\left(\mathrm{M}+\mathrm{NH}_{4}^{+}\right) 282.1336$, 282.1335 .

\subsubsection{Synthesis and characterization of compound $8 \mathrm{~m}$}<smiles>CCOC(=O)C(C)(c1ccccc1)c1cc(O)c(O)c(OC)c1</smiles>

According to method A, 3-methoxycatechol $(140 \mathrm{mg}, 1.0 \mathrm{mmol}$ ) was reacted with ethyl phenylcyanoacetate $(189 \mathrm{mg}, 1.0 \mathrm{mmol})$ to give, after 30 min reaction time, compound $8 \mathrm{~m}$ (311 $\mathrm{mg}, 95 \%)$ as a brown oil after filtering through a small plug of silica, eluting with diethyl ether; ${ }^{1} \mathbf{H}$ NMR $\left(500 \mathrm{MHz}, \mathrm{CDCl}_{3}\right) \delta_{\mathrm{H}} 7.40-7.38(5 \mathrm{H}, \mathrm{m}, 5$ of $\mathrm{Ar}-\mathrm{H}), 6.62(1 \mathrm{H}, \mathrm{d}, \mathrm{J}=2.0 \mathrm{~Hz}$, $\operatorname{Ar}-\mathrm{H}), 6.55(1 \mathrm{H}, \mathrm{d}, \mathrm{J}=2.0 \mathrm{~Hz}, \mathrm{Ar}-\mathrm{H}), 5.52(1 \mathrm{H}, \mathrm{s} . \mathrm{OH}), 5.39(1 \mathrm{H}, \mathrm{s}, \mathrm{OH}), 4.34(2 \mathrm{H}, \mathrm{q}, \mathrm{J}=7.0$ $\left.\mathrm{Hz}, \mathrm{O}-\mathrm{CH}_{2}-\mathrm{CH}_{3}\right), 3.82\left(3 \mathrm{H}, \mathrm{s}, \mathrm{O}-\mathrm{CH}_{3}\right), 1.32\left(3 \mathrm{H}, \mathrm{t}, \mathrm{J}=7.0 \mathrm{~Hz}, \mathrm{O}-\mathrm{CH}_{2}-\mathrm{CH}_{3}\right) ;{ }^{13} \mathrm{C}^{\mathrm{NMR}}(125$ 
$\left.\mathrm{MHz}, \mathrm{CDCl}_{3}\right) \delta_{\mathrm{C}} 167.2,147.0,143.8,135.7,132.8,128.9,128.8,127.9,127.2,118.8,109.2$ 103.2, 63.7, 58.5, 56.2, 13.9; IR $v_{\max } / \mathrm{cm}^{-1} 3431,1745,1615,1516,1452,1305,1223,1096$; MS $(\mathrm{Cl}+) \mathrm{m} / \mathrm{z}$ (relative intensity \%) $345\left(\mathrm{M}+\mathrm{NH}_{4}{ }^{+}, 100 \%\right)$; HRMS (ES+): calcd. for $\mathrm{C}_{18} \mathrm{H}_{21} \mathrm{O}_{5} \mathrm{~N}_{2}\left(\mathrm{M}+\mathrm{NH}_{4}{ }^{+}\right) 345.1445$, found 345.1436.

\subsubsection{Synthesis and characterization of compound 8n}<smiles>COc1cc(C2(C#N)CCCCCC2=O)cc(O)c1O</smiles>

According to method A, 3-methoxycatechol $(70 \mathrm{mg}, 0.5 \mathrm{mmol}$ ) was reacted with 2oxocycloheptanecarbonitrile (69 $\mathrm{mg}, 0.5 \mathrm{mmol}$ ) to give, after $4 \mathrm{~h}$ reaction time, compound $8 \mathrm{n}$ (107 $\mathrm{mg}, 78 \%$ ) as an off-white solid after filtering through a small plug of silica, eluting with diethyl ether; ${ }^{1} \mathrm{H}$ NMR $(500 \mathrm{MHz}, \mathrm{DMSO}) \delta_{\mathrm{H}} 9.28(1 \mathrm{H}, \mathrm{s}, \mathrm{OH}), 8.66(1 \mathrm{H}, \mathrm{s}, \mathrm{OH}), 6.54(2 \mathrm{H}, \mathrm{s}, 2$ of $\mathrm{Ar}-\mathrm{H}), 3.78\left(3 \mathrm{H}, \mathrm{s}, \mathrm{OCH}_{3}\right), 2.87-2.81\left(1 \mathrm{H}, \mathrm{m}, 1 \mathrm{H}\right.$ of $\left.\mathrm{CH}_{2}\right), 2.55-2.43\left(2 \mathrm{H}, \mathrm{m}, 2\right.$ of $\left.\mathrm{CH}_{2}\right), 2.32-$ $2.28\left(1 \mathrm{H}, \mathrm{m}, 1\right.$ of $\left.\mathrm{CH}_{2}\right), 2.12-2.09\left(1 \mathrm{H}, \mathrm{m}, 1\right.$ of $\left.\mathrm{CH}_{2}\right), 1.99-1.95\left(1 \mathrm{H}, \mathrm{m}, 1\right.$ of $\left.\mathrm{CH}_{2}\right), 1.83(1 \mathrm{H}, \mathrm{d}$, $\mathrm{J}=14.0 \mathrm{~Hz}, 1$ of $\left.\mathrm{CH}_{2}\right), 1.61-1.52\left(1 \mathrm{H}, \mathrm{m}, 1\right.$ of $\left.\mathrm{CH}_{2}\right) 1.40-1.28\left(2 \mathrm{H}, \mathrm{m}, 2\right.$ of $\left.\mathrm{CH}_{2}\right) ;{ }^{13} \mathrm{C} \mathrm{NMR} \mathrm{(75}$ $\mathrm{MHz}, \mathrm{DMSO}) \delta_{\mathrm{C}} 204.6,148.7,146.1,134.8,124.2,120.9,107.5,101.7,59.5,56.1,56.1$, 38.9, 33.0, 28.7, 26.6, 25.0; IR $v_{\max } / \mathrm{cm}^{-1} 3333,2934,1720,1700,1612,1519,1302,1107$, 938; MPT $155-158^{\circ} \mathrm{C}$; MS $(\mathrm{Cl}+) \mathrm{m} / \mathrm{z}$ (relative intensity \%) $293\left(\mathrm{M}+\mathrm{NH}_{4}{ }^{+}, 100 \%\right)$; HRMS (ES+): calcd. for $\mathrm{C}_{15} \mathrm{H}_{21} \mathrm{O}_{4} \mathrm{~N}\left(\mathrm{M}+\mathrm{NH}_{4}{ }^{+}\right)$293.1496, found 293.1496.

\subsubsection{Synthesis and characterization of compound 80}

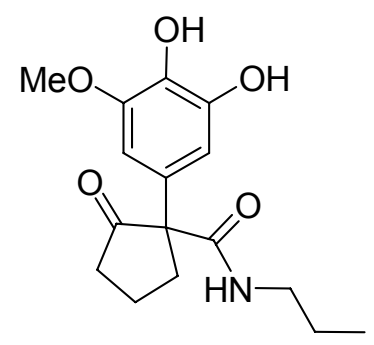

According to method A, 3-methoxycatechol $(28 \mathrm{mg}, 0.2 \mathrm{mmol})$ was reacted with 2-oxo-Npropylcyclopentanecarboxamide ( $34 \mathrm{mg}, 0.2 \mathrm{mmol}$ ) to give, after $6 \mathrm{~h}$ reaction time, compound 80 (48 mg, 78\%) as a colourless oil after chromatography on silica, eluting with petroleum ether containing 50-70\% diethyl ether; ${ }^{1} \mathrm{H}$ NMR $\left(500 \mathrm{MHz}\right.$, DMSO) $\delta_{\mathrm{H}} 6.61(1 \mathrm{H}, \mathrm{d}, \mathrm{J}=1.0 \mathrm{~Hz}$ Ar-H), $6.58(1 \mathrm{H}, \mathrm{s}, \mathrm{NH}), 6.48(1 \mathrm{H}, \mathrm{d}, \mathrm{J}=1.0 \mathrm{~Hz}, \mathrm{Ar}-\mathrm{H}), 6.03(1 \mathrm{H}, \mathrm{s}, \mathrm{OH}), 5.53(1 \mathrm{H}, \mathrm{s}, \mathrm{OH})$, $3.86\left(3 \mathrm{H}, \mathrm{s}, \mathrm{OCH}_{3}\right), 3.17\left(2 \mathrm{H}\right.$, apparent q, J = $\left.6.5 \mathrm{~Hz}, \mathrm{NCH}_{2} \mathrm{CH}_{2} \mathrm{CH}_{3}\right), 2.88(1 \mathrm{H}$, ddd, J = 14.0, 8.5, $6.5 \mathrm{~Hz}, 1 \mathrm{H}$ of $\left.\mathrm{CH}_{2}\right), 2.46-2.40\left(3 \mathrm{H}, \mathrm{m}, 3\right.$ of $\left.\mathrm{CH}_{2}\right), 1.95(1 \mathrm{H}$ apparent qd, $\mathrm{J}=13.0,6.5 \mathrm{~Hz}$, 
$1 \mathrm{H}$ of $\left.\mathrm{CH}_{2}\right), 1.84-1.75\left(1 \mathrm{H}, \mathrm{m}, 1 \mathrm{H}\right.$ of $\left.\mathrm{CH}_{2}\right), 1.46(2 \mathrm{H}$, apparent sextet, $\mathrm{J}=7.0 \mathrm{~Hz}$, $\left.\mathrm{NCH}_{2} \mathrm{CH}_{2} \mathrm{CH}_{3}\right), 0.83\left(3 \mathrm{H}, \mathrm{t}, \mathrm{J}=7.0 \mathrm{~Hz}, \mathrm{NCH}_{2} \mathrm{CH}_{2} \mathrm{CH}_{3}\right) ;{ }^{13} \mathrm{C} \mathrm{NMR}\left(75 \mathrm{MHz}, \mathrm{CDCl}_{3}\right) \delta_{\mathrm{C}} 217.9$, 169.6, 147.3, 144.2, 132.3, 130.0, 107.4, 101.7, 64.5, 56.2, 41.5, 39.2, 35.8, 22.5, 18.8, 11.2; IR $v_{\max } / \mathrm{cm}^{-1} 3369,2963,2935,2877,1726,1652,1520$; MS (ES-) $\mathrm{m} / \mathrm{z}$ (relative intensity \%) $306\left(\mathrm{M}-\mathrm{H}^{+}, 100 \%\right)$; HRMS (EI+): calcd. for $\mathrm{C}_{16} \mathrm{H}_{21} \mathrm{O}_{5} \mathrm{~N}\left(\mathrm{M}^{+}\right)$307.1414, found 307.1429 .

\subsubsection{Synthesis and characterization of compound 8p}<smiles>COc1cc(C2(C(=O)OC(C(F)(F)F)C(F)(F)F)CCCC2=O)cc(O)c1O</smiles>

According to method A, 3-methoxycatechol (14 mg, $0.1 \mathrm{mmol}$ ) was reacted with 1,1,1,3,3,3hexafluoropropan-2-yl 2-oxocyclopentanecarboxylate $(28 \mathrm{mg}, 0.1 \mathrm{mmol})$ to give, after $2 \mathrm{~h}$ reaction time, compound $\mathbf{8 p}(37 \mathrm{mg}, 89 \%)$ as an off-white solid after chromatography on silica, eluting with petroleum ether containing $50 \%$ diethyl ether; ${ }^{1} \mathrm{H} \mathrm{NMR}\left(500 \mathrm{MHz}, \mathrm{CDCl}_{3}\right) \delta_{\mathrm{H}}$ $6.63(1 \mathrm{H}, \mathrm{d}, \mathrm{J}=1.5 \mathrm{~Hz}, \operatorname{Ar}-\mathrm{H}), 6.57(1 \mathrm{H}, \mathrm{d}, \mathrm{J}=1.5 \mathrm{~Hz}, \operatorname{Ar}-\mathrm{H}), 5.72(1 \mathrm{H}, \mathrm{h} ., \mathrm{J}=6.0 \mathrm{~Hz}$, $\left.\mathrm{CH}\left(\mathrm{CF}_{3}\right)_{2}\right), 3.86\left(3 \mathrm{H}, \mathrm{s}, \mathrm{OCH}_{3}\right), 2.79-2.73\left(1 \mathrm{H}, \mathrm{m}, 1\right.$ of $\left.\mathrm{CH}_{2}\right), 2.70-2.65\left(1 \mathrm{H}, \mathrm{m}, 1\right.$ of $\left.\mathrm{CH}_{2}\right)$, 2.52-2.38 $\left(2 \mathrm{H}, \mathrm{m}, \mathrm{CH}_{2}\right), 2.08-1.92\left(2 \mathrm{H}, \mathrm{m}, \mathrm{CH}_{2}\right) ;{ }^{13} \mathrm{C}$ NMR $\left(1255 \mathrm{MHz}, \mathrm{CDCl}_{3}\right) \delta_{\mathrm{C}} 209.5$, 168.1, 147.0, 143.9, 132.5, 125.1, $121.3(\mathrm{~m}), 119.1(\mathrm{~m}), 107.8,103.1,67.1$ (h. J = $35 \mathrm{~Hz}$ ), 63.6, 56.2, 37.2, 34.1, 19.2; IR $v_{\max } / \mathrm{cm}^{-1} 3460,2973,2854,2370,1775,1747,1618$; MPT 104-105 ${ }^{\circ} \mathrm{C}$; MS (EI) m/z (relative intensity \%) 415 (M - $\left.\mathrm{H}^{+}, 100 \%\right)$; HRMS (El+): calcd. for $\mathrm{C}_{16} \mathrm{H}_{14} \mathrm{O}_{6} \mathrm{~F}_{6}\left(\mathrm{M}^{+}\right)$416.0689, found 416.0709.

\subsection{Synthesis of enantioenriched adduct 11}

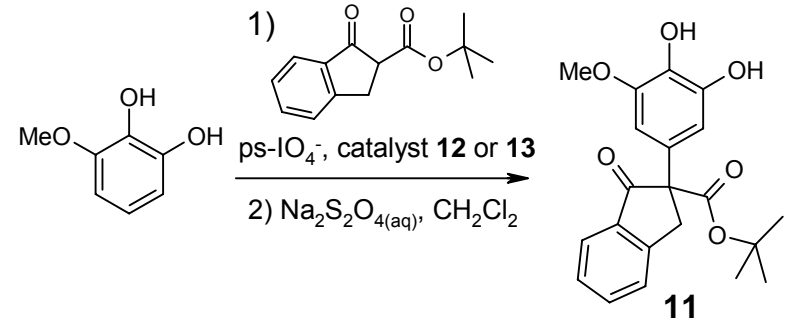

11

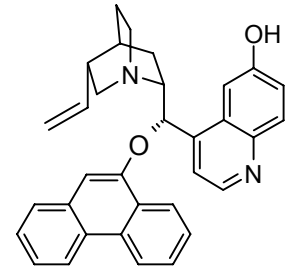

catalyst 12

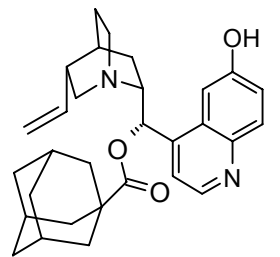

catalyst 13

According to method $A$, where ps-BEMP is replaced by catalyst 12 (4.8 mg, $10 \mathrm{~mol} \%), 3-$ methoxycatechol (14 mg, $0.1 \mathrm{mmol}$ ) was reacted with 2-(2,2-dimethylpropanoyl)indan-1-one (23 $\mathrm{mg}, 0.1 \mathrm{mmol}$ ) to give, after $2 \mathrm{~h}$ reaction time, compound 11 (30 mg, 81\%) as an off-white 
solid after chromatography on silica eluting with petroleum ether containing $50-70 \%$ diethyl ether, in 74\% ee, determined by HPLC analysis [Chiralpak AD, hexane/iso-propanol, 4:1, 1.0 $\mathrm{mLmin}^{-1}, \lambda=230 \mathrm{~nm}, \mathrm{t}$ (minor) $=20.16 \mathrm{~min}, \mathrm{t}$ (major) $=26.56 \mathrm{~min}$ ] by comparison to $\mathrm{a}$ racemic sample prepared according to method $\mathrm{A} ;[\alpha]_{\mathrm{D}}{ }^{24}=-1.63$ (c $\left.0.81, \mathrm{CHCl}_{3}\right) ;{ }^{1} \mathbf{H}$ NMR $\left(500 \mathrm{MHz}, \mathrm{CDCl}_{3}\right) \delta_{\mathrm{H}} 7.80(1 \mathrm{H}, \mathrm{d}, \mathrm{J}=7.5 \mathrm{~Hz}, \mathrm{Ar}-\mathrm{H}), 7.61(1 \mathrm{H}, \mathrm{t}, \mathrm{J}=7.5 \mathrm{~Hz}, \operatorname{Ar}-\mathrm{H}), 7.48(1 \mathrm{H}, \mathrm{d}$, $\mathrm{J}=7.5 \mathrm{~Hz}, \operatorname{Ar}-\mathrm{H}), 7.39(1 \mathrm{H}, \mathrm{t}, \mathrm{J}=7.5, \operatorname{Ar}-\mathrm{H}), 6.71(1 \mathrm{H}, \mathrm{d}, \mathrm{J}=2.0 \mathrm{~Hz}, \operatorname{Ar}-\mathrm{H}), 6.61(1 \mathrm{H}, \mathrm{d}, \mathrm{J}=$ 2.0, Hz, Ar-H), $5.45(1 \mathrm{H}, \mathrm{s}, \mathrm{OH}), 5.41(1 \mathrm{H}, \mathrm{s}, \mathrm{OH}), 4.05\left(1 \mathrm{H}, \mathrm{d}, \mathrm{J}=17.0 \mathrm{~Hz}, 1 \mathrm{H}\right.$ of $\left.\mathrm{CH}_{2}\right), 3.85$ $\left(3 \mathrm{H}, \mathrm{s}, \mathrm{OCH}_{3}\right), 3.56\left(1 \mathrm{H}, \mathrm{d}, \mathrm{J}=17.0 \mathrm{~Hz}, 1 \mathrm{H}\right.$ of $\left.\mathrm{CH}_{2}\right), 1.39\left(9 \mathrm{H}, \mathrm{s}, \mathrm{C}\left(\mathrm{CH}_{3}\right)_{3}\right) ;{ }^{13} \mathrm{C} \mathrm{NMR}(75 \mathrm{MHz}$, $\left.\mathrm{CDCl}_{3}\right) \delta_{\mathrm{C}} 200.6,169.6,152.0,146.7,143.6,135.3,135.1,131.9,130.1,127.7,126.0,124.9$, 108.0, 103.1, 82.4, 65.4, 56.1, 40.4, 27.7; IR $v_{\max } / \mathrm{cm}^{-1} 3416,2966,1729,1713,1607,1517$, 1463, 1370, 1254, 1153, 1096, 842; MPT 144-147 ${ }^{\circ} \mathrm{C}$; MS (Cl+) m/z (relative intensity \%) 332 $\left(\mathrm{M}-{ }^{\mathrm{t}} \mathrm{Bu}+\mathrm{NH}_{4}{ }^{+}, 100 \%\right), 388\left(\mathrm{M}+\mathrm{NH}_{4}{ }^{+}, 15 \%\right)$; HRMS (ES+): calcd. for $\mathrm{C}_{21} \mathrm{H}_{26} \mathrm{O}_{6} \mathrm{~N}\left(\mathrm{M}+\mathrm{NH}_{4}{ }^{+}\right)$ 388.1755, found 388.1763 .

Similarly, replacing ps-BEMP with catalyst $13(4.7 \mathrm{mg}, 10 \mathrm{~mol} \%)$ gave, after $2 \mathrm{~h}$ reaction time, compound 11 (31 mg, 84\%) in 81\% ee, determined by HPLC analysis [Chiralpak AD, hexane/iso-propanol, 4:1,1.0 $\mathrm{mLmin}^{-1}, \lambda=230 \mathrm{~nm}, \mathrm{t}$ (minor) $=19.82 \mathrm{~min}, \mathrm{t}$ (major) $=25.83$ $\min ] ; ;[\alpha]_{D}^{24}=-1.61\left(\mathrm{c} 0.87, \mathrm{CHCl}_{3}\right)$.

\subsection{Preparation of catalysts}

Catalysts were prepared according to the method of Deng and co-workers ${ }^{3}$.

Catalyst 13: $[\alpha]_{D}{ }^{24}=+27.03\left(\mathrm{c} 1.45, \mathrm{CHCl}_{3}\right) ;{ }^{1} \mathrm{H}$ NMR $(500 \mathrm{MHz}, \mathrm{MeOD}) \delta_{\mathrm{H}} 8.60(1 \mathrm{H}, \mathrm{d}, \mathrm{J}=$ $4.6 \mathrm{~Hz}, \operatorname{Ar}-\mathrm{H}), 7.93(1 \mathrm{H}, \mathrm{d}, \mathrm{J}=9.1 \mathrm{~Hz}, \operatorname{Ar}-\mathrm{H}) 7.41-7.40(2 \mathrm{H}, \mathrm{m}, 2$ of $\operatorname{Ar}-\mathrm{H}), 7.37(1 \mathrm{H}, \mathrm{d}, \mathrm{J}=$ $9.1 \mathrm{~Hz}, \mathrm{Ar}-\mathrm{H}), 6.46(1 \mathrm{H}, \mathrm{d}, \mathrm{J}=4.2 \mathrm{~Hz}, \mathrm{CH}-\mathrm{O}-\mathrm{CO}), 6.12(1 \mathrm{H}, \mathrm{ddd}, \mathrm{J}=17.5,10.4,7.4 \mathrm{~Hz}$, $\left.\mathrm{CH}=\mathrm{CH}_{2}\right), 5.17-5.14\left(2 \mathrm{H}, \mathrm{m}, \mathrm{CH}_{2}=\mathrm{CH}\right), 3.33(1 \mathrm{H}$, br. s, 1 of $\mathrm{N}-\mathrm{CH}), 3.14-3.09(1 \mathrm{H}, \mathrm{m}, 1$ of N$\mathrm{CH}), 3.02-2.97(1 \mathrm{H}, \mathrm{m}, 1$ of $\mathrm{N}-\mathrm{CH}), 2.89-2.86(1 \mathrm{H}, \mathrm{m}, 1$ of $\mathrm{N}-\mathrm{CH}), 2.81-2.74(1 \mathrm{H}, \mathrm{m}, 1$ of $\mathrm{N}-\mathrm{CH}), 2.39(1 \mathrm{H}, \mathrm{dd}, \mathrm{J}=16.4,8 \mathrm{~Hz}$, aliphatic $\mathrm{CH}), 2.03(4 \mathrm{H}$, br. s, 4 aliphatic $\mathrm{CH}), 1.97(6 \mathrm{H}$, br. s, 6 aliphatic $\mathrm{CH}), 1.84(1 \mathrm{H}$, br. s, aliphatic $\mathrm{CH}), 1.77(6 \mathrm{H}, \mathrm{q}, \mathrm{J}=12.2 \mathrm{~Hz}, 6$ aliphatic $\mathrm{CH})$, $1.61(2 \mathrm{H}, \mathrm{t}, \mathrm{J}=6.3 \mathrm{~Hz}, 2$ aliphatic $\mathrm{CH}), 1.49(1 \mathrm{H}, \mathrm{s}$, aliphatic $\mathrm{CH}) ;{ }^{13} \mathrm{C} \mathrm{NMR}\left(75 \mathrm{MHz}, \mathrm{CDCl}_{3}\right)$ $\delta_{\mathrm{C}} 176.0,156.0,146.3,143.7,143.4,139.3,131.3,127.0,122.4,118.0,115.4,105.2,73.1$, 58.7, 49.5, 49.0, 40.8, 39.3, 38.8, 36.4, 27.8, 27.4, 25.6, 22.6; IR $v_{\max } / \mathrm{cm}^{-1} 2907,2850,1730$, 1618, 1468, 1454, 1226, 1182; MPT $182-185^{\circ} \mathrm{C}$ MS (ES+) m/z (relative intensity \%); 473 (M $\left.+\mathrm{H}^{+}, 100 \%\right), 495\left(\mathrm{M}+\mathrm{Na}^{+}, 70 \%\right), 427\left(\mathrm{M}+\mathrm{Na}^{+}+\mathrm{MeOH}, 70 \%\right)$; HRMS (ES+): calcd. for $\mathrm{C}_{30} \mathrm{H}_{37} \mathrm{O}_{3} \mathrm{~N}_{2}\left(\mathrm{M}+\mathrm{H}^{+}\right)$473.2789, found 473.2799. 


\section{References}

1. C. R. Harrison, P. Hodge, J. Chem. Soc. Perkin. Trans. I 1982, 509-511.

2. T. V. Hansen, L. Skattebøl, Tetrahedron Lett. 2005, 46, 3357-3358.

3. Y. Wang, X. Liu, L. Deng, J. Am. Chem. Soc. 2006, 128, 3928-3930 and references cited therein. 


\section{Spectra}

4.1 Spectra for compound $8 \mathbf{a}$

4.1.1 Proton NMR $\left(\mathrm{CDCl}_{3}\right)$

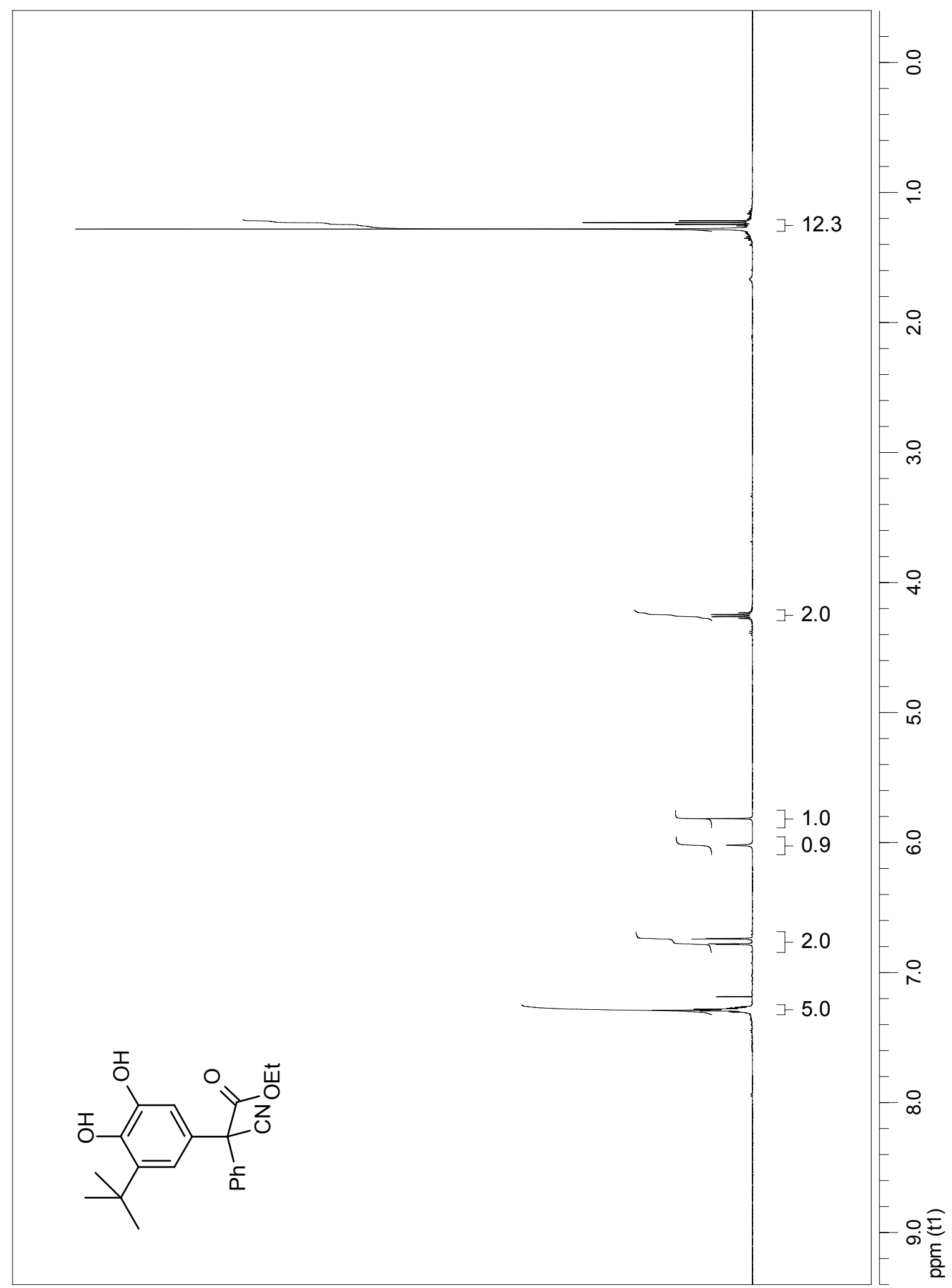




\subsubsection{Carbon-13 NMR $\left(\mathrm{CDCl}_{3}\right)$}

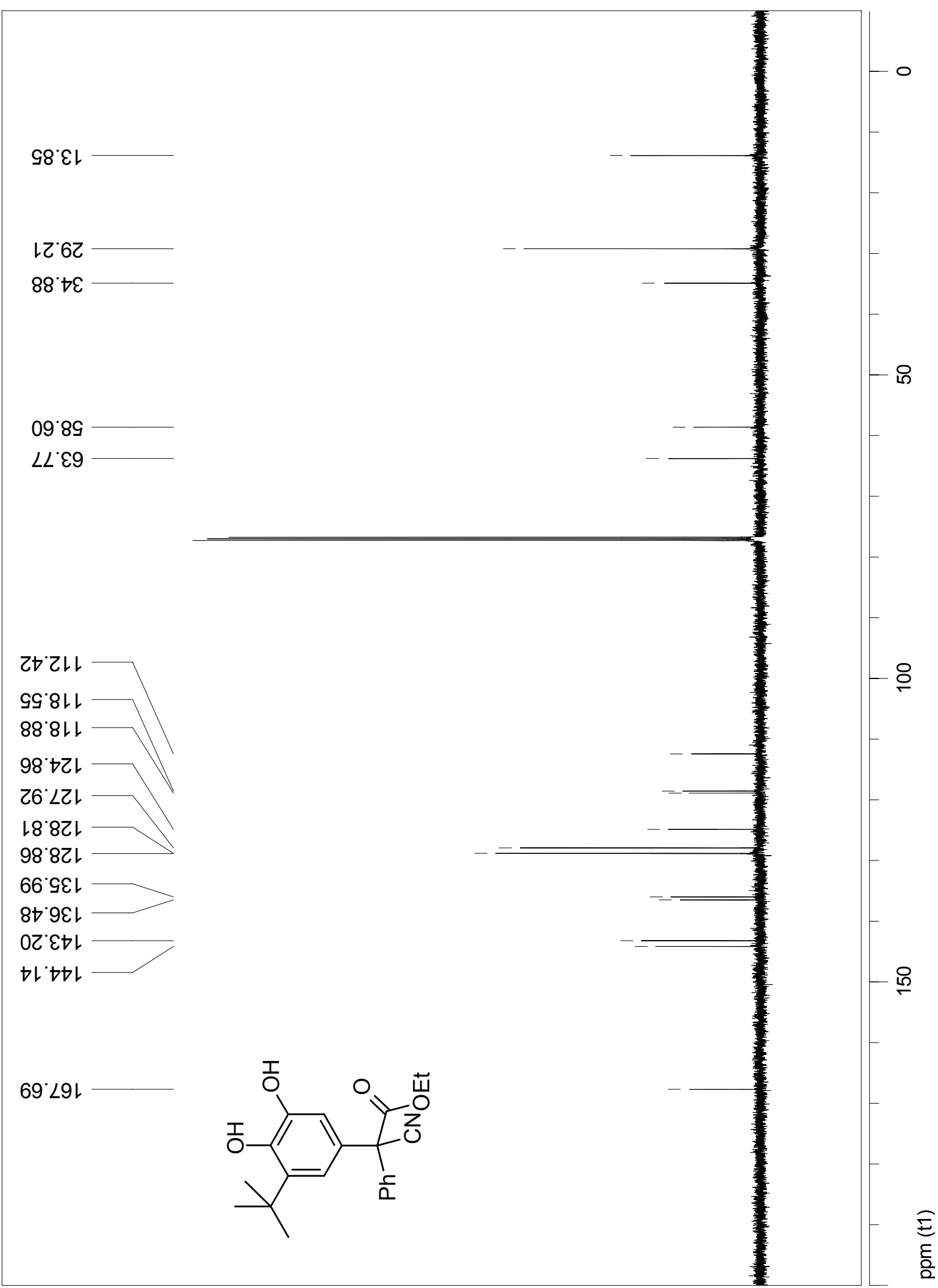


4.2 Spectra for compound $8 b$

4.2.1 Proton NMR $\left(\mathrm{CDCl}_{3}\right)$

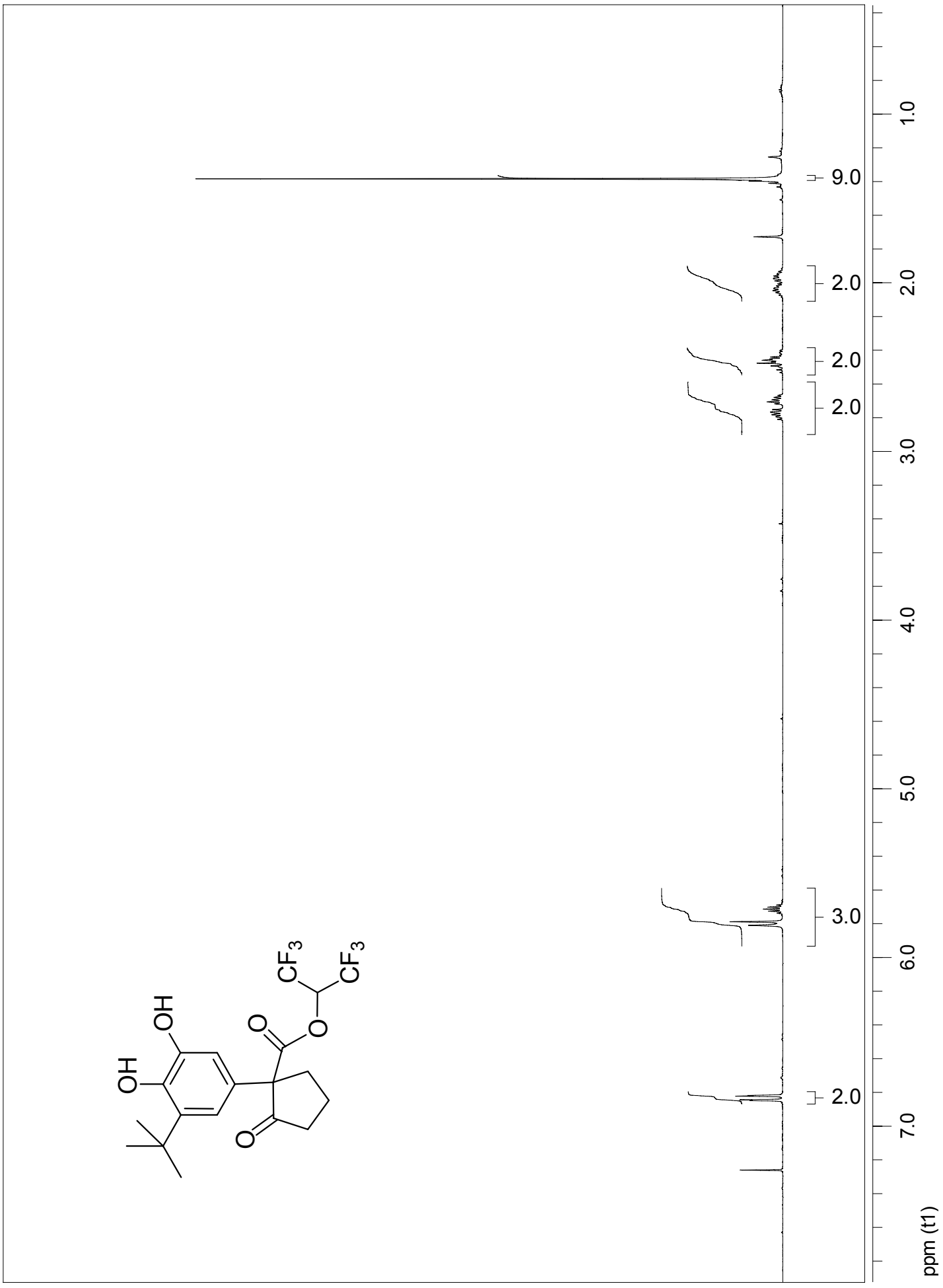


4.2.2 Carbon-13 NMR (MeOD)

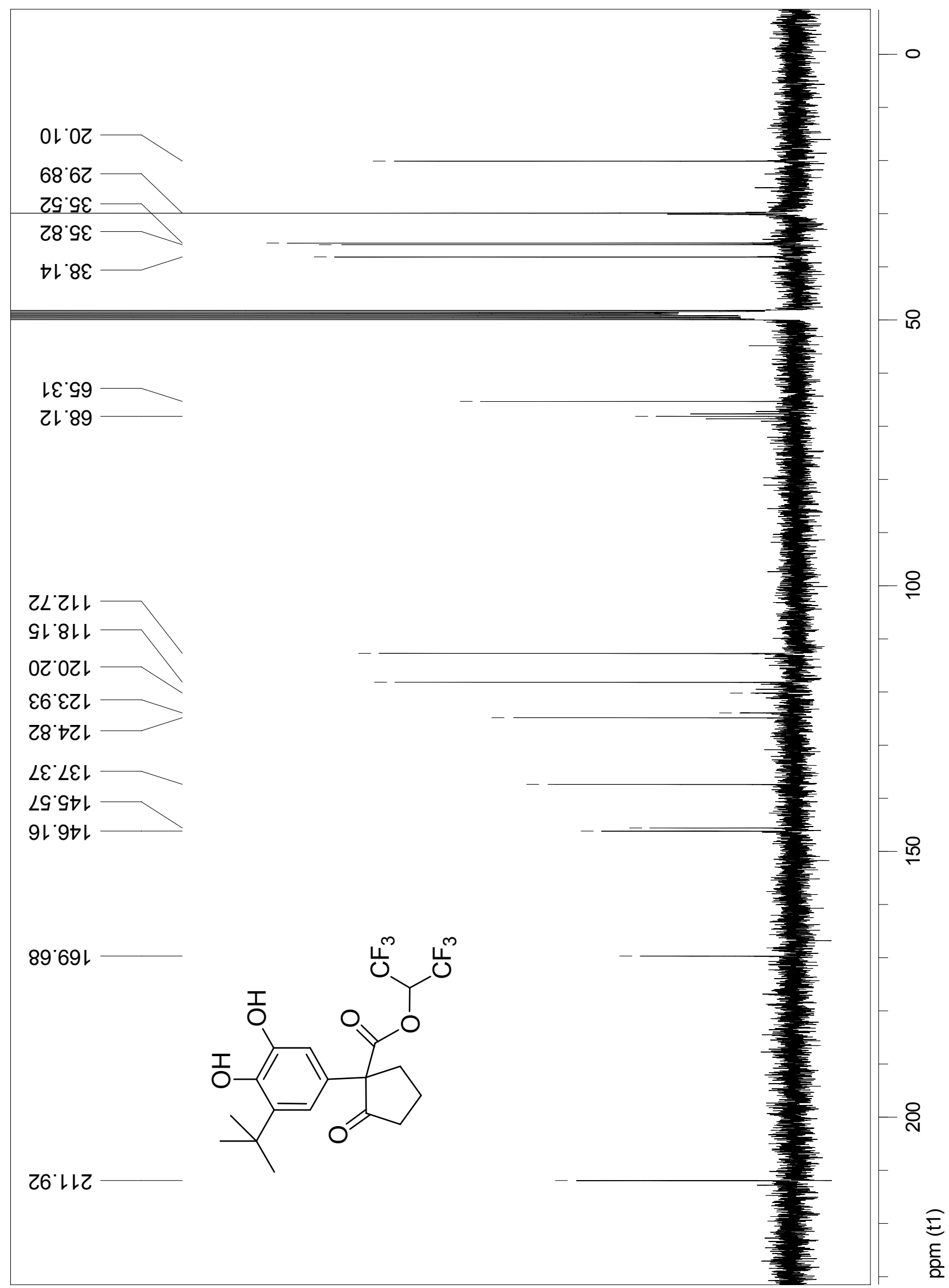


4.3 Spectra for compound 8c

4.3.1 Proton NMR $\left(\mathrm{CDCl}_{3}\right)$

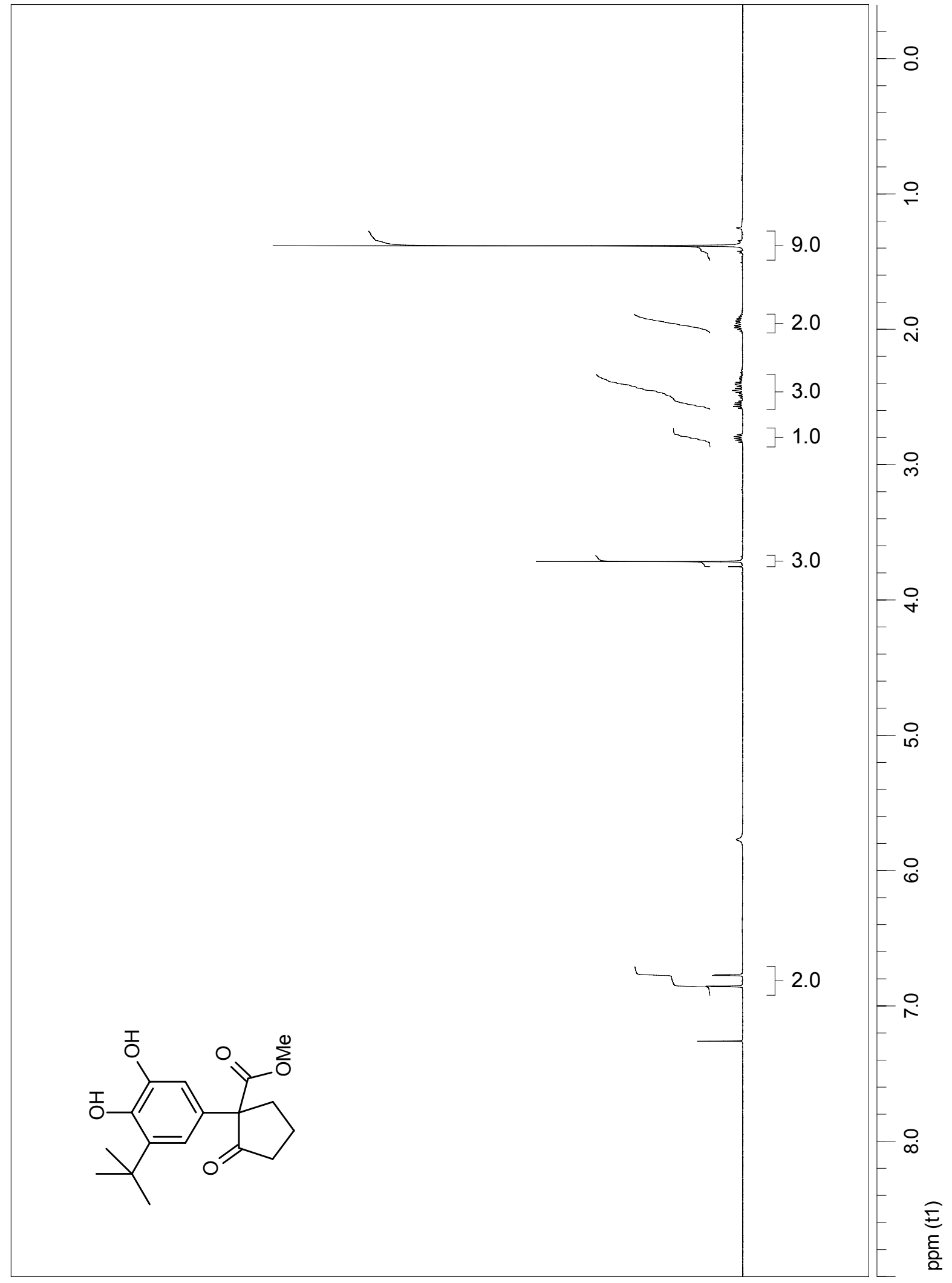


4.3.2 Carbon-13 NMR $\left(\mathrm{CDCl}_{3}\right)$

$\angle Z^{\prime} 6 \mathrm{~L}$

$9 \varepsilon^{\prime} 6 乙$

$\angle L ' \nabla \varepsilon$

$\varepsilon 8^{\circ} \nabla \varepsilon$

乙৪ $\angle \mathcal{E}$

レ๋๕G

$29^{\circ} \triangleright 9$

89`てレ

SI:LIL

6Z'SZL

$\angle 6 \cdot 9 \varepsilon L$

$00^{\circ} \varepsilon$ เト

$8 Z^{\circ} \varepsilon t レ$
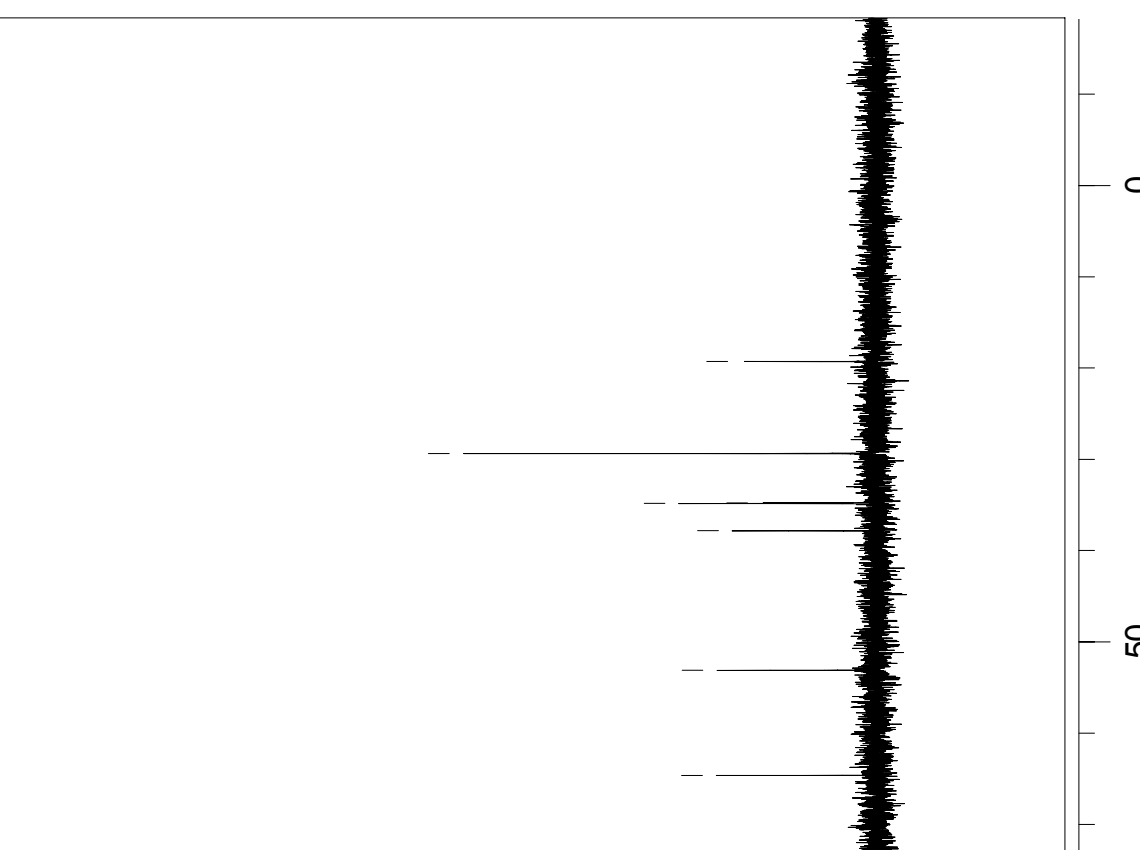

$-0$

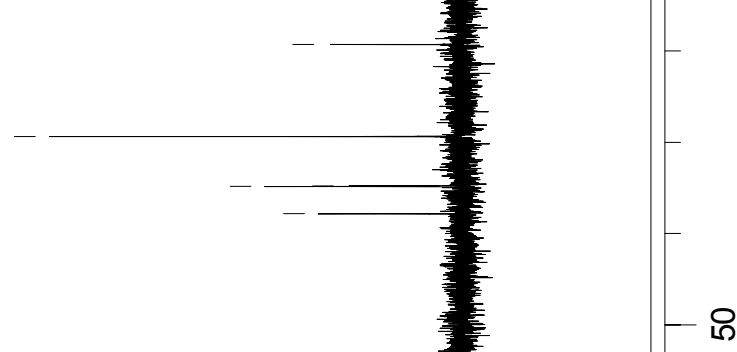

$88^{\circ} \cdot L L$<smiles>CC(C)(C)c1cc(C2(C([O])=O)CCCC2=O)cc([O])c1[O]</smiles>

$96 \cdot \varepsilon\llcorner 乙$ 
4.4 Spectra for compound 8d

4.4.1 Proton NMR $\left(\mathrm{CDCl}_{3}\right)$

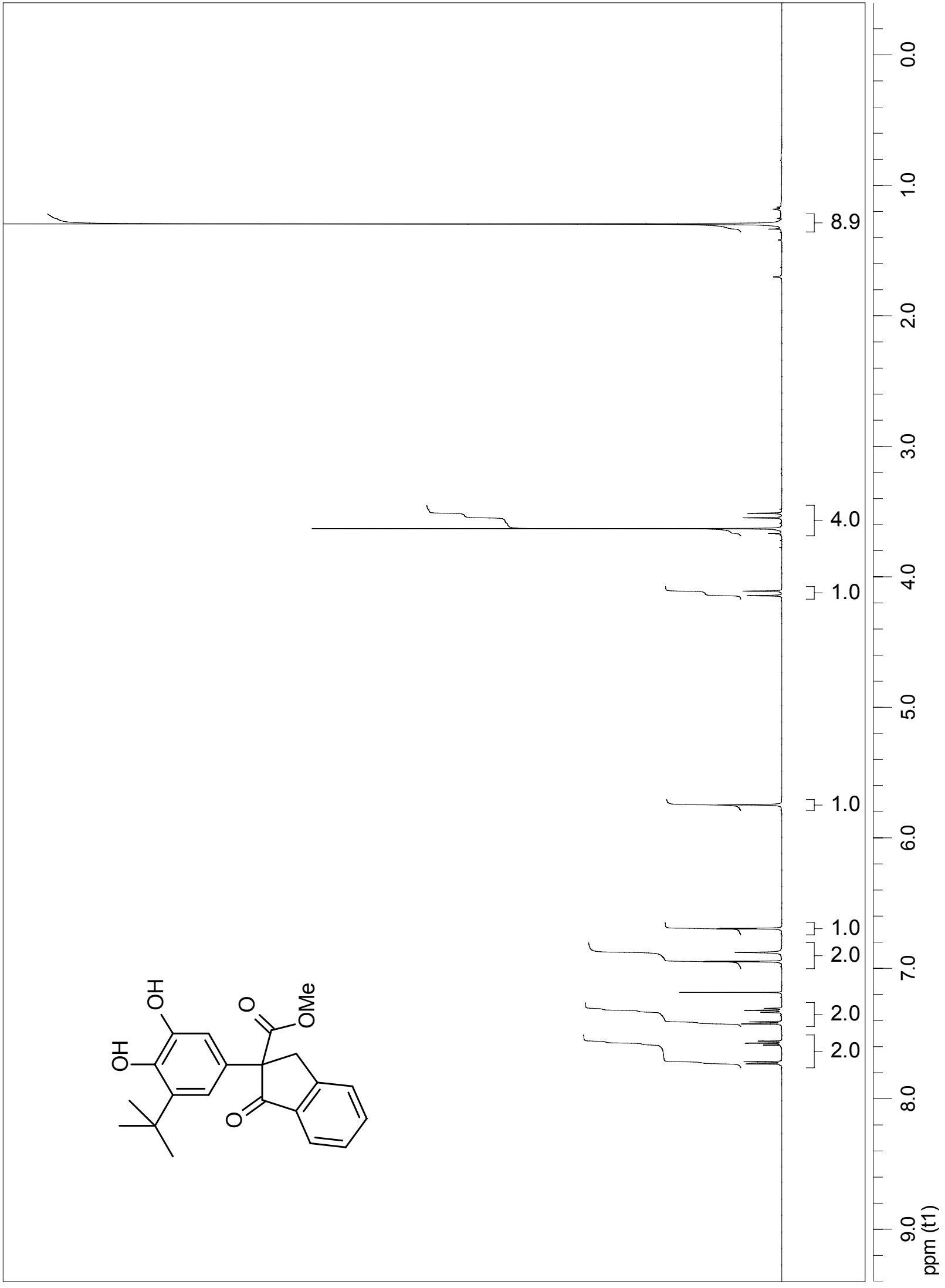




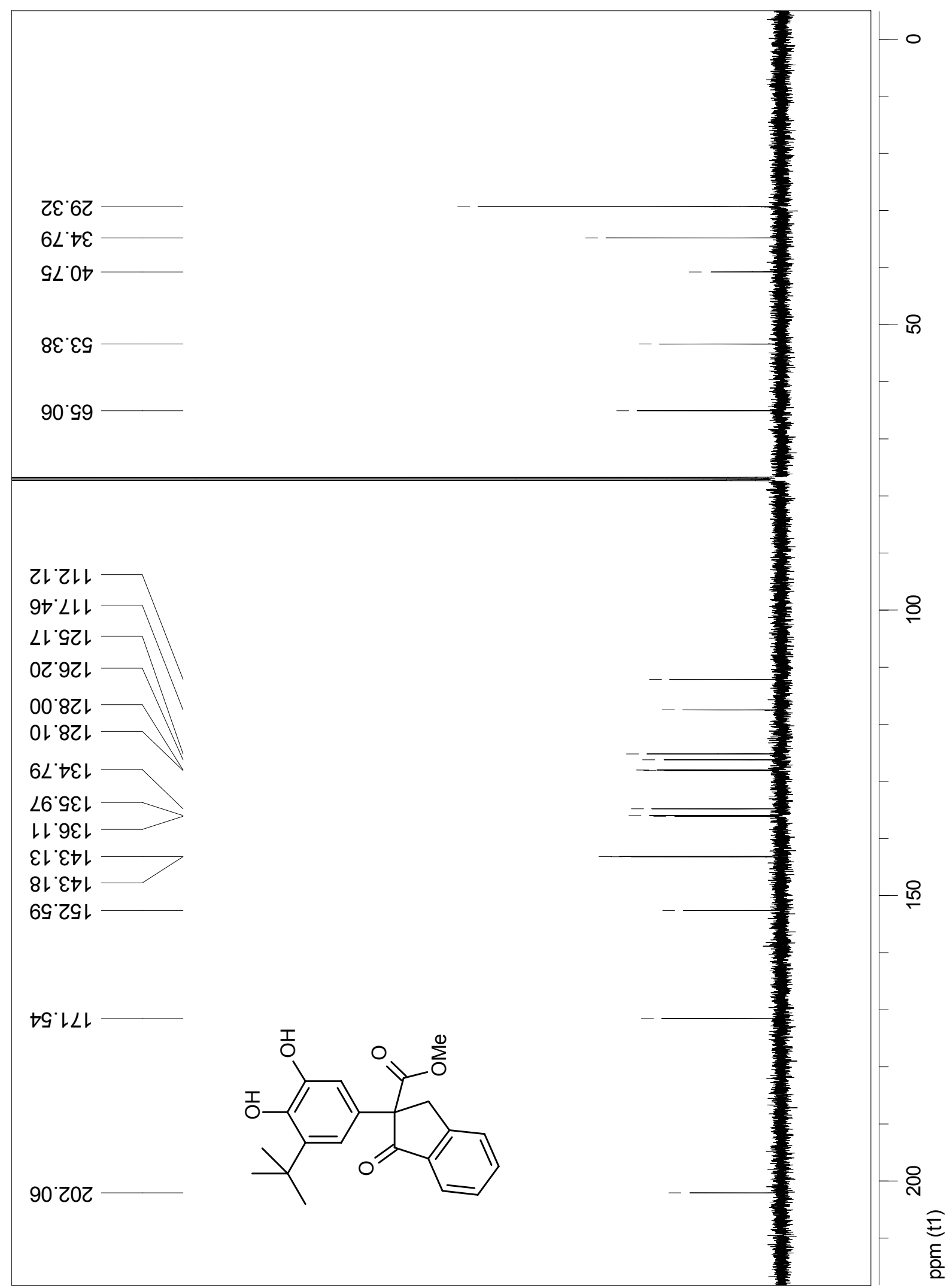


4.5 Spectra for compound 8e

4.5.1 Proton NMR $\left(\mathrm{CDCl}_{3}\right)$

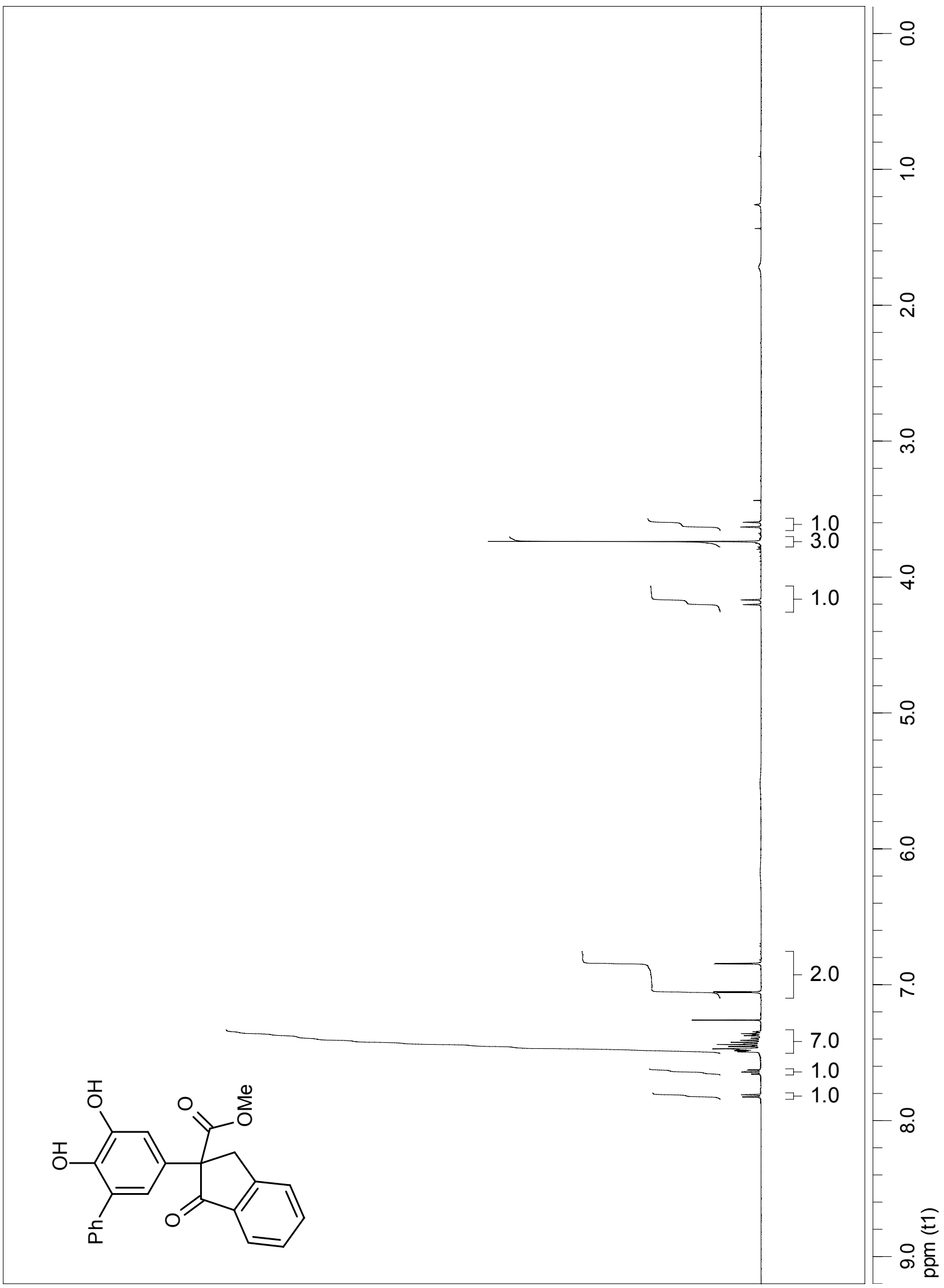




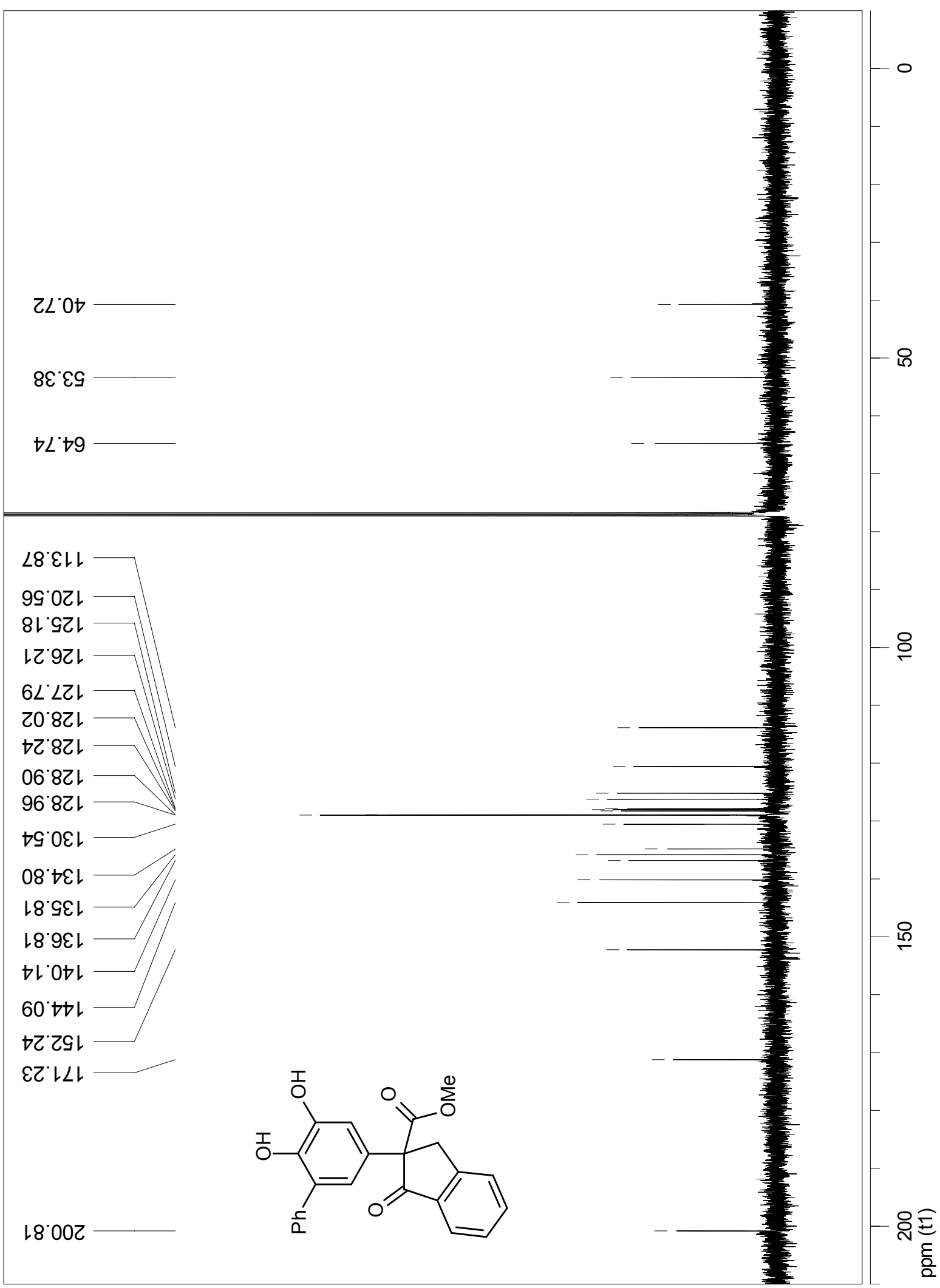


4.6 Spectra for compound $8 f$

4.6.1 Proton NMR $\left(\mathrm{CDCl}_{3}\right)$

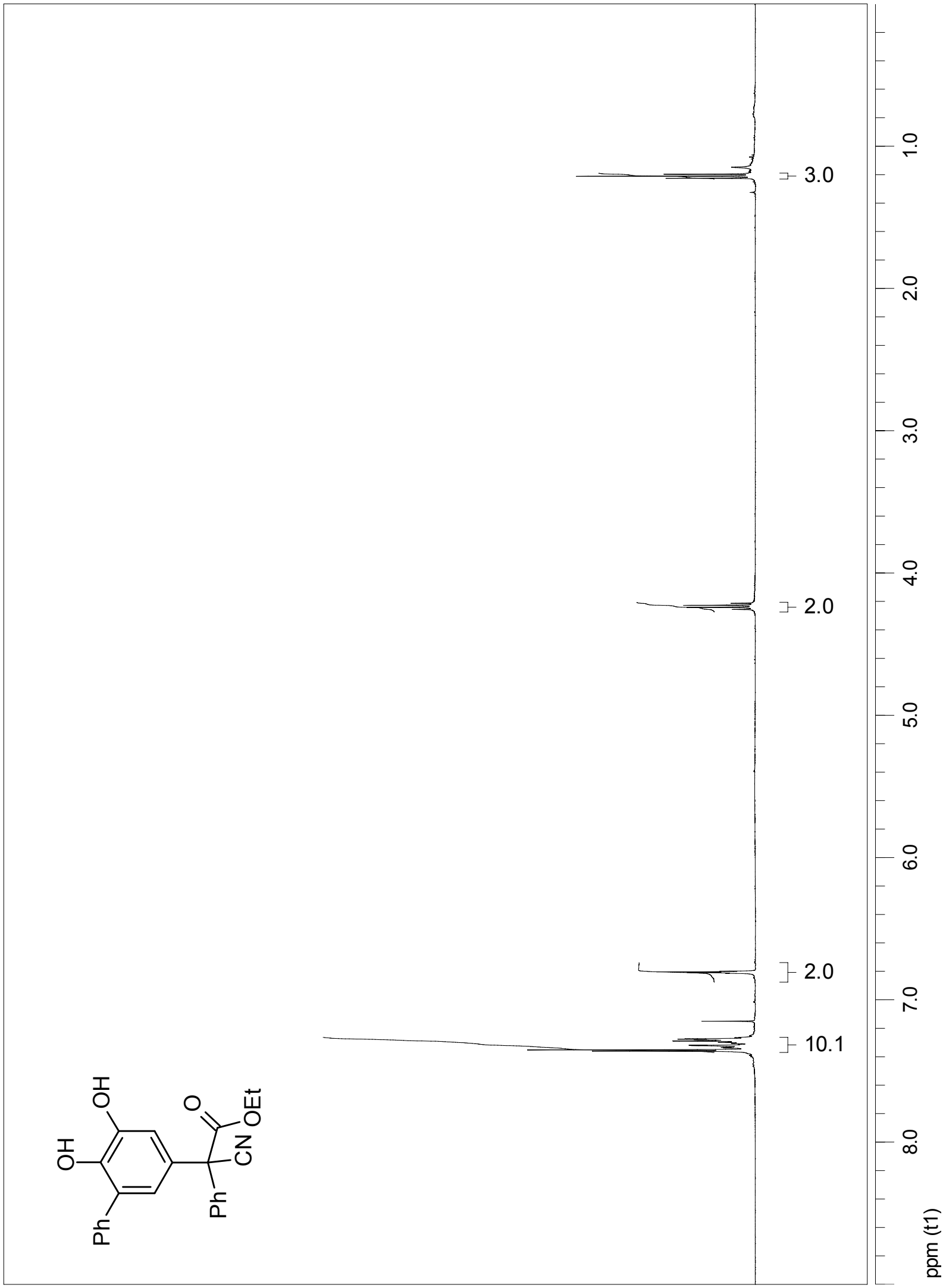




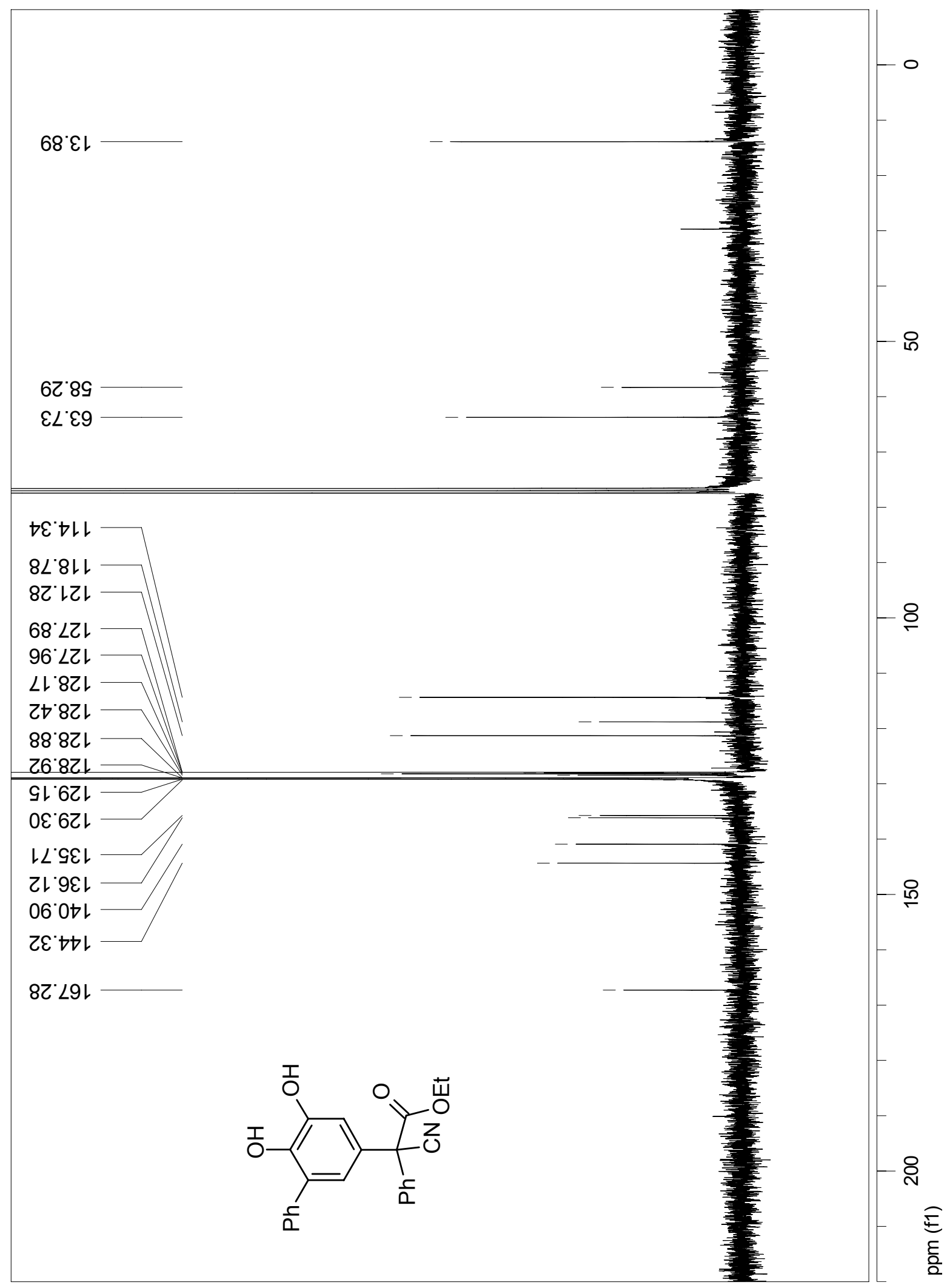


4.7 Spectra for compound $8 \mathrm{~g}$

4.7.1 Proton NMR $\left(\mathrm{CDCl}_{3}\right)$

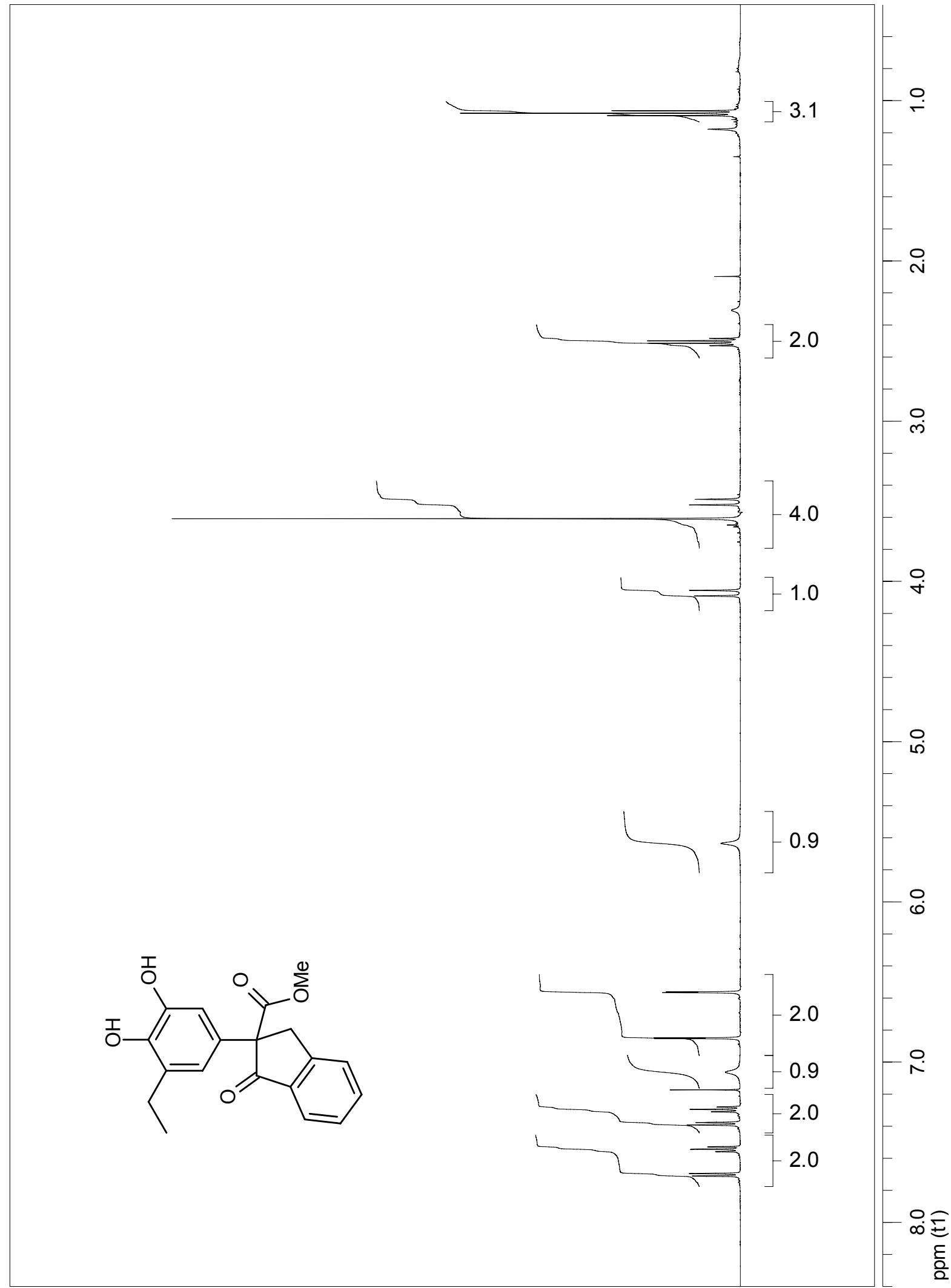


$66^{\circ} \varepsilon$

งเ`¿

LLOT

$9 \varepsilon^{\cdot} \varepsilon \varsigma$

$6 L ' t 9$

ยเ`てレ

†96 6 L

GL'GZL

6เ.9ZL

$66 \cdot \angle Z L$

$\varepsilon Z 6 Z$ L

LLOEL

$\nabla L \cdot \nabla \varepsilon L$

乙6. $\varsigma \varepsilon\llcorner$

96・เレ

レレEカレ

L†'ZGL

$\nabla G^{*} L L L$
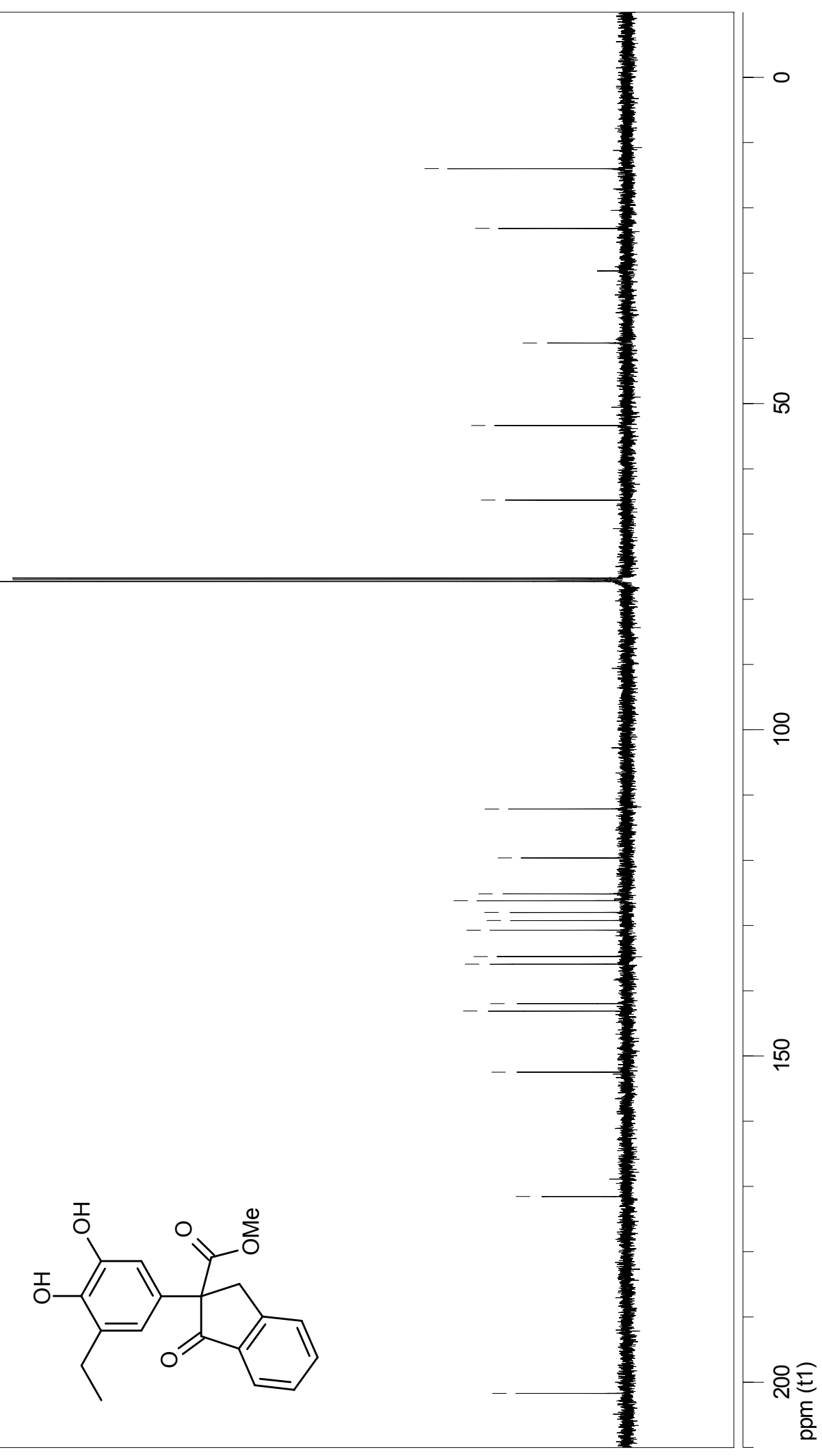

$0 L \cdot 10 Z$ 
4.8 Spectra for compound $8 \mathrm{~h}$

4.8.1 Proton NMR $\left(\mathrm{CDCl}_{3}\right)$

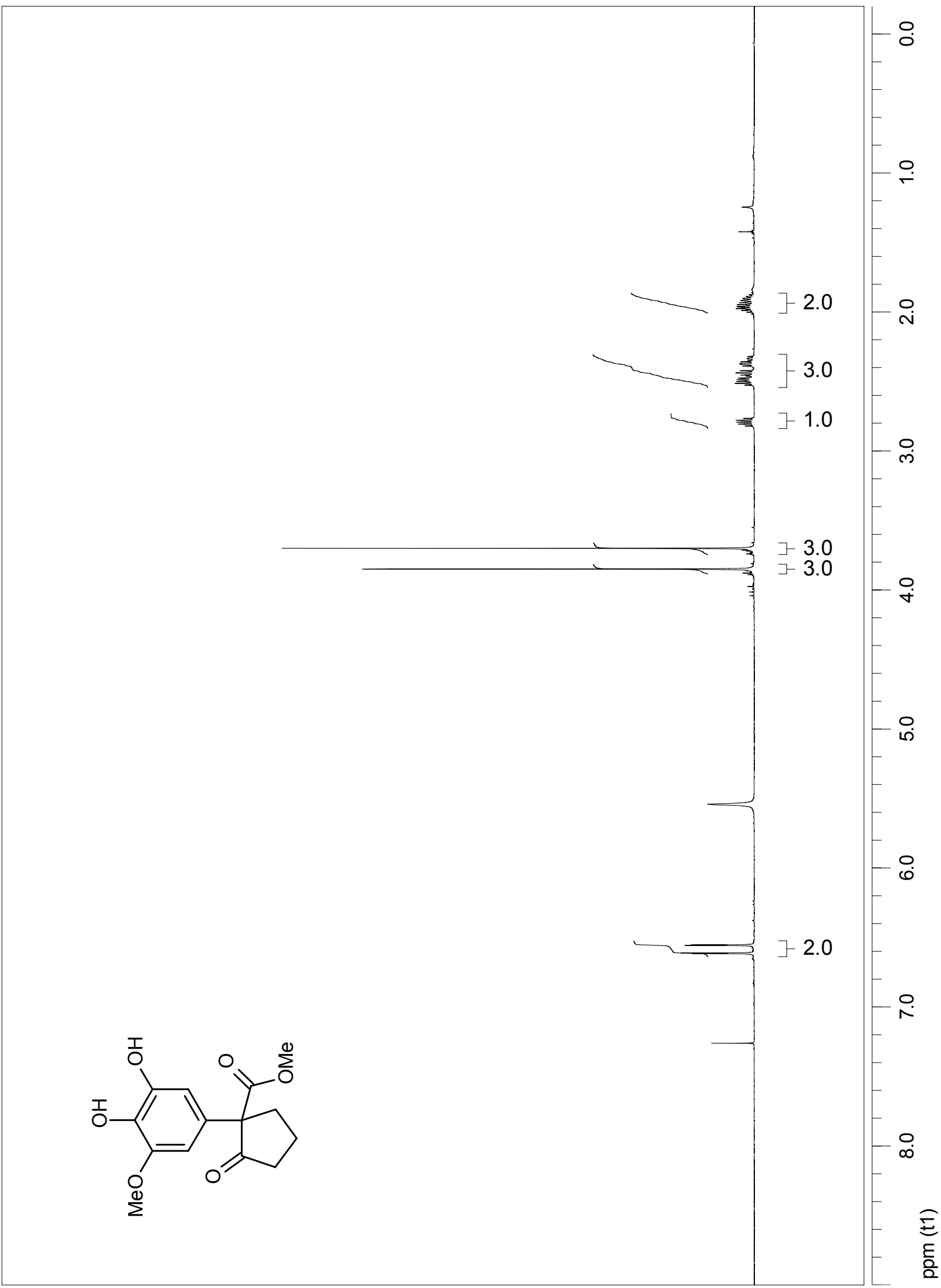


4.8.2 Carbon-13 NMR $\left(\mathrm{CDCl}_{3}\right)$

LZ6L

$88^{\circ} t \varepsilon$

$69^{\circ} \angle \varepsilon$

LO' $\varepsilon$

$9 l^{\circ} 99$

$0 \varepsilon^{*} \triangleright 9$

$68^{\circ} \mathrm{ZOL}$

$68^{\circ} \mathrm{LOL}$

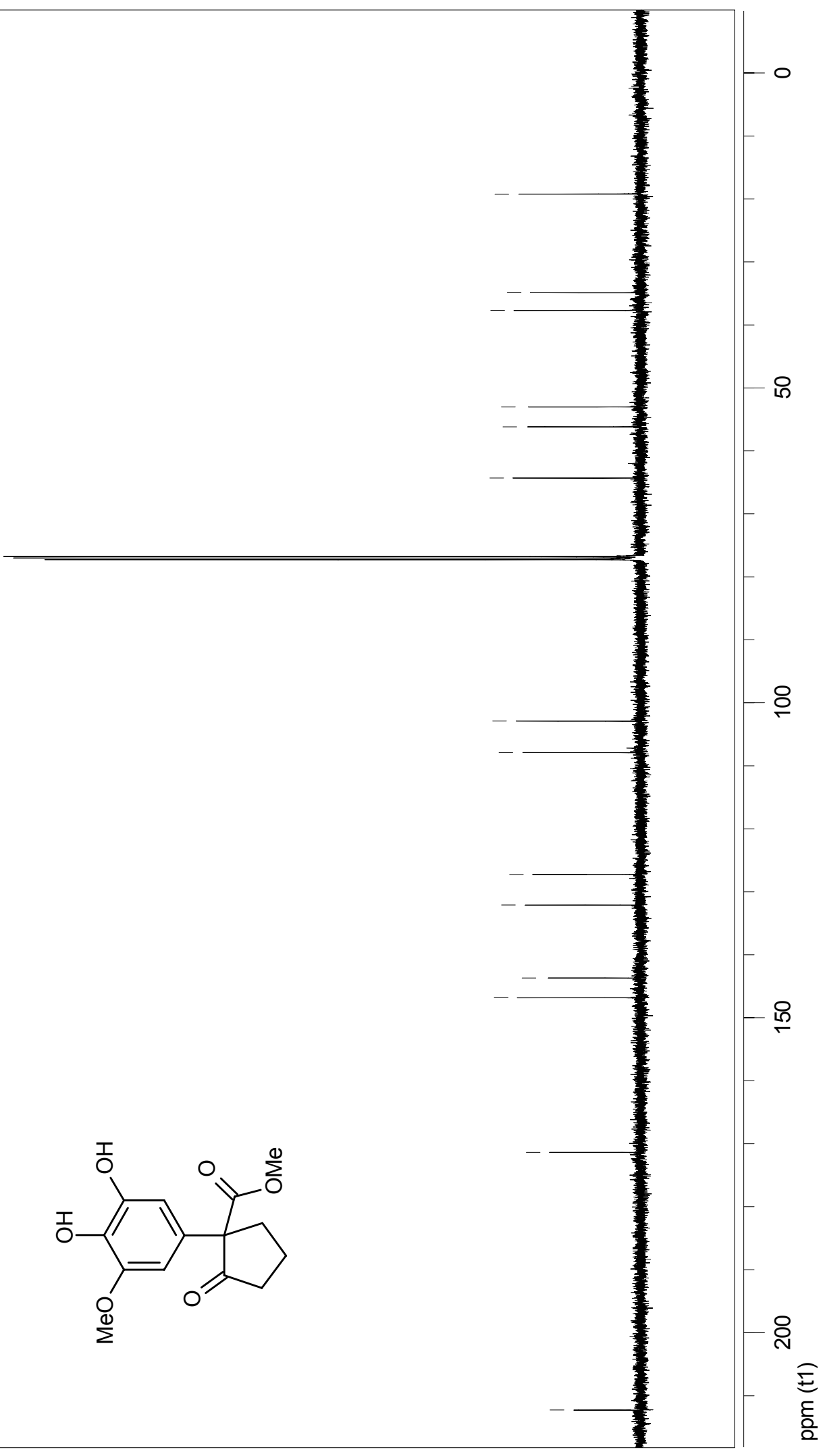


4.9 Spectra for compound $8 \mathrm{i}$

4.9.1 Proton NMR $\left(\mathrm{CDCl}_{3}\right)$

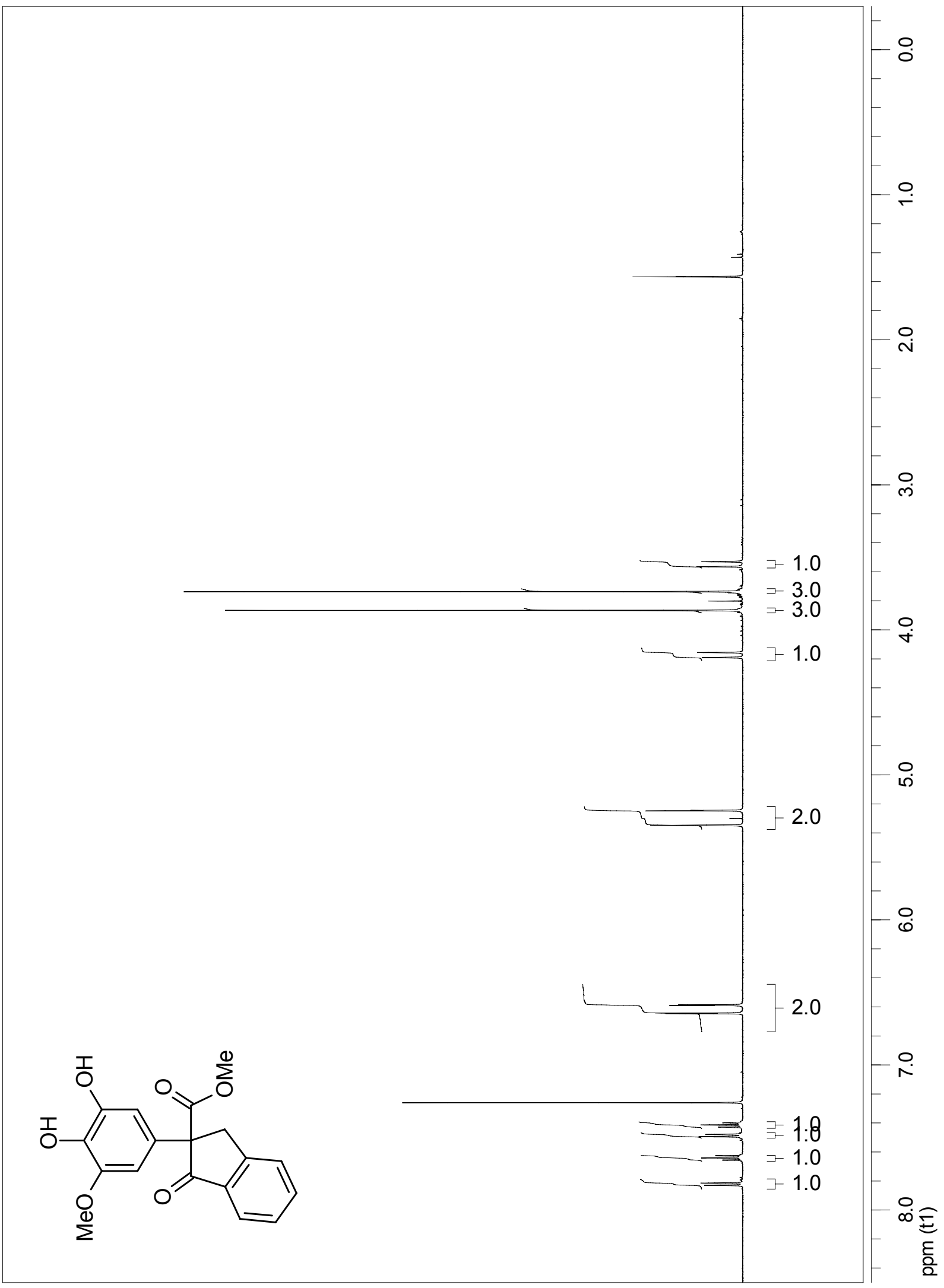


4.9.2 Carbon-13 NMR $\left(\mathrm{CDCl}_{3}\right)$

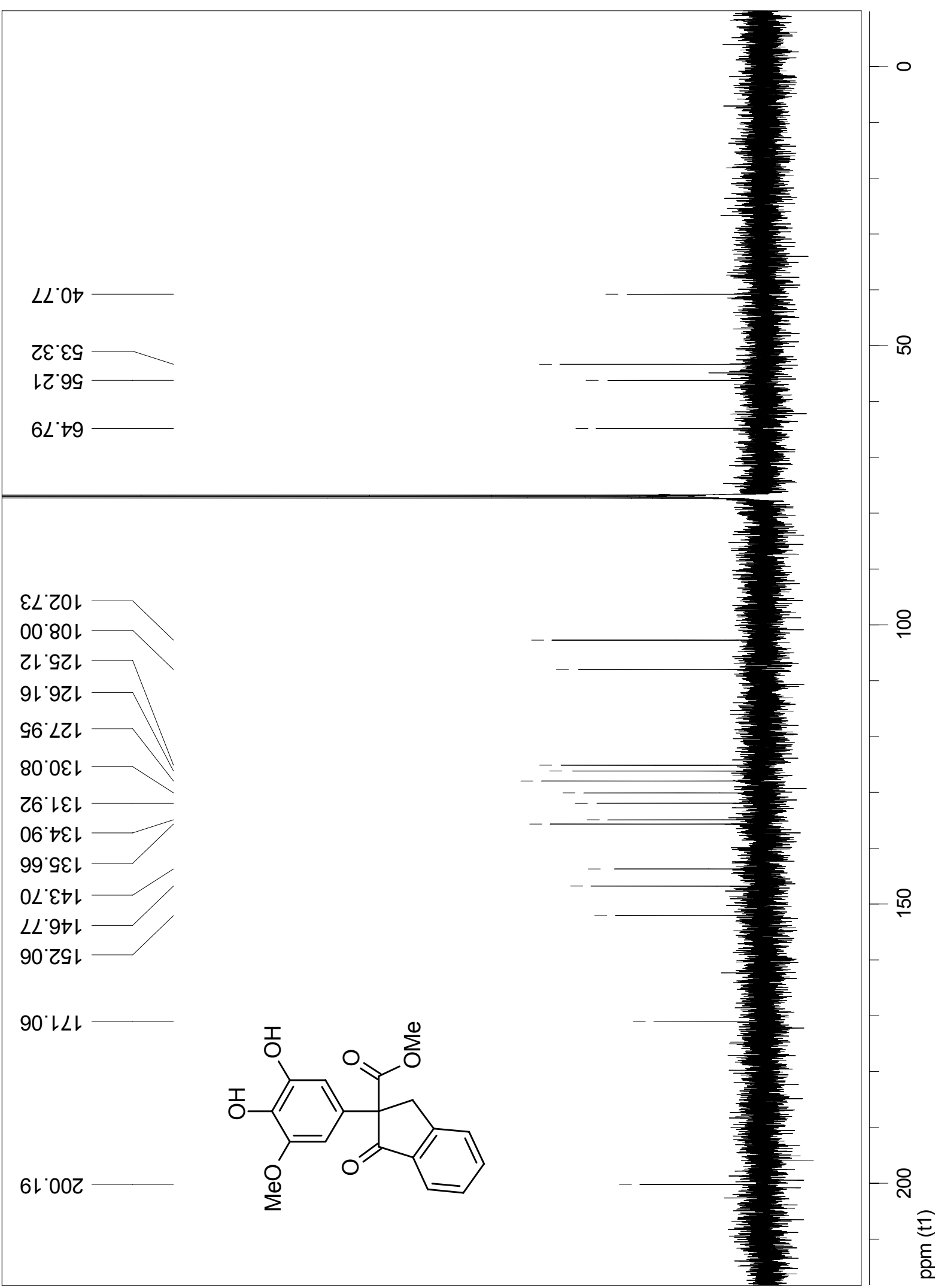


4.10 Spectra for compound 8j

4.10.1 Proton NMR $\left(\mathrm{CDCl}_{3}\right)$

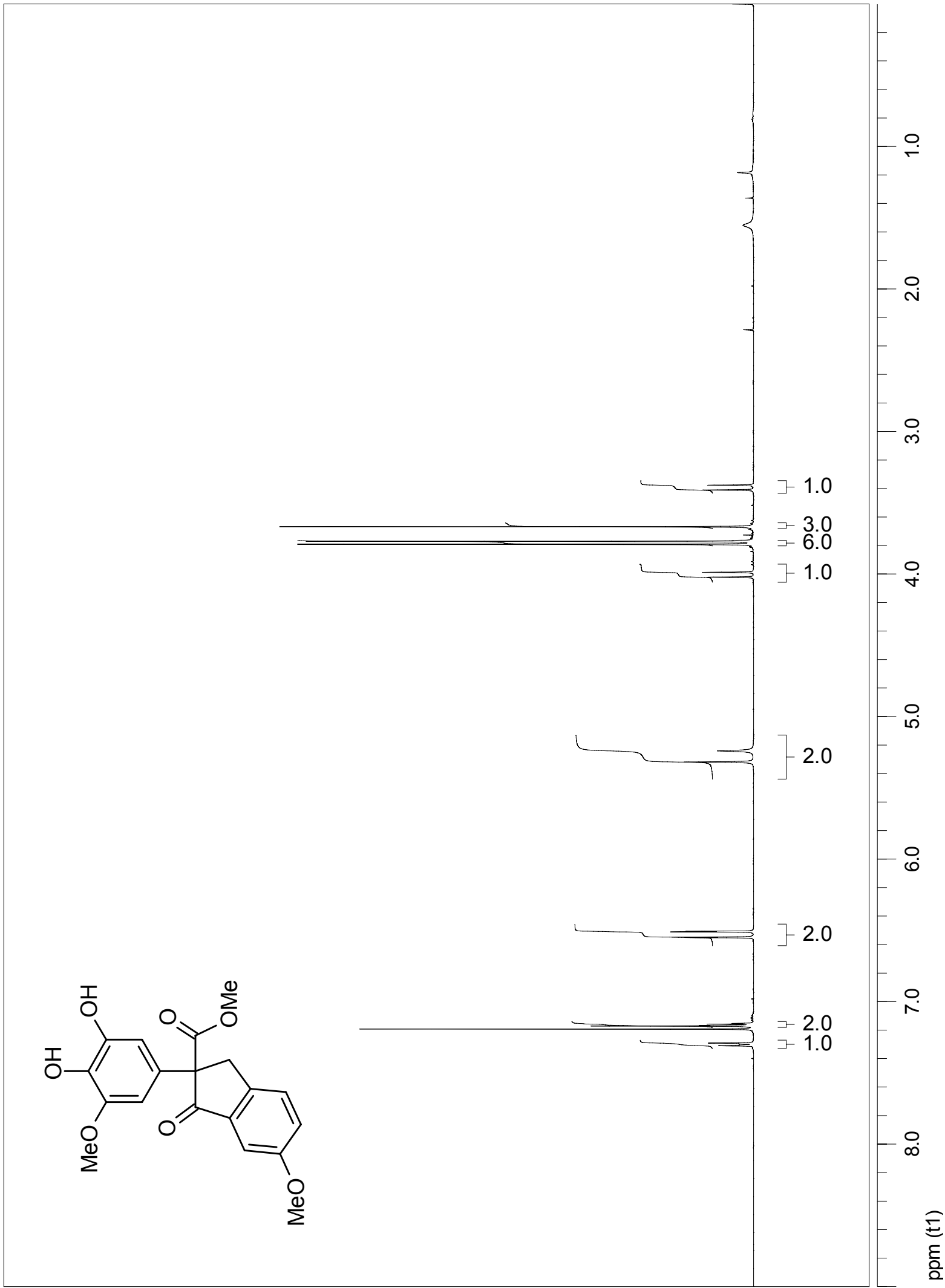




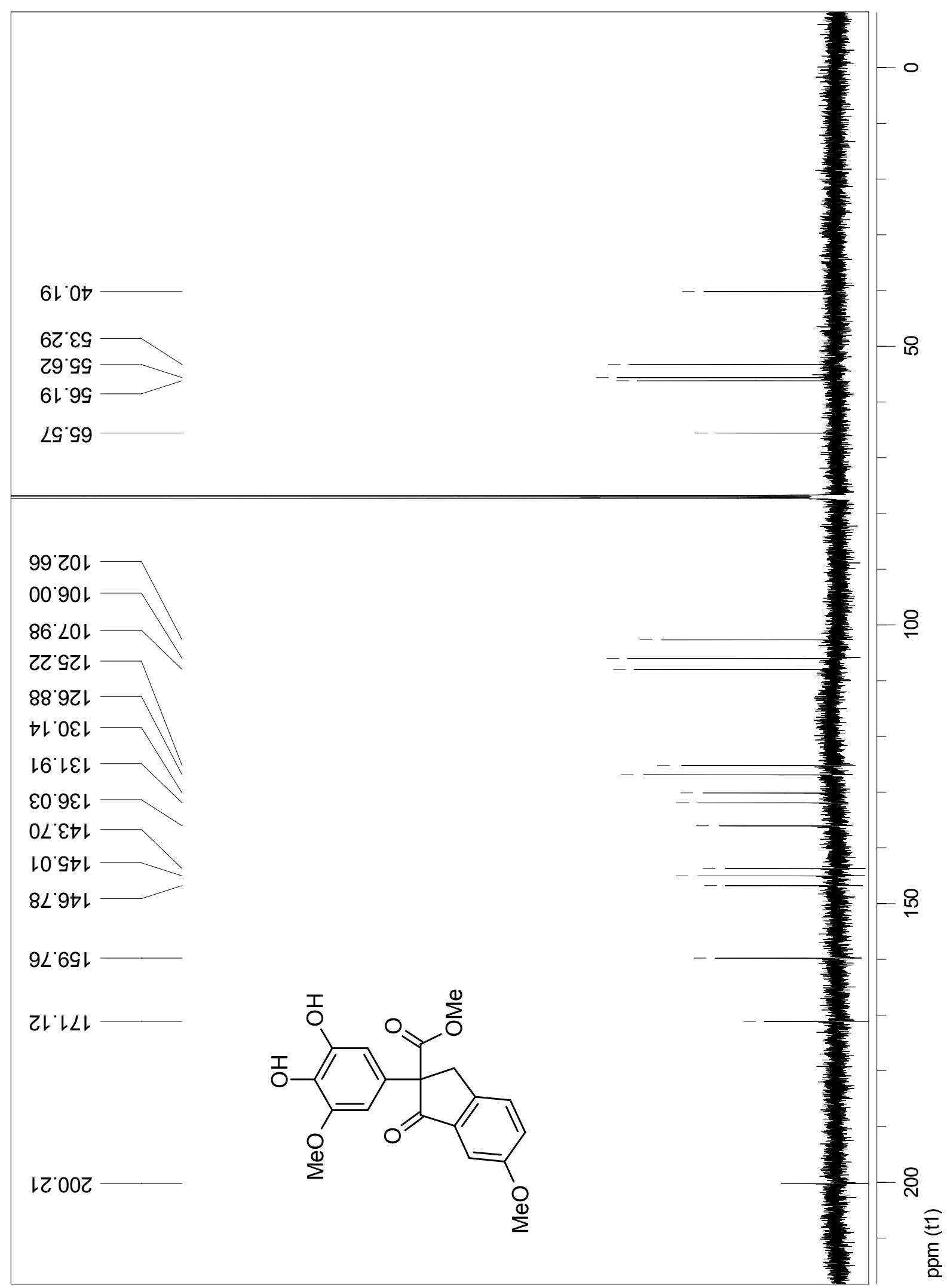


4.11 Spectra for compound 8k

4.11.1 Proton NMR (CDCl$\left.)_{3}\right)$

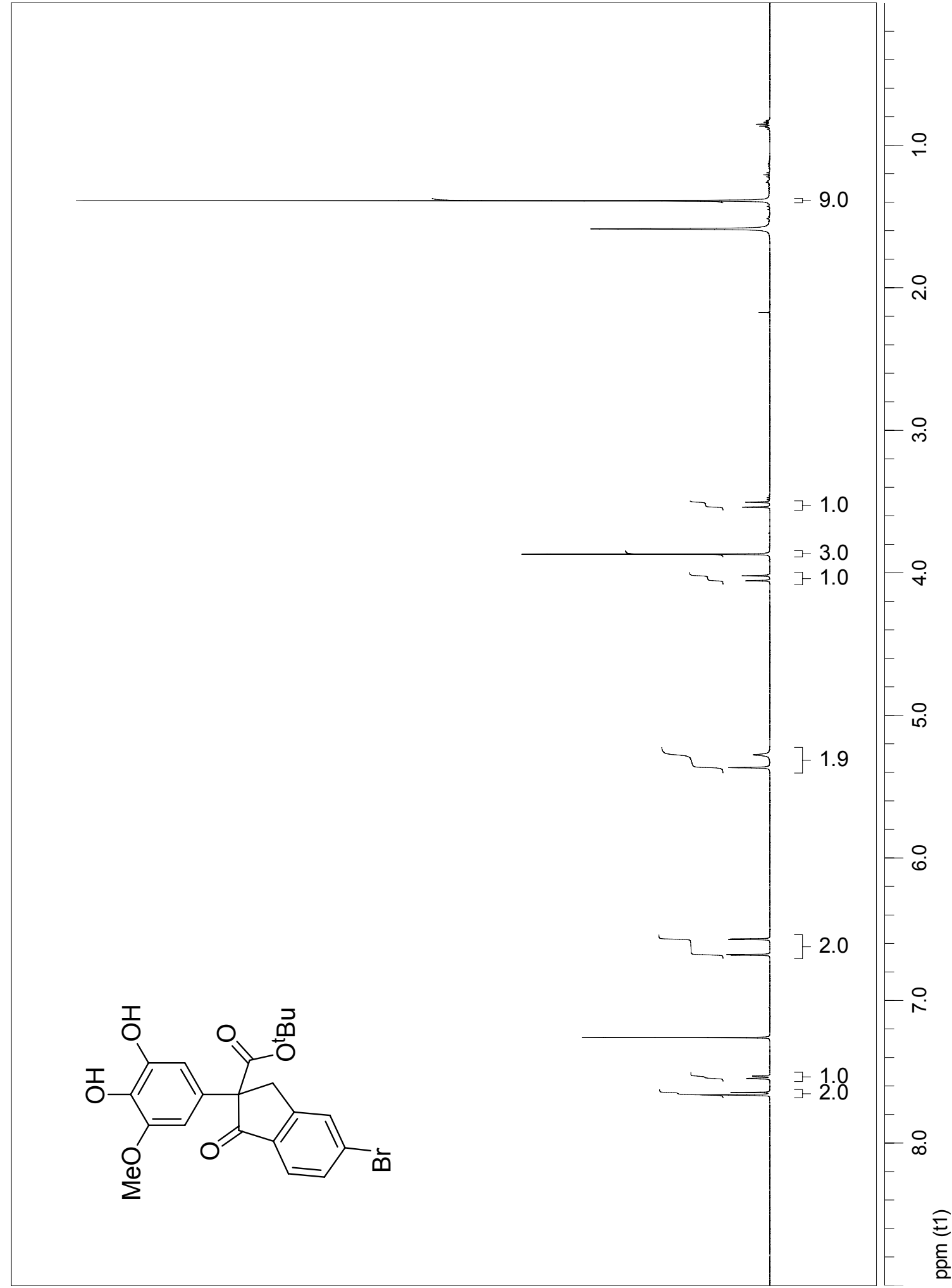




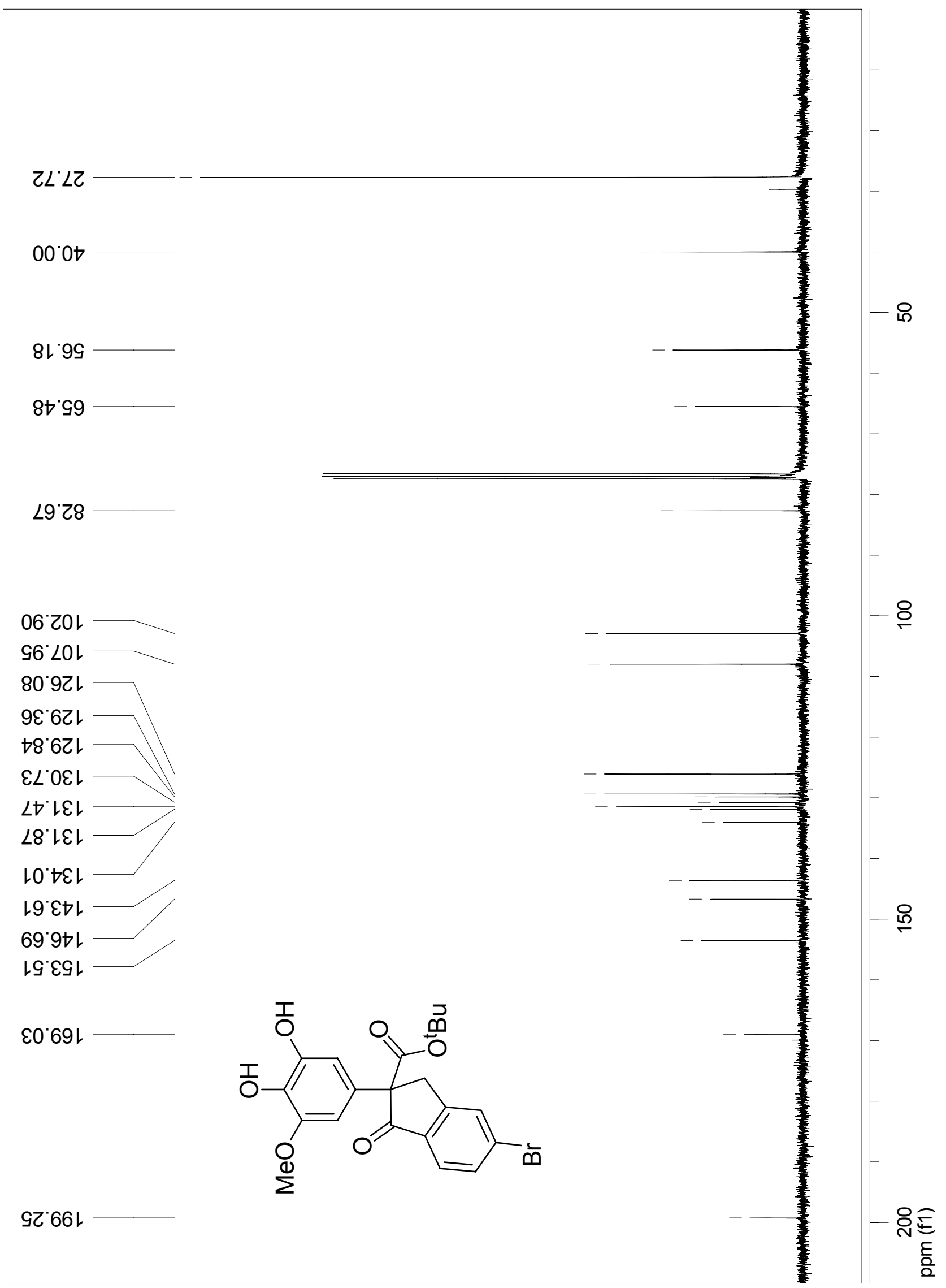


4.12 Spectra for compound 81

4.12.1 Proton NMR $\left(\mathrm{CDCl}_{3}\right)$

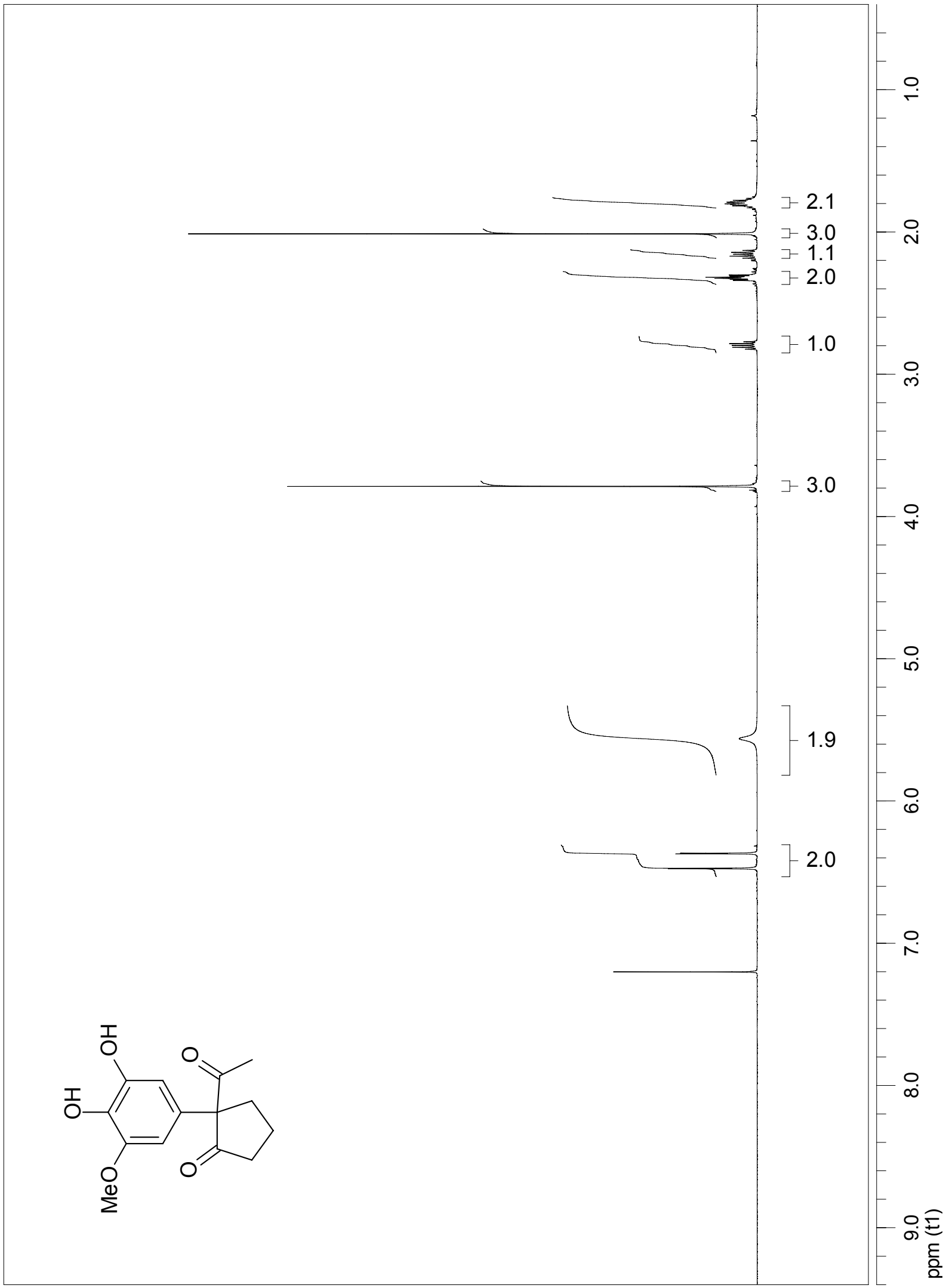


4.12.2 Carbon-13 NMR $\left(\mathrm{CDCl}_{3}\right)$

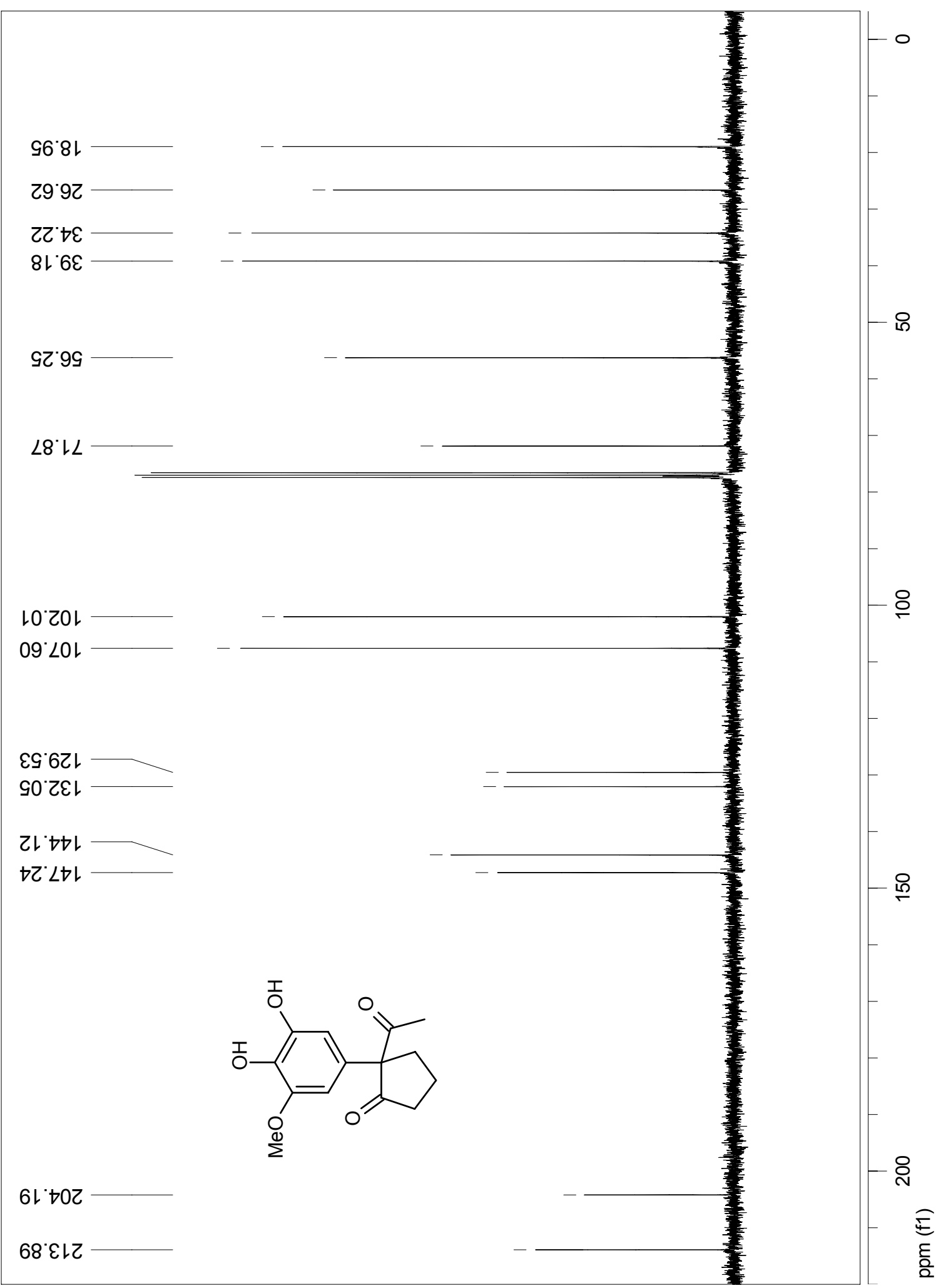




\subsection{Spectra for compound $8 \mathrm{~m}$}

4.13.1 Proton NMR $\left(\mathrm{CDCl}_{3}\right)$

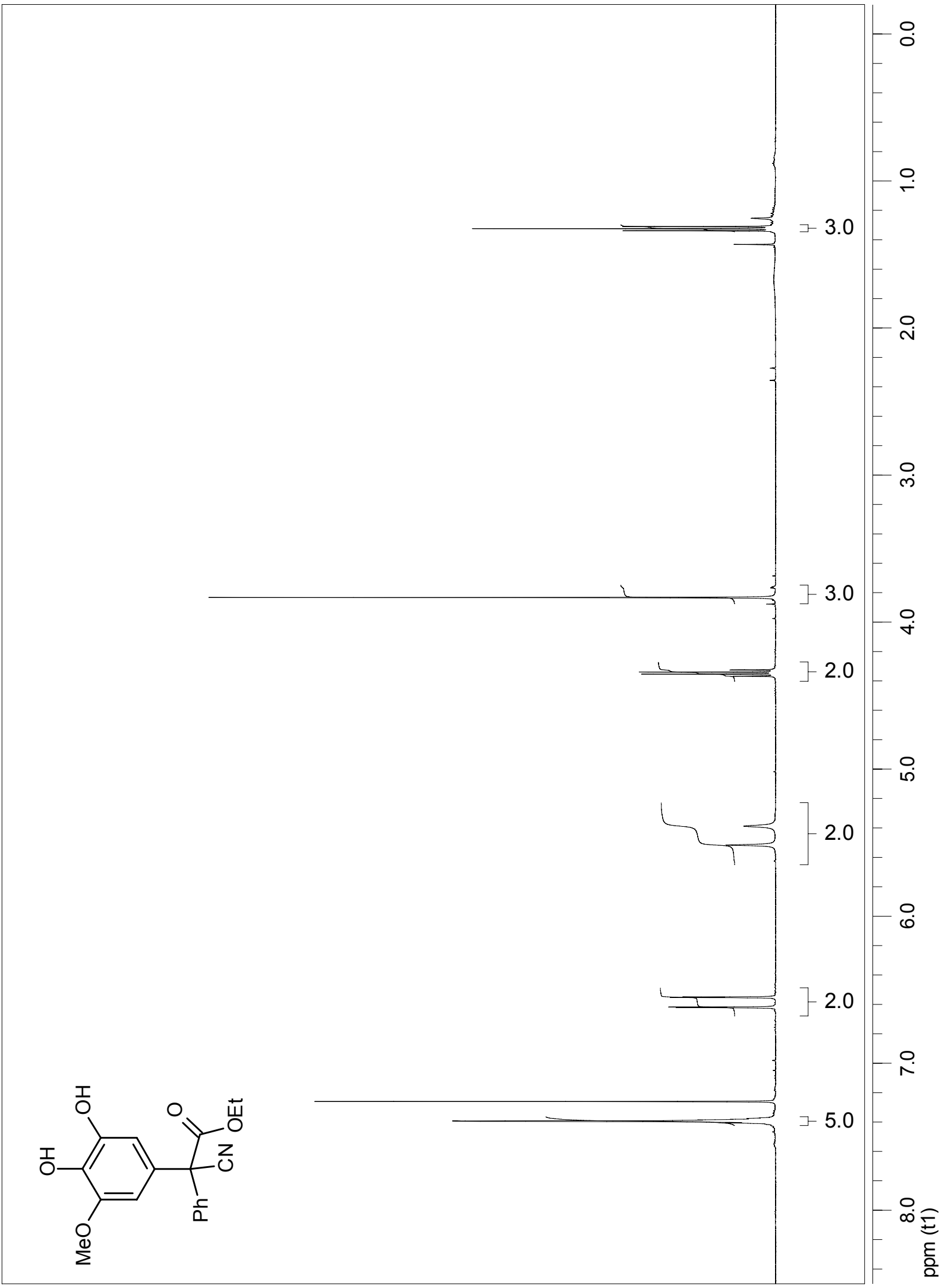


4.13.2 Carbon-13 NMR $\left(\mathrm{CDCl}_{3}\right)$

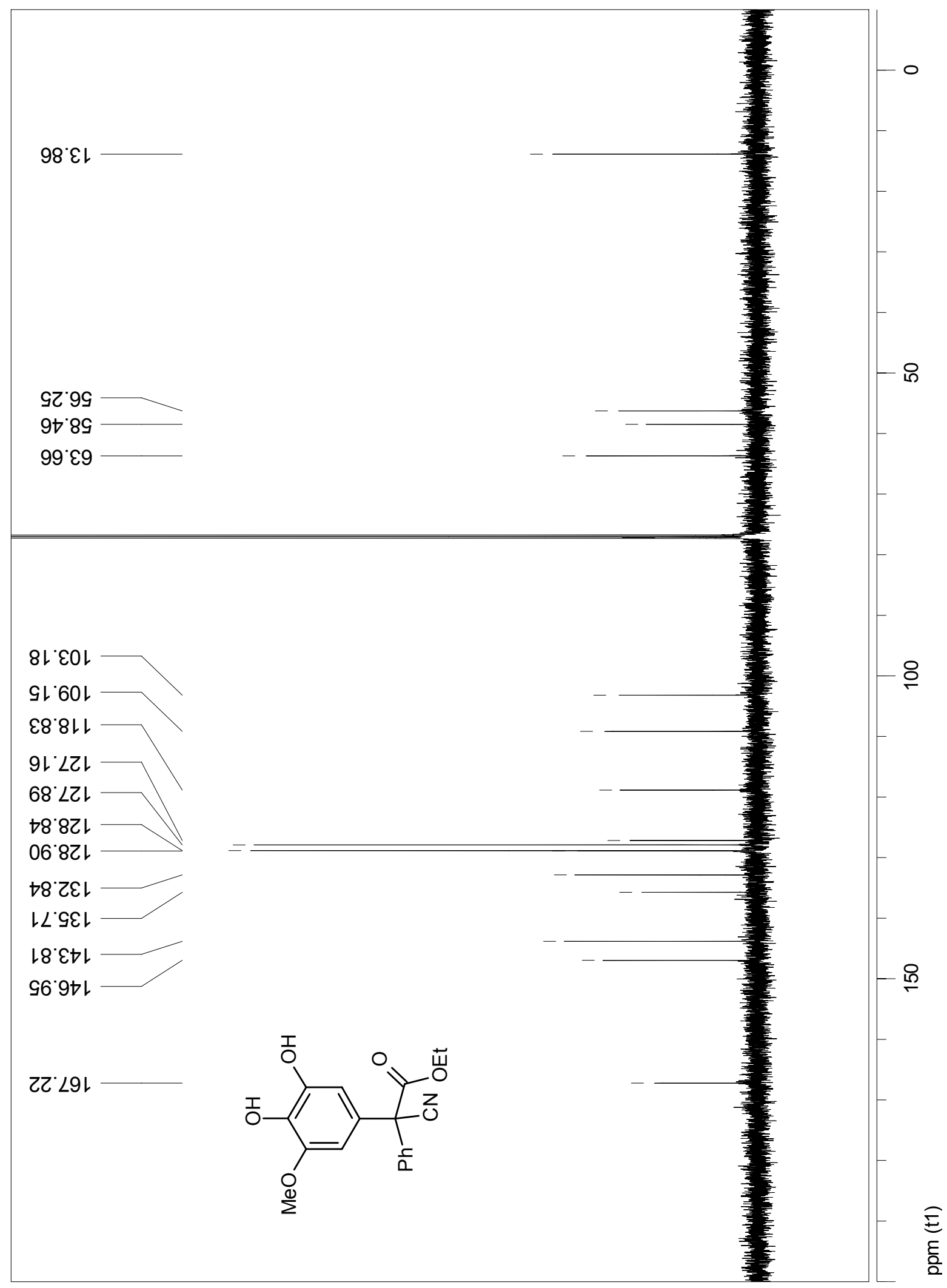


4.14 Spectra for compound 8n

4.14.1 Proton NMR (DMSO)

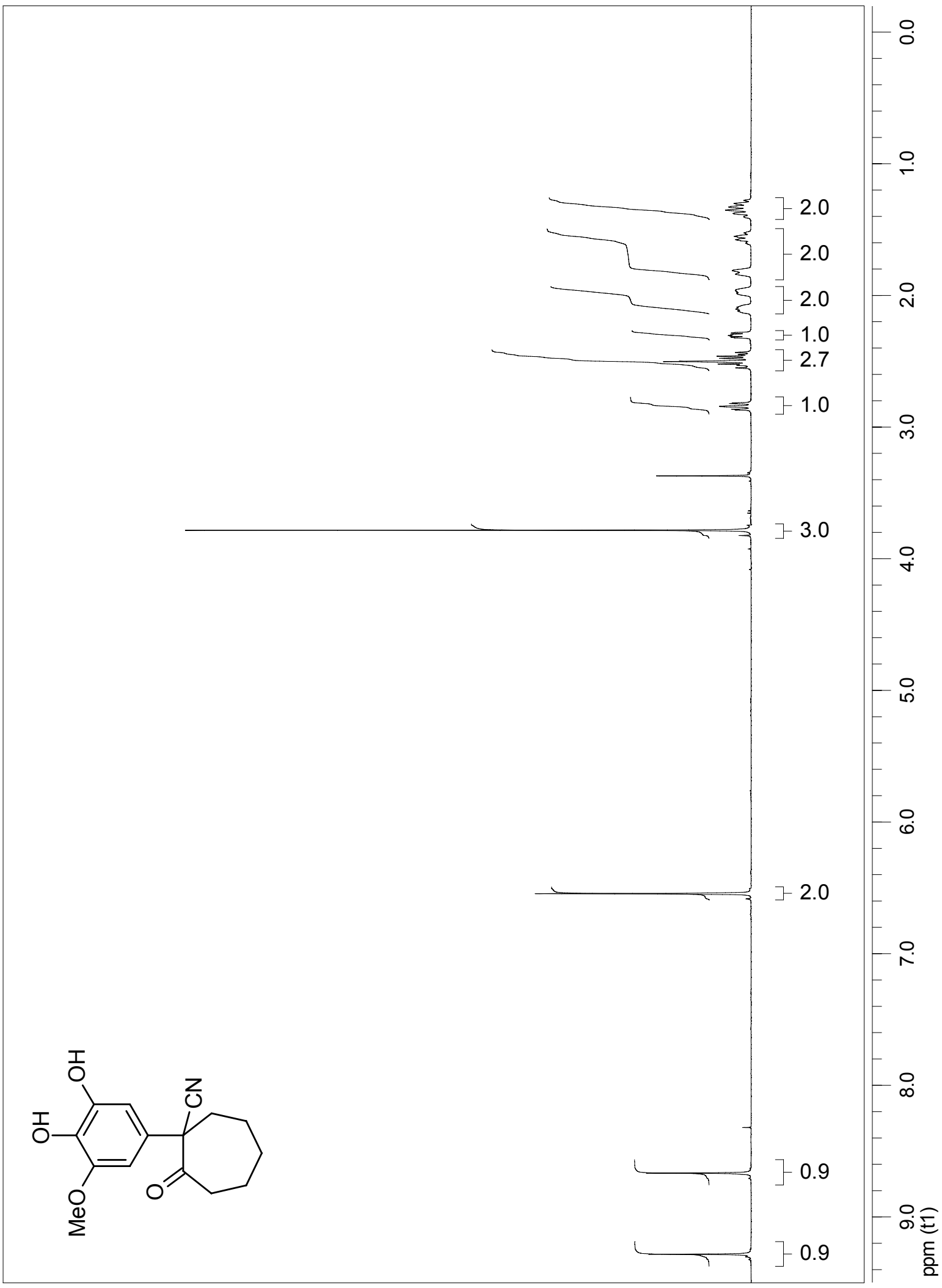


4.14.2 Carbon-13 NMR (DMSO)

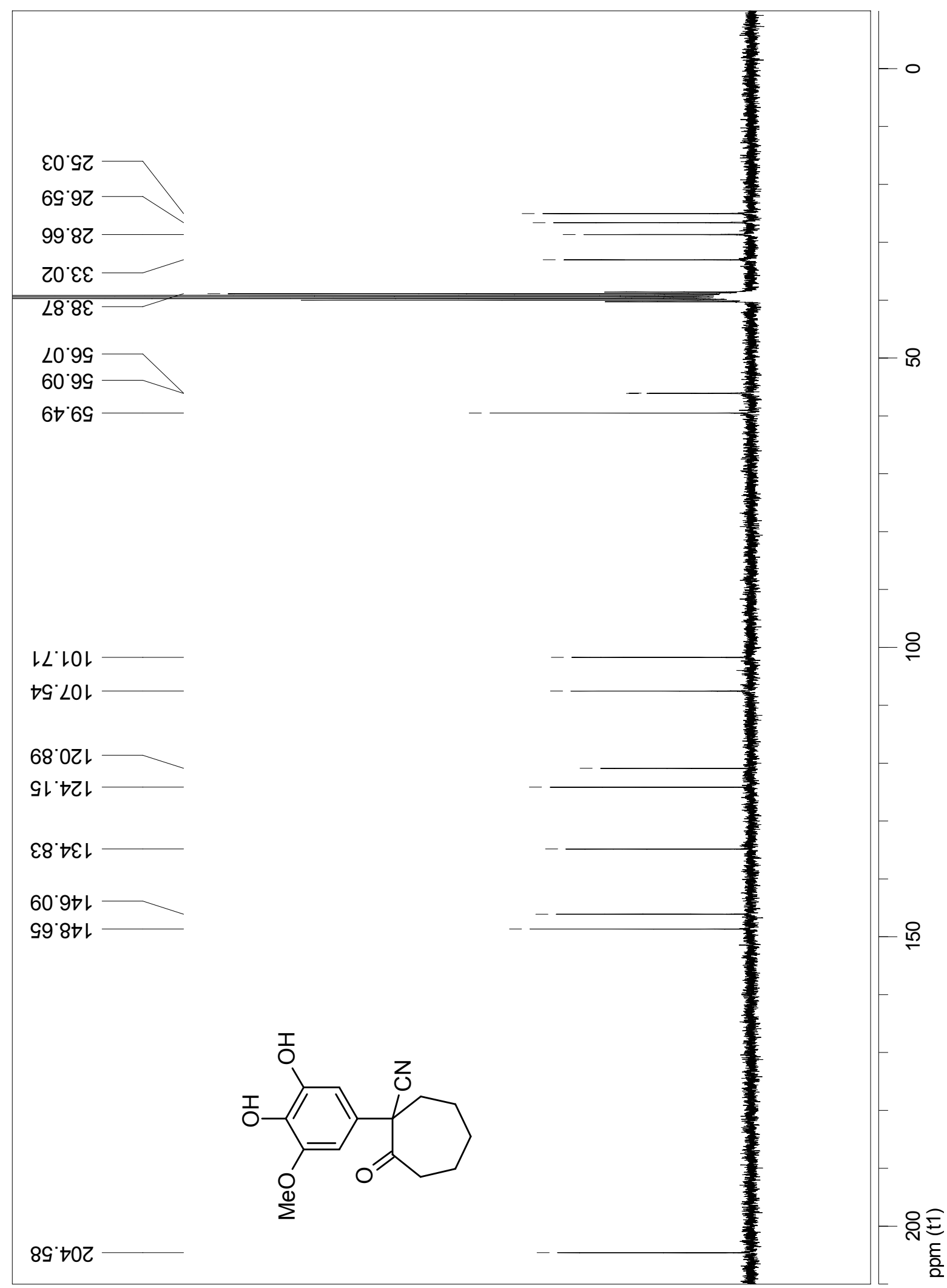


4.15 Spectra for compound 80

4.15.1 Proton NMR $\left(\mathrm{CDCl}_{3}\right)$

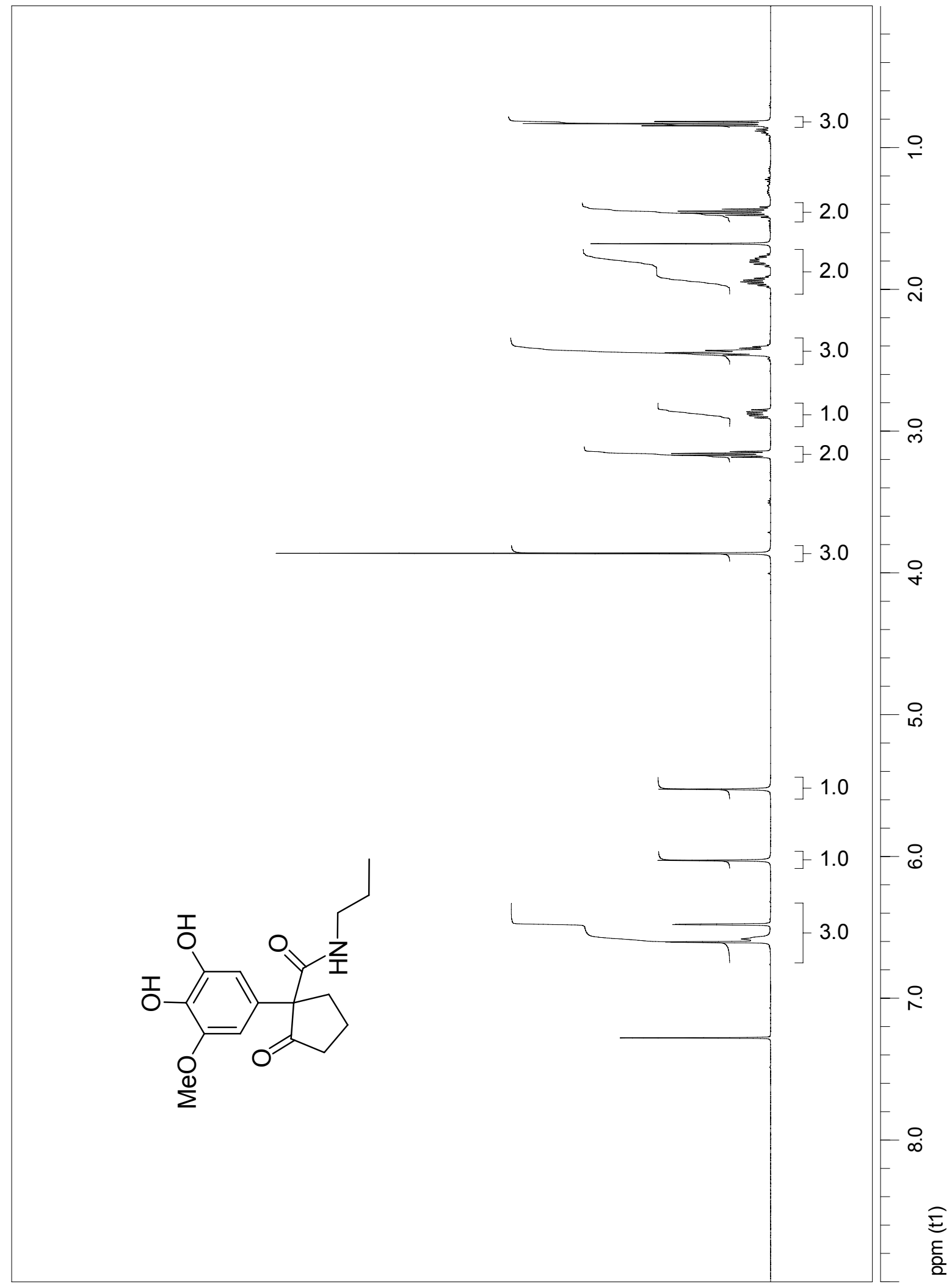


8เレเ

$6 L ' 8 \mathrm{~L}$

$97 \cdot 2 Z$

$08.9 \varepsilon$

LZ'6E

$\varepsilon q^{\prime} \vdash t$

Z२'99

$8 \nabla 79$

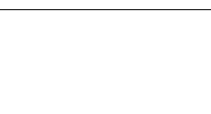

$\varepsilon L \cdot L O$

$6 \varepsilon^{\circ} \angle O L$

$00^{\circ} 0 \varepsilon$

$\downarrow \varepsilon \cdot 乙 \varepsilon\llcorner$

$\forall Z^{\prime} t \nabla L$

$0 \varepsilon \cdot\llcorner t l$

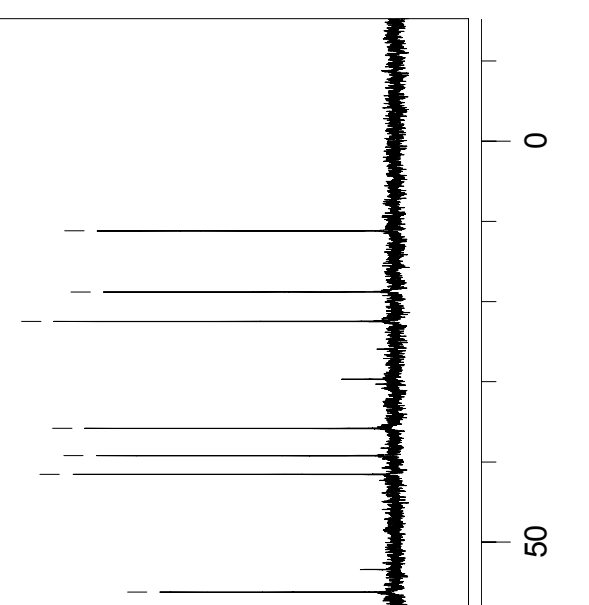

$79^{\circ} 69 \mathrm{l}$

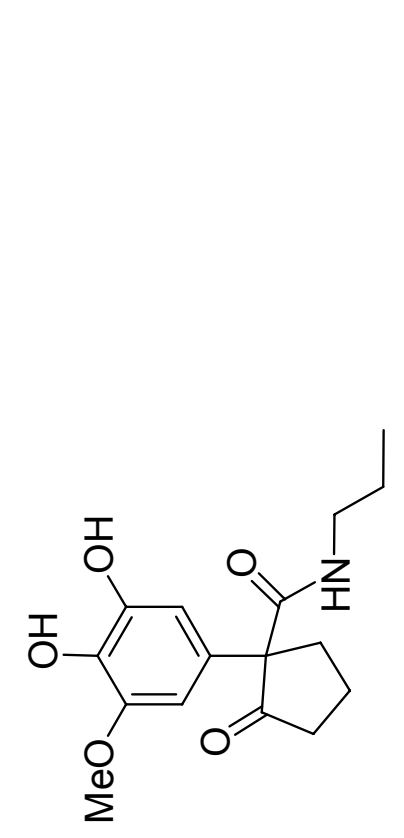

$98: \angle L Z$ 
4.16 Spectra for compound 8p

4.16.1 Proton NMR

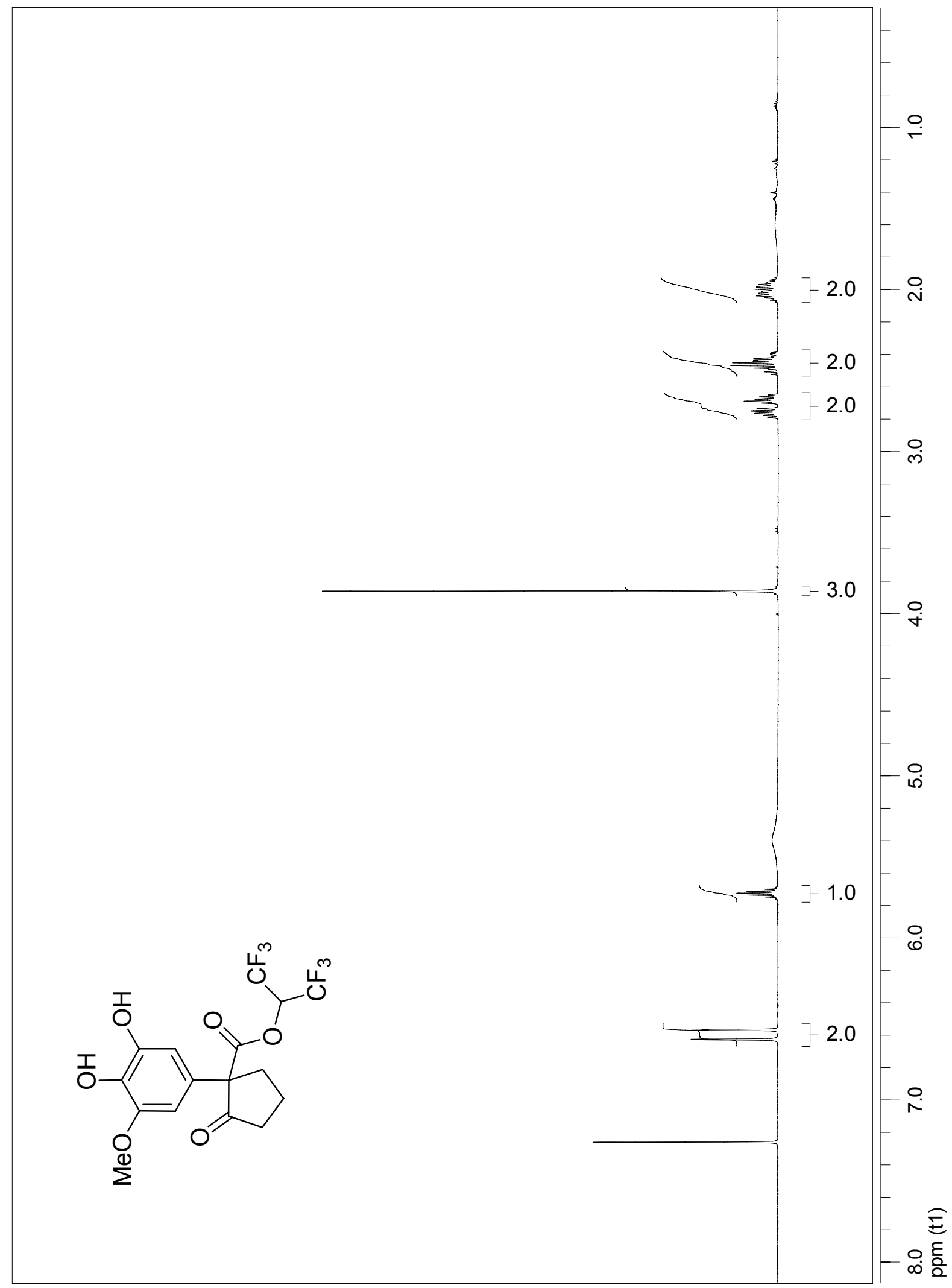


4.16.2 Carbon-13 NMR $\left(\mathrm{CDCl}_{3}\right)$

$\angle L 6 L$

$0\llcorner\nabla \varepsilon$

$\varepsilon \mathcal{C}^{\prime}\llcorner\mathcal{E}$

$02 \cdot 99$

乙9'๕9

$\angle 0^{\circ} \angle 9$

ㄱ'ㅇㄴ

$9 L \angle O L$

$60^{\circ} 6$ L

乙ย'เ乙レ

$600^{\circ} \mathrm{LL}$

GG'ZEᄂ

$\angle 8^{\circ} \varepsilon \triangleright \downarrow$

$20^{\circ} \angle \nabla L$

앙ㄴ

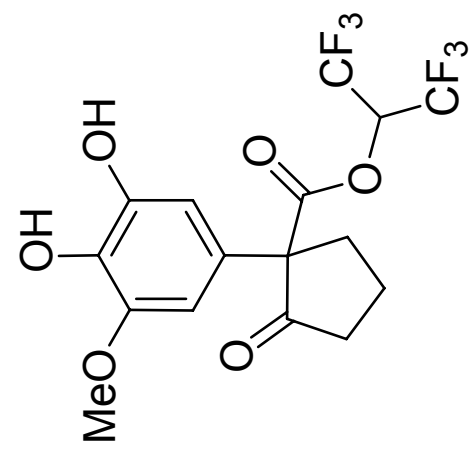

$\frac{E}{E}$ 
4.17 Spectra for compound 11

4.17.1 Proton NMR $\left(\mathrm{CDCl}_{3}\right)$

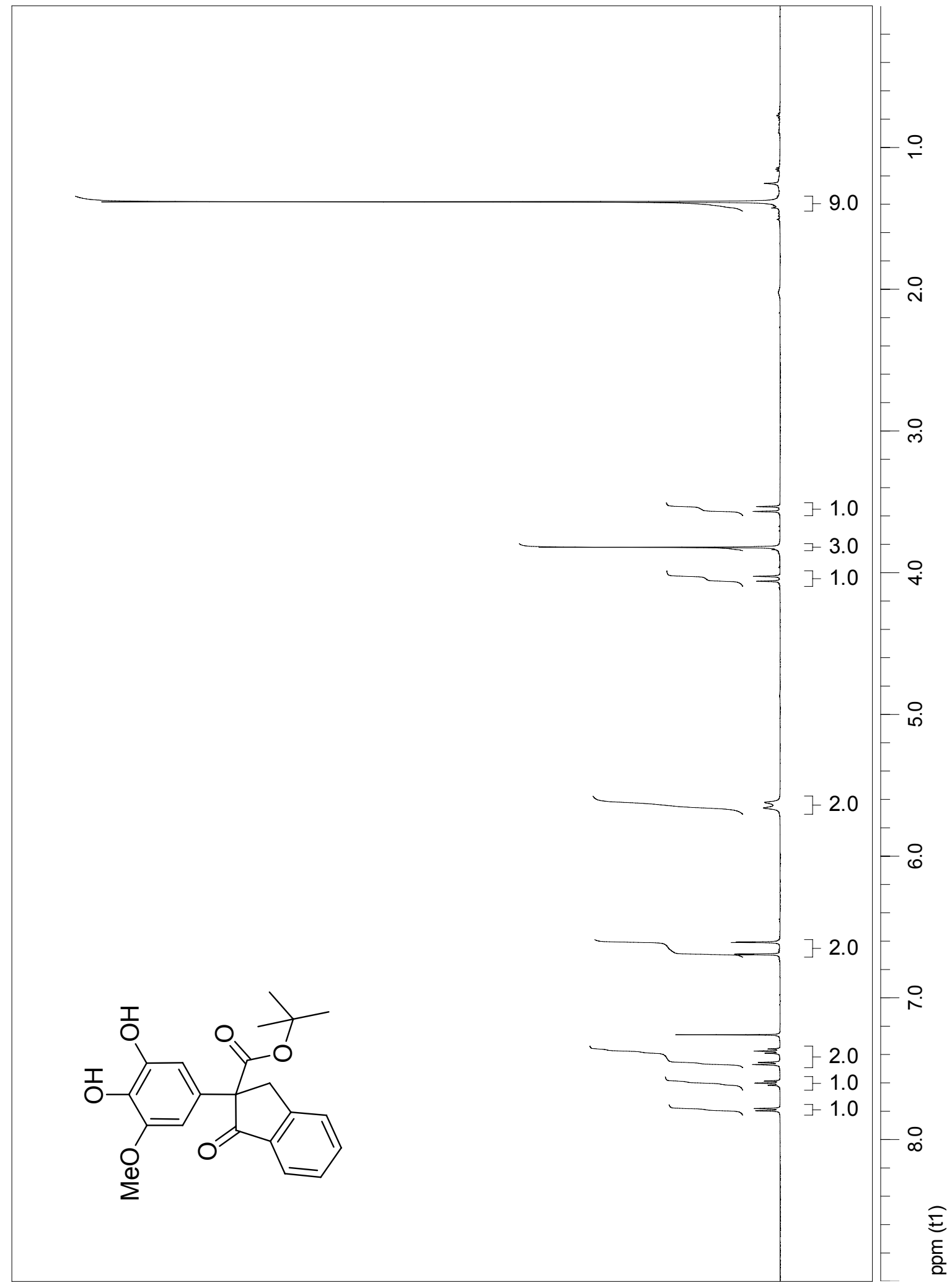


4.17.2 Carbon-13 NMR $\left(\mathrm{CDCl}_{3}\right)$

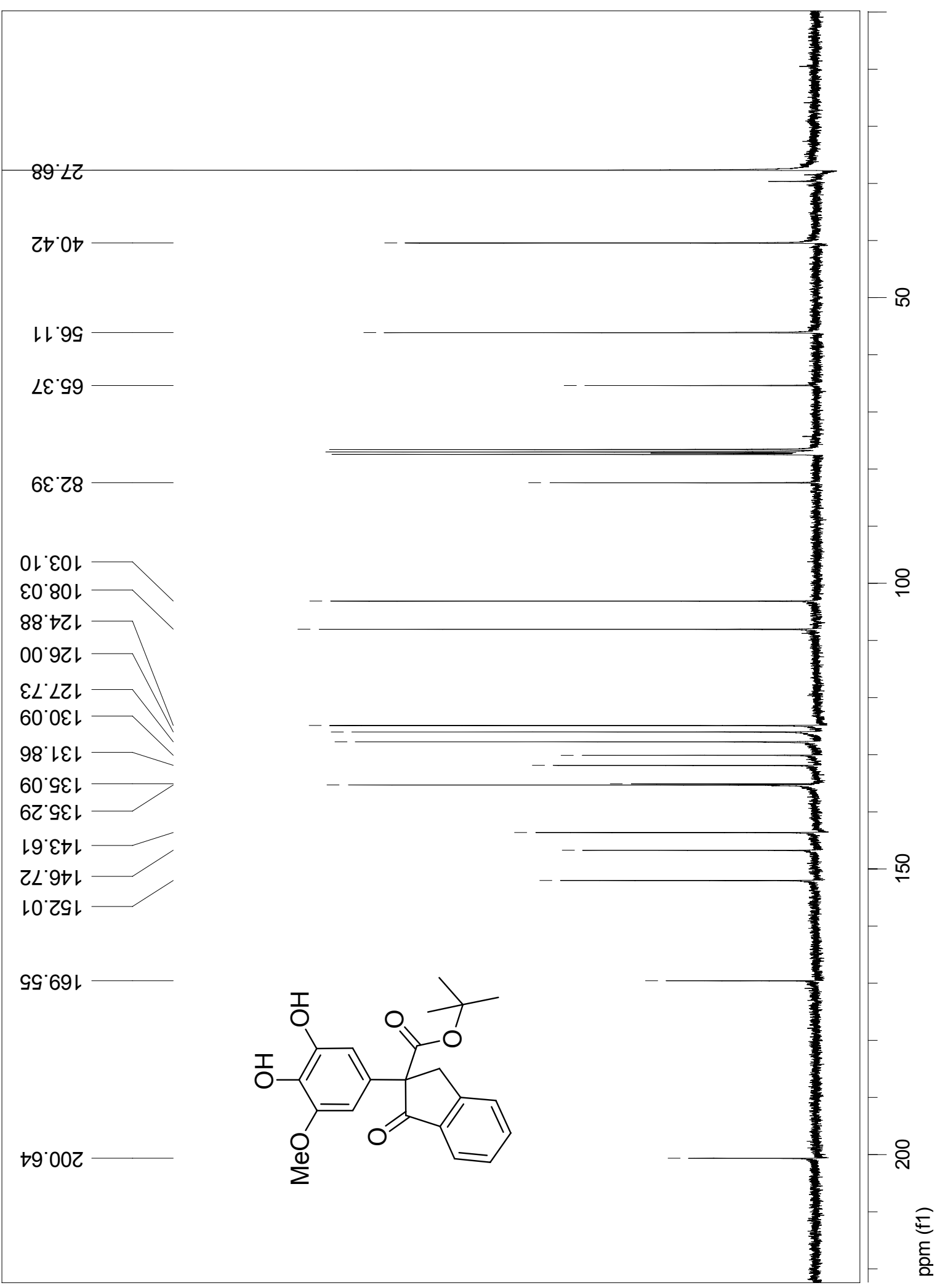


4.17.3 HPLC trace of compound 11 - racemate synthesised with ps-BEMP

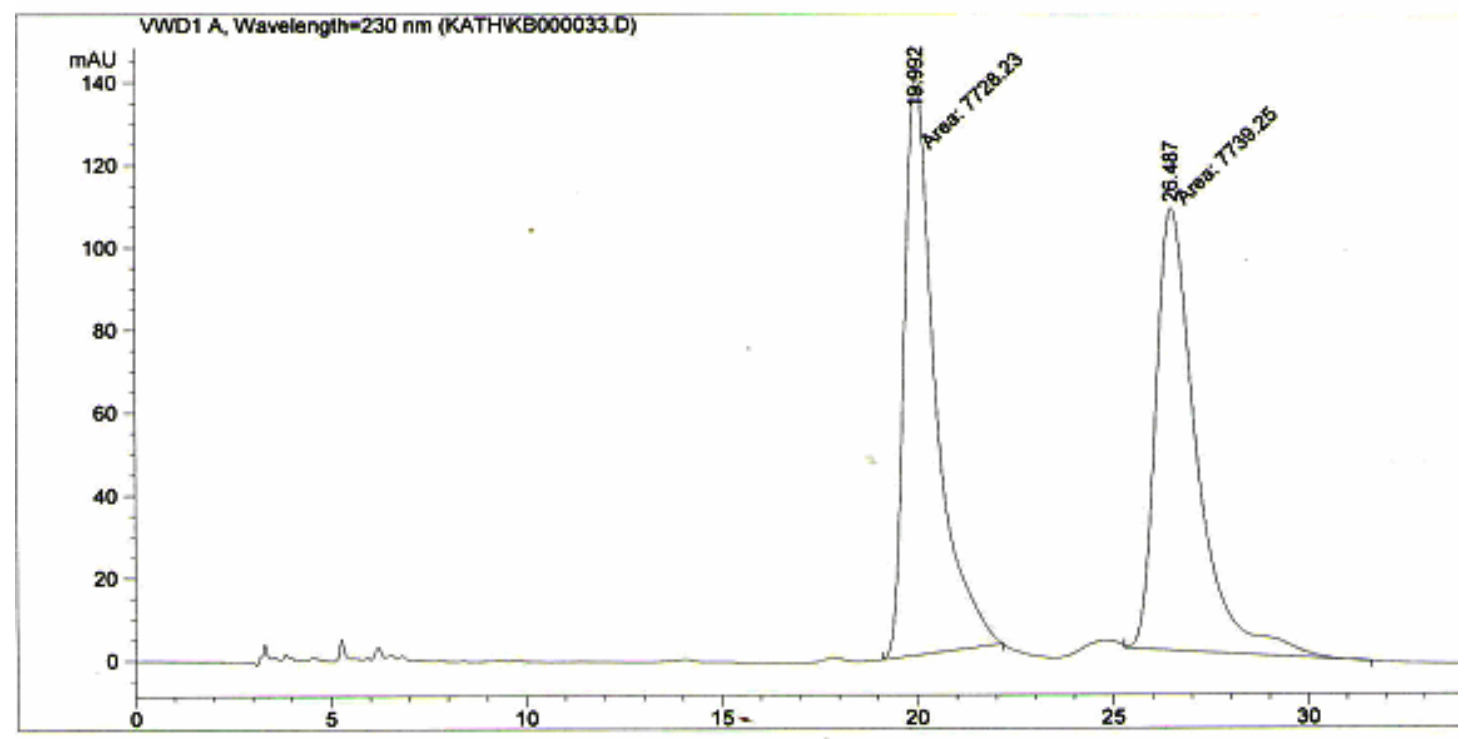

Area Percent Report

$\begin{array}{lll}\text { Sorted By } & : & \text { Signal } \\ \text { Multiplier } & : & 1.0000 \\ \text { Dilution } & : & 1.0000\end{array}$

Use Multiplier \& Dilution Factor with ISTDs

Signal 1: VWD1 A, Wavelength-230 nm

\begin{tabular}{|c|c|c|c|c|c|c|}
\hline $\begin{array}{c}\text { Peak } \\
*\end{array}$ & $\begin{array}{c}\text { RetTime } \\
\text { [min] }\end{array}$ & Type & $\begin{array}{l}\text { Width } \\
\text { [min] }\end{array}$ & $\begin{array}{c}\text { Area } \\
{\left[m A U^{*} s\right]}\end{array}$ & $\begin{array}{l}\text { Height } \\
\text { [mAU] }\end{array}$ & $\begin{array}{c}\text { Area } \\
\text { \& }\end{array}$ \\
\hline $\begin{array}{l}1 \\
2\end{array}$ & $\begin{array}{l}19.992 \\
26.487\end{array}$ & $\begin{array}{l}M \\
M M\end{array}$ & $\begin{array}{l}0.9184 \\
1.2053\end{array}$ & $\begin{array}{l}7728.22607 \\
7739.25049\end{array}$ & $\begin{array}{l}140.24998 \\
107.01434\end{array}$ & $\begin{array}{l}49.9644 \\
50.0356\end{array}$ \\
\hline Total & : & & & $1.54675 \mathrm{e} 4$ & 247.26433 & \\
\hline
\end{tabular}


4.17.4 HPLC trace for compound 11, synthesised with catalyst 12

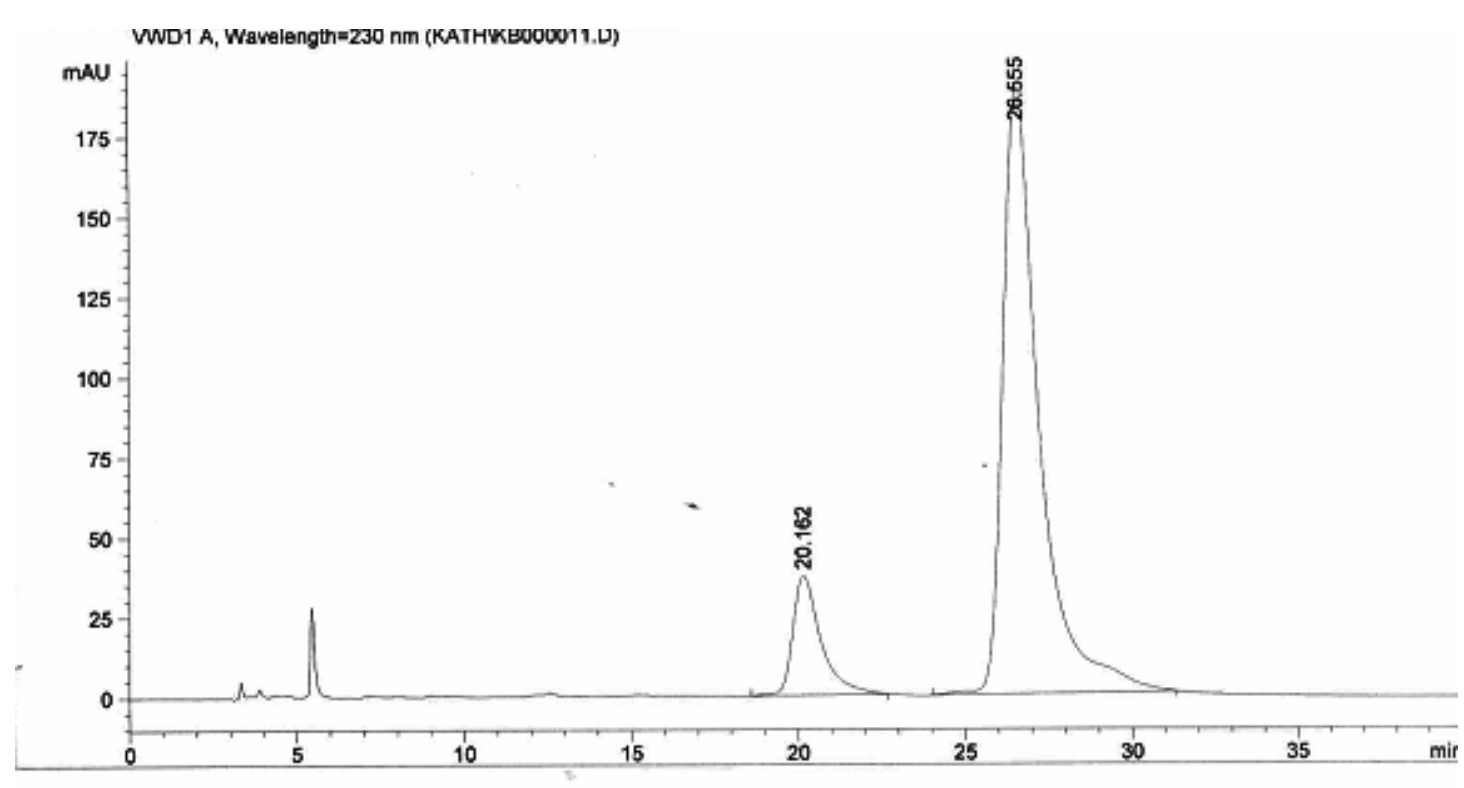

Area Percent Report

$\begin{array}{lll}\text { Sorted By } & : & \text { Signal } \\ \text { Multiplier } & : & 1.0000\end{array}$

$\begin{array}{lll}\text { Multiplier } & : & 1.0000 \\ \text { Dilution } & : & 1.0000\end{array}$

Use Multiplier \& Dilution Factor with ISTDs

Signal 1: VWD1 A, Wavelength $=230 \mathrm{~nm}$

\begin{tabular}{|c|c|c|c|c|c|c|}
\hline $\begin{array}{c}\text { Peak } \\
\neq\end{array}$ & $\begin{array}{c}\text { RetTime } \\
\text { [min] }\end{array}$ & Type & $\begin{array}{l}\text { Width } \\
\text { [min] }\end{array}$ & $\begin{array}{c}\text { Area } \\
\text { [mAU*s] }\end{array}$ & $\begin{array}{l}\text { Helght } \\
\text { [MAU] }\end{array}$ & $\begin{array}{c}\text { Area } \\
8\end{array}$ \\
\hline $\begin{array}{l}1 \\
2\end{array}$ & $\begin{array}{l}20.162 \\
26.555\end{array}$ & $\begin{array}{l}\mathrm{BB} \\
\mathrm{BB}\end{array}$ & $\begin{array}{l}0.8276 \\
1.0992\end{array}$ & $\begin{array}{l}2189.92334 \\
1.44009 e 4\end{array}$ & $\begin{array}{r}37.32101 \\
189.36653\end{array}$ & $\begin{array}{l}13.1996 \\
86.8004\end{array}$ \\
\hline Tota & : & & & $1.6590 \mathrm{Be} 4$ & 226.68754 & \\
\hline
\end{tabular}


4.17.5 HPLC trace for compound 11, synthesised using catalyst 13

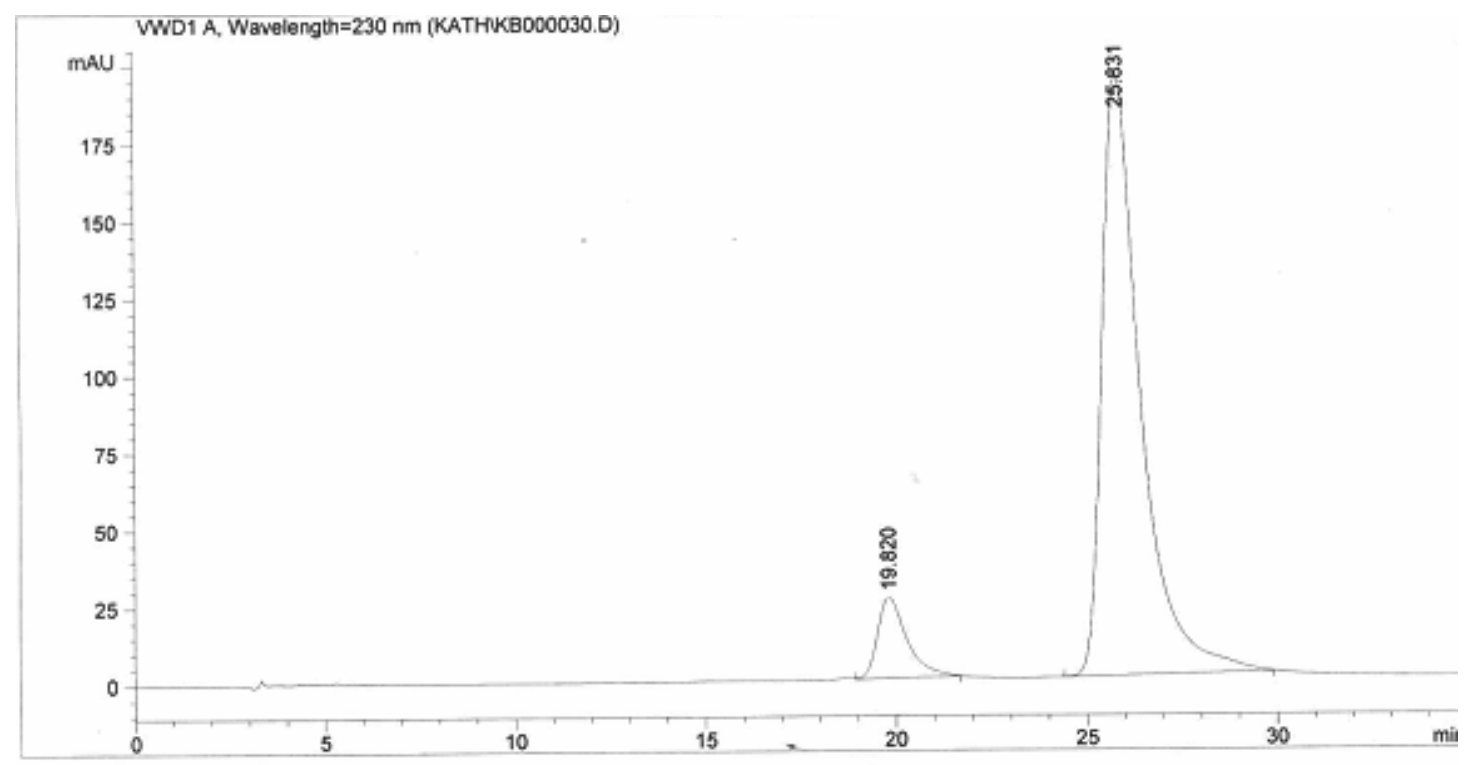

Area Percent Report

$\begin{array}{lll}\text { Sorted By } & : & \text { Signal } \\ \text { Multiplier } & : & 1.0000\end{array}$

$\begin{array}{lll}\text { Multiplier } & : & 1.0000 \\ \text { Dilution } & : & 1.0000\end{array}$

Use Multiplier \& Dilution Factor with ISTDs

Signal 1: VWD1 A, Wavelength-230 nm

\begin{tabular}{|c|c|c|c|c|c|c|}
\hline $\begin{array}{c}\text { Peak } \\
\#\end{array}$ & $\begin{array}{l}\text { RetTime } \\
\text { [min] }\end{array}$ & Type & $\begin{array}{l}\text { Width } \\
\text { [min] }\end{array}$ & $\begin{array}{c}\text { Area } \\
{\left[\mathrm{mAO}^{*} \mathrm{~s}\right]}\end{array}$ & $\begin{array}{l}\text { Height } \\
\text { [mAu] }\end{array}$ & $\underset{8}{\text { Area }}$ \\
\hline $\begin{array}{l}1 \\
2\end{array}$ & $\begin{array}{l}19.820 \\
25.831\end{array}$ & $\begin{array}{l}\text { VB } \\
\text { VB }\end{array}$ & $\begin{array}{l}0.6285 \\
0.9210\end{array}$ & $\begin{array}{l}1399.94080 \\
1.36281 e 4\end{array}$ & $\begin{array}{r}26.32172 \\
194.12379\end{array}$ & $\begin{array}{r}9.3155 \\
90.6845\end{array}$ \\
\hline
\end{tabular}


4.18 Spectra for catalyst 13

4.18.1 Proton NMR (MeOD) of catalyst 13

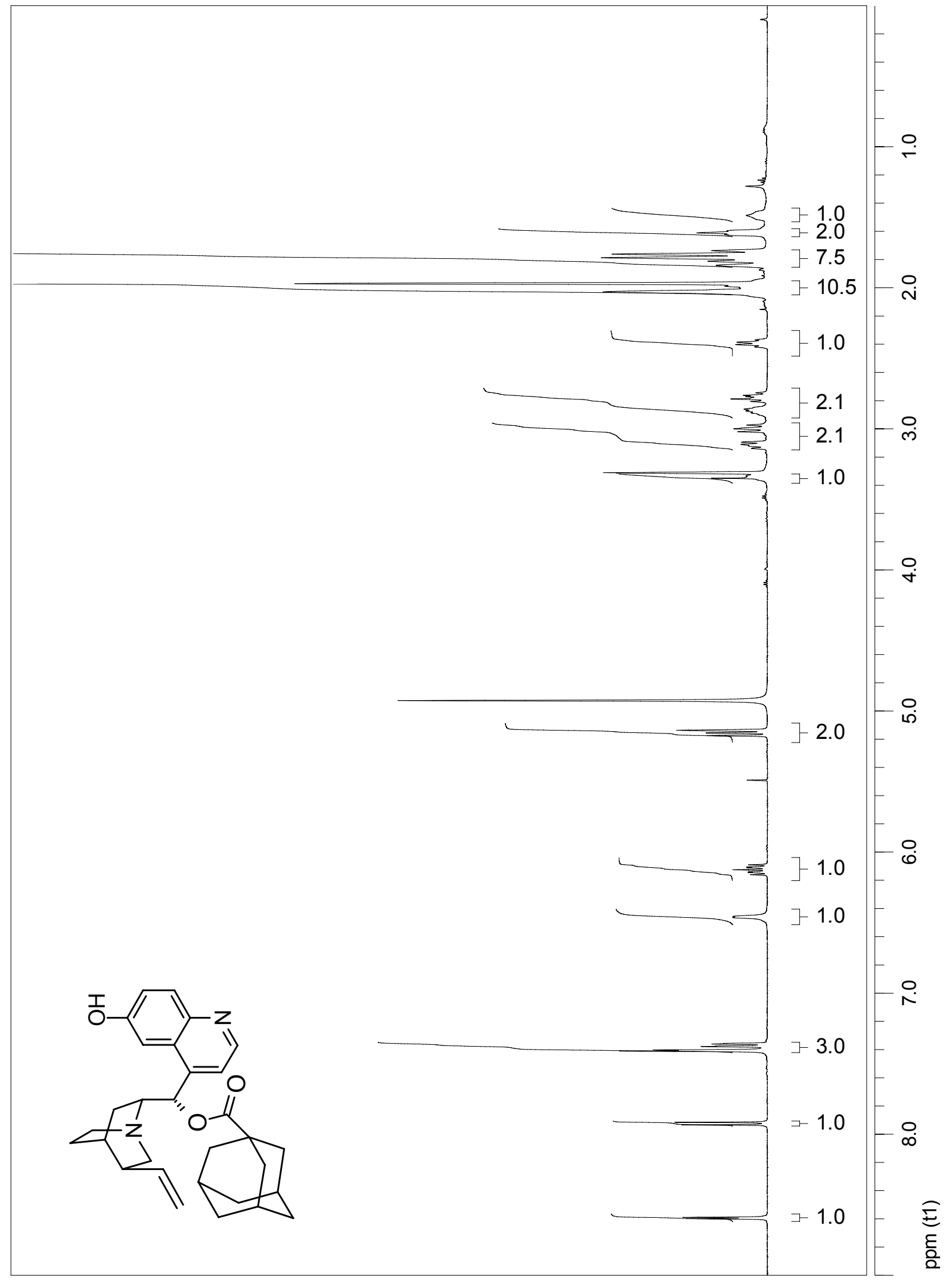


4.18.2 Carbon-13 NMR $\left(\mathrm{CDCl}_{3}\right)$ of catalyst 13

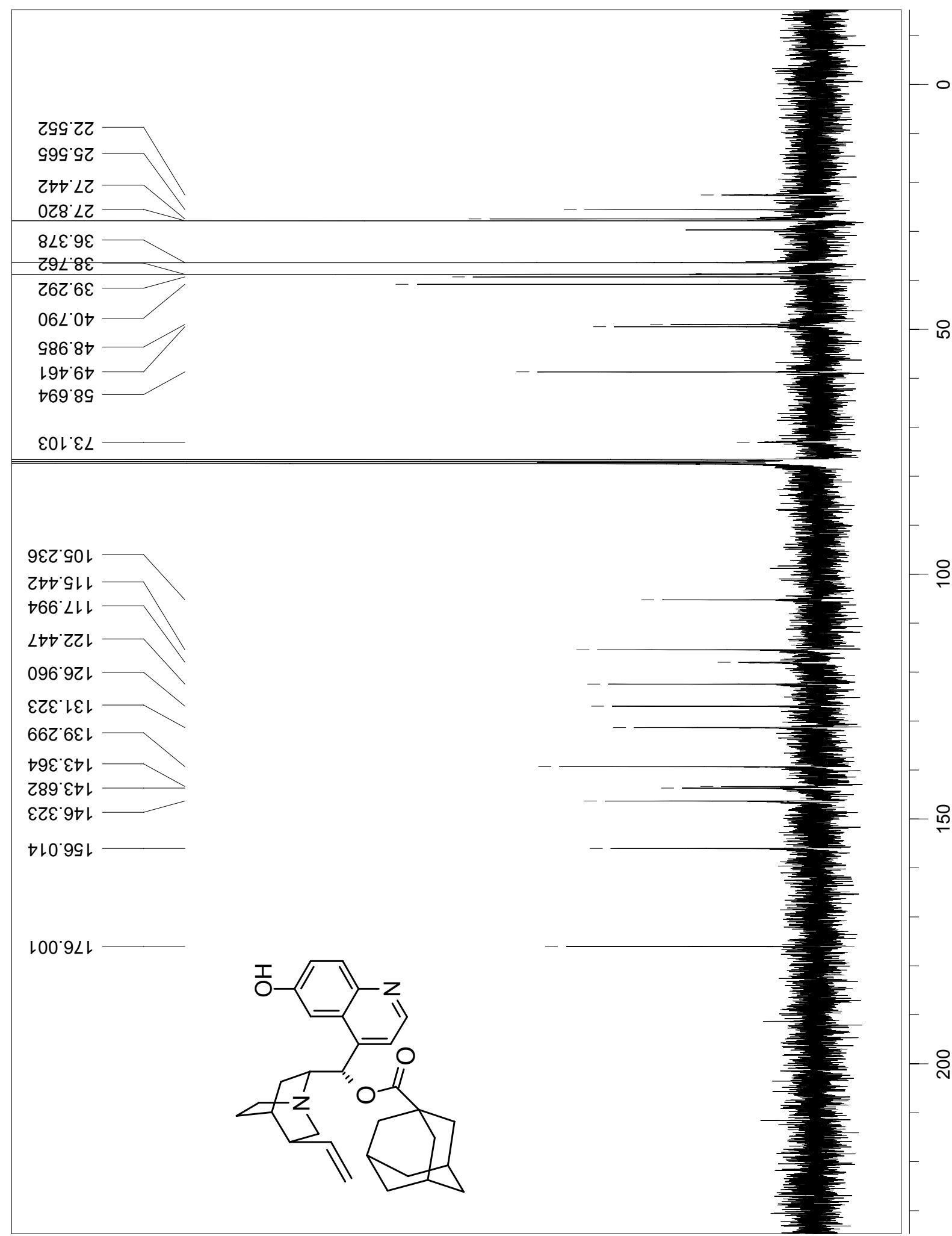

